Supporting Information for

\title{
Base Promoted Three-Component Annulation of 4-Diazoisochroman-3-imines with Dimethylsulfonium Ylides: Synthesis of Highly Functionalized Isochromeno[4,3-c]pyridazines
}

Muhammad Suleman, Jianwei Xie, Zaibin Wang, Ping Lu* and Yanguang Wang* Department of Chemistry, Zhejiang University, Hangzhou, 310027 P. R. China pinglu@zju.edu.cn; orgwyg@zju.edu.cn

\section{Table of Content}

1. X-Ray crystallography of 3a and 4 (Method and instrumentation) ........S1

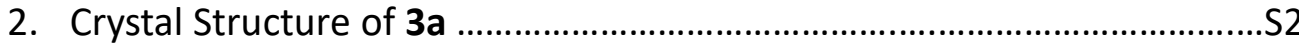

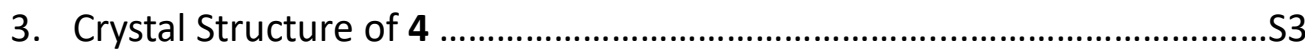

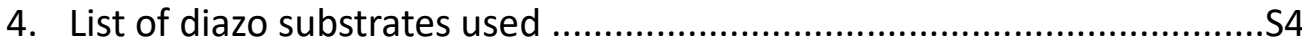

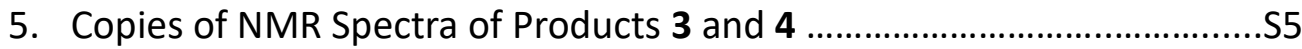




\section{X-Ray Crystallography of 3a and 4}

\section{Method for Crystal growth of 3a and 4}

The solid product $15 \mathrm{mg}$ was added to a $25 \mathrm{~mL}$ round bottom flask fixed with a reflux condenser. One $\mathrm{mL}$ of $n$-hexane was added to the flask and heated to the boiling point of solvent. After the $n$-hexane started to boil and cool down by reflux condenser, one $\mathrm{mL}$ of ethyl acetate (EA) was added to the flask. The solvent system $n$-hexane/EA was added in 1:1 ratio with continues stirring and heating, until all the solid was dissolved. Flask was kept with reflux condenser on for several days at room temperature, until tiny crystals started to grow in the bottom of flask, which subsequently grew with time.

\section{Procedure for crystal measurement of $3 a$ and 4}

A suitable crystal $(0.01 \mathrm{~mm} * 0.02 \mathrm{~mm} * 0.03 \mathrm{~mm})$ was selected and mounted on a Bruker D8 venture diffractometer with Mo Ka radiation $(\lambda=0.71073 \AA)$ for cell determination and subsequent data collection at $170 \mathrm{~K}$. Using Olex2, ${ }^{1}$ the structure was solved with the ShelXT ${ }^{2}$ structure solution program using Intrinsic Phasing and refined with the ShelXL ${ }^{3}$ refinement package using Least Squares minimization.

\section{References}

(1) Dolomanov, O.V.; Bourhis, L. J.; Gildea, R. J.; Howard, J. A. K.; Puschmann, H., OLEX2: a complete structure solution, refinement and analysis program, J. Appl. Cryst. 2009, 42, 339-341.

(2) Sheldrick, G. M., SHELXT - Integrated space-group and crystal structure determination, Acta Cryst. 2015, A71, 3-8.

(3) Sheldrick, G. M. Crystal structure refinement with SHELXL, Acta Cryst. 2015, C71, 3-8. 
The ORTEP and Crystal parameters of $3 \mathrm{a}$ wherein thermal ellipsoid are drawn at $\mathbf{3 0 \%}$ probability (CCDC 2013407)

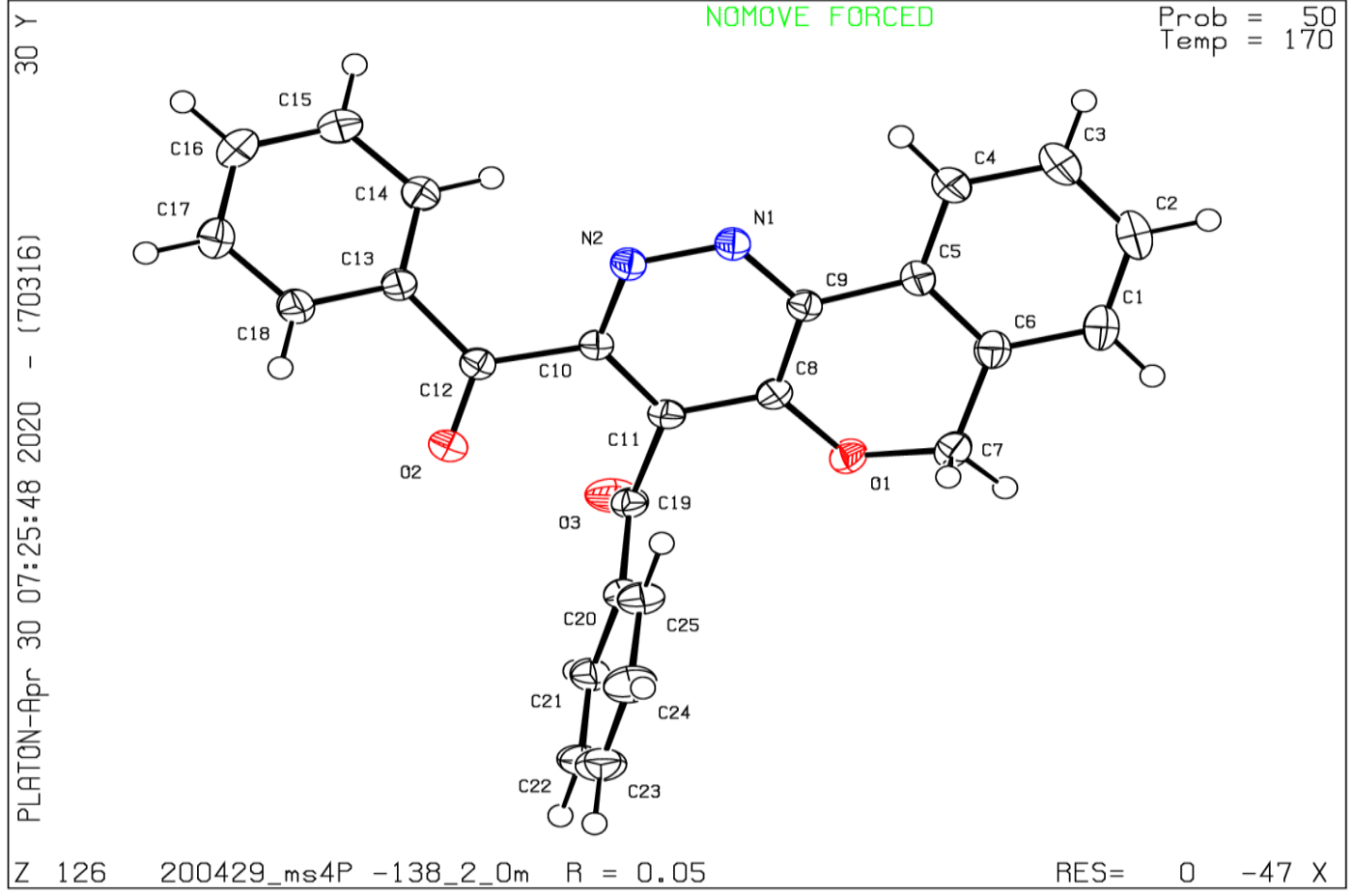

Datablock: 200429_ms438_138_2_0m

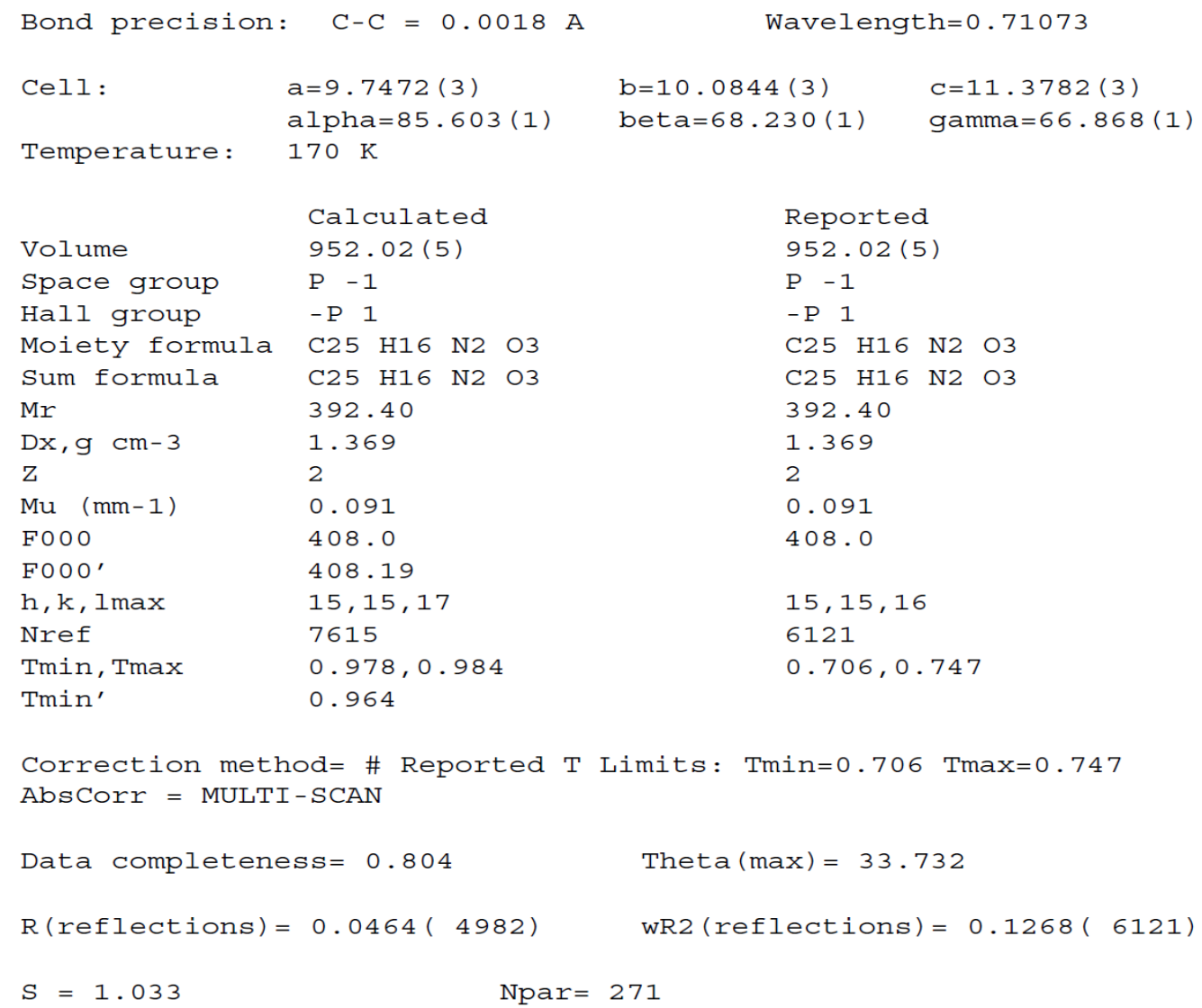

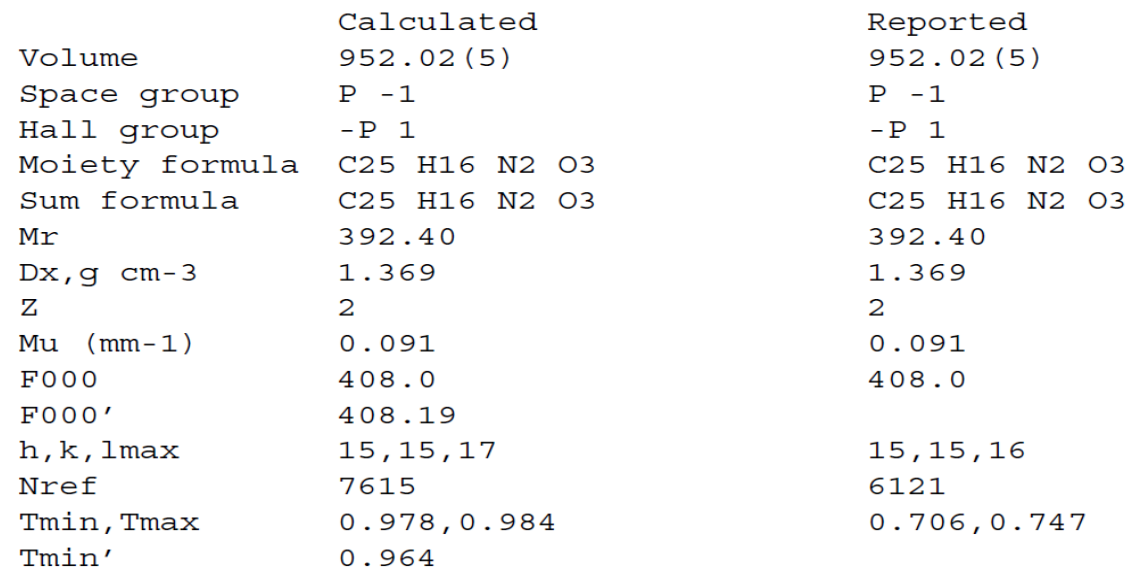

Correction method= \# Reported T Limits: Tmin=0.706 Tmax=0.747 AbsCorr = MULTI-SCAN 
The ORTEP and Crystal parameters of 4 wherein thermal ellipsoid are drawn at $30 \%$ probability (CCDC 2013408)

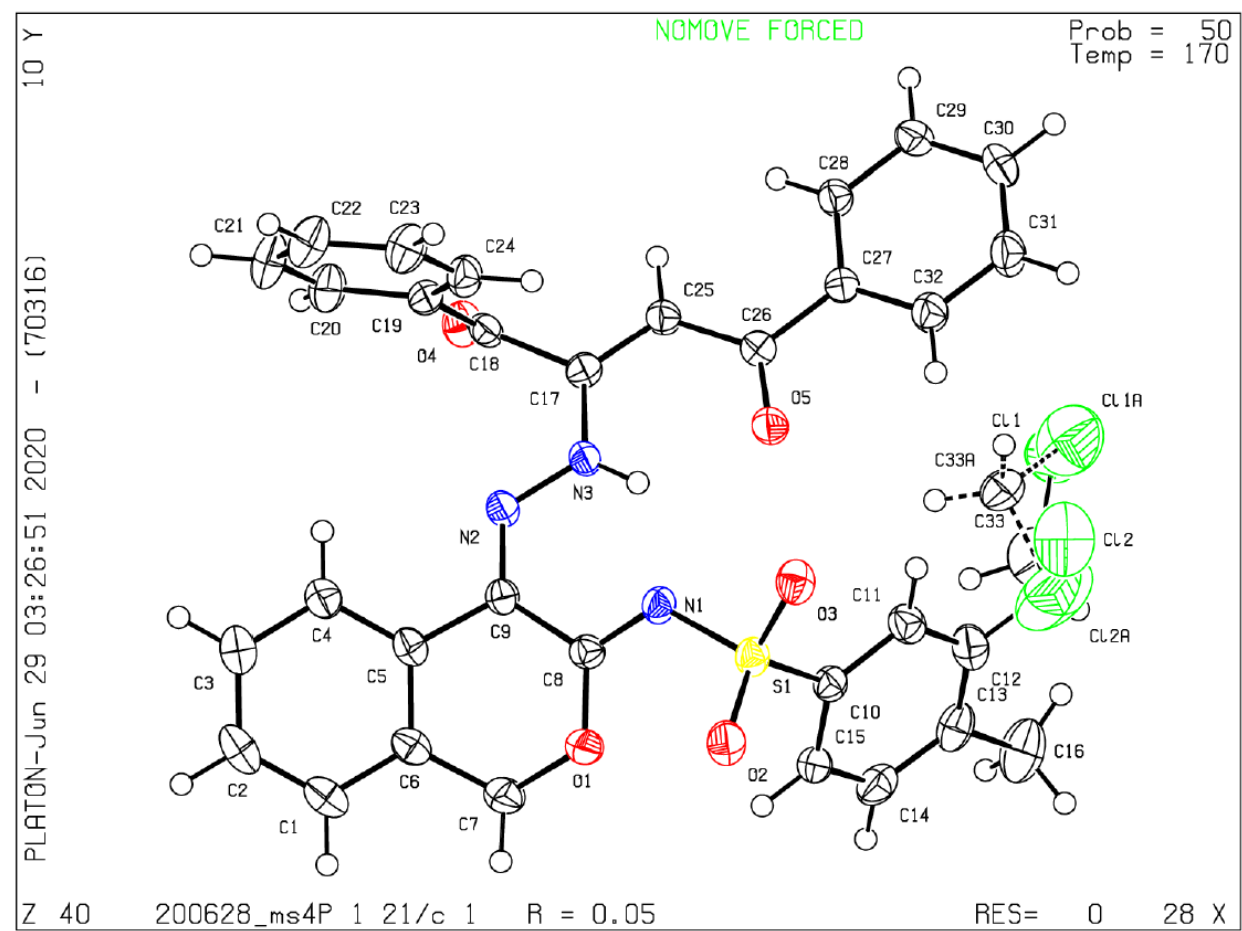

\section{Datablock: 200628_ms438_93_4_0m}

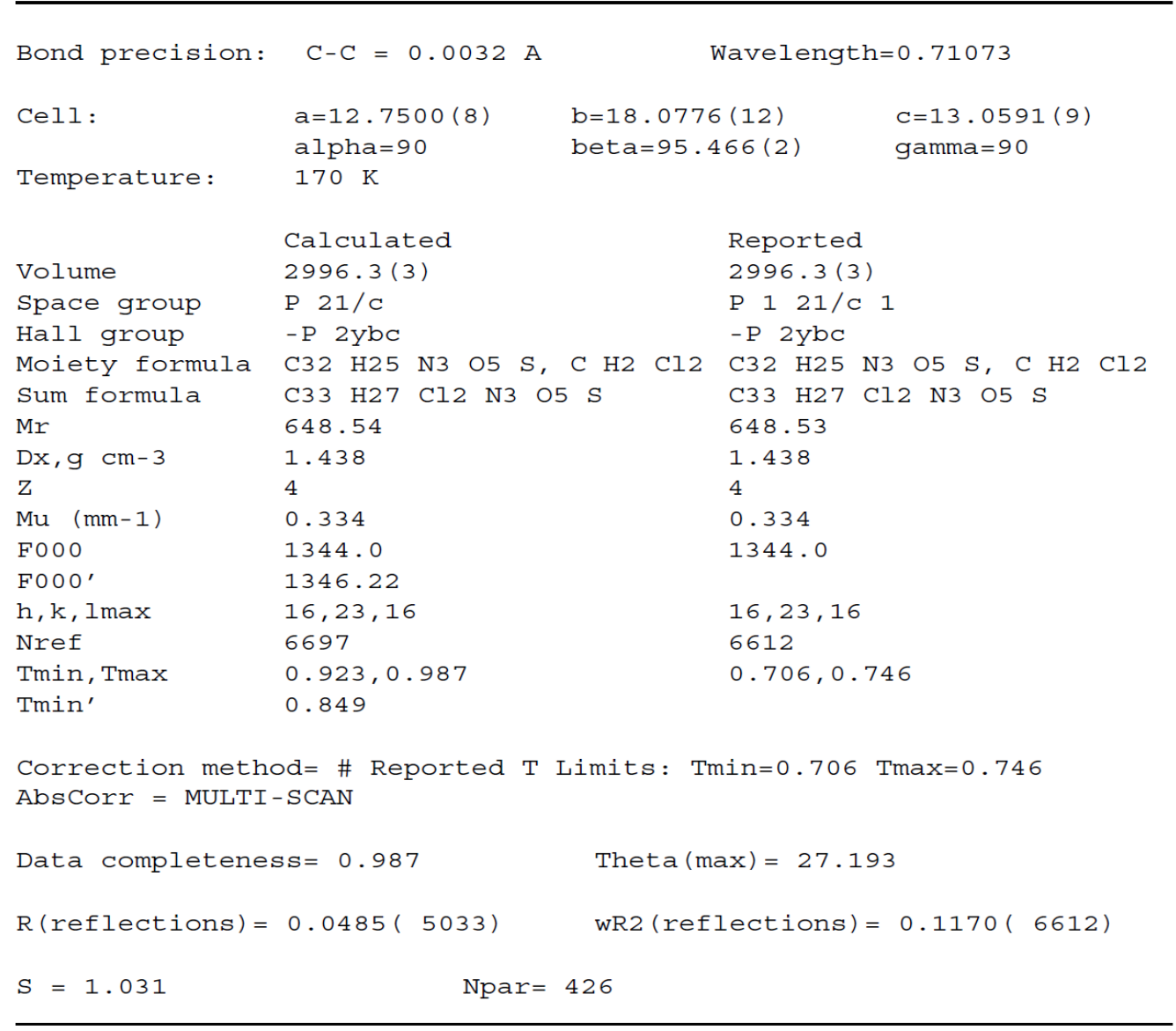


List of Diazo substrates used: All these diazo compounds are known and were synthesized according to our published procedure. ${ }^{1}$<smiles>[N-]=[N+]=C1C(=[N-])OCc2ccccc21</smiles>

$1 \mathrm{a}$<smiles>[N-]=C1OCc2cc(C(F)(F)F)ccc2C1=[NH2+]</smiles>

$1 \mathrm{~b}$<smiles>[N-]=[N+]=C1OCc2cc(F)ccc2C1=[N+]=[N-]</smiles>

$1 \mathrm{c}$<smiles>[Y15]=C1OCc2cc(Cl)ccc2C1=[NH2+]</smiles>

1d<smiles>COc1ccc2c(c1)COC(=[N+]=[N-])C2=[N+]=[N-]</smiles><smiles>[N+]=C1COCc2ccc(F)cc2C1=[N+]=[N-]</smiles><smiles>[NH2+]=C1OCc2ccc(Cl)cc2C1=[NH2+]</smiles><smiles>COc1ccc2c(c1)C(=N)C(=[N+]=[N-])OC2</smiles><smiles>CC1OC(=[N+]=[N-])C(=[W])c2ccccc21</smiles><smiles>[N-]=[N+]=C1OC(c2ccccc2)c2ccccc2C1=N</smiles><smiles>[N-]=[N+]=C1OCc2cc3c(cc2C1=[NH2+])OCO3</smiles><smiles>C/C=C\C=C/C=N/S(=O)(=O)c1ccc(Cl)cc1</smiles><smiles>N=C1C(=NS(=O)(=O)c2ccccc2)OCc2ccccc21</smiles><smiles>C/C=C\CC1OCc2ccccc2C1=NO</smiles>
11<smiles>C/C=C\C</smiles><smiles>N=C1/C(=N\S(=O)(=O)c2ccc3ccccc3c2)OCc2ccccc21</smiles>
$1 \mathrm{n}$<smiles>CS(=O)(=O)/N=C1/OCc2ccccc2C1=N</smiles>

$1 p$

List of Sulfonium Salts used: All sulfonium salts are reported compounds and were synthesized according to a published procedure. ${ }^{2}$<smiles>C[Sb](Br)CC(=O)c1ccccc1</smiles><smiles>COc1ccccc1C(=O)C[S+](C)Br</smiles><smiles>Cc1ccccc1C(=O)C[S+](C)Br</smiles><smiles>Cc1cccc(C(=O)C[S+](C)C)c1</smiles><smiles>COc1ccc(C(=O)C[Sn](C)C[Sn](C)C[Sn](C)CC(=O)c2cccc(Cl)c2)cc1</smiles><smiles>Cc1ccc(C(=O)C[Sn](C)CC(=O)c2ccc(F)cc2)cc1</smiles><smiles>C[Sb](C)CC(=O)c1ccc(Cl)cc1</smiles><smiles>C[S+](C)CC(=O)c1ccc(C(=O)C[Sn](C)C)cc1</smiles><smiles>C[S+](C)CC(=O)c1ccc([Se][S+](C)(C)CC(=O)c2ccc3ccccc3c2)s1</smiles>

\section{References}

1. Ren, A. N.; Lu, P.; Wang, Y. G., Convenient preparation of 4-diazoisochroman-3-imines and 3subsituted 3,5-dihydroisochromeno-[3,4-d] [1,2,3] triazoles. Chem. Commun. 2017, 53, 3769-3772.

2. Zhang, X. Z.; Du, J. Y.; Deng, Y. H.; Chu, W. D.; Yan, X.; Yu, K. Y.; Fan, C. A., Spirocyclopropanation Reaction of para-Quinone Methides with Sulfonium Salts: The Synthesis of Spirocyclopropanyl para-Dienones. J. Org. Chem.2016, 81, 2598-2606. 


\section{NMR Spectra of Products 3 and 4}

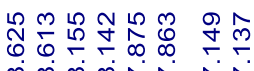

$\infty \infty \infty \infty i \pi$

워
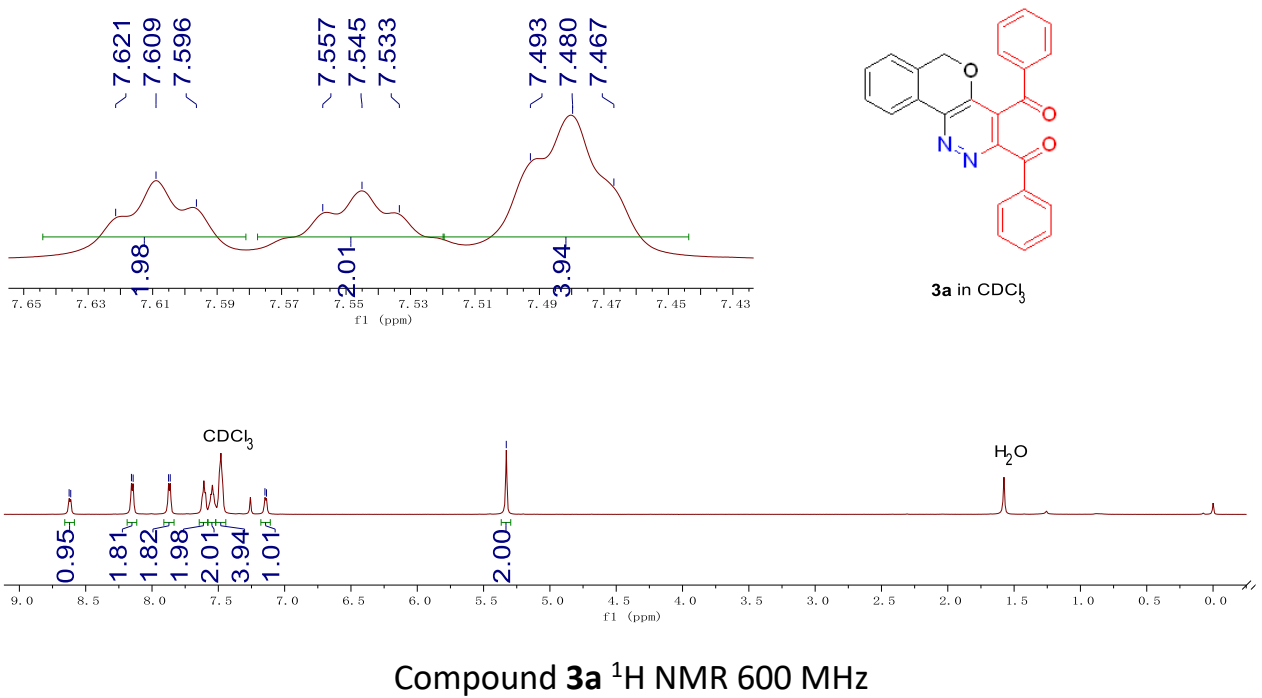

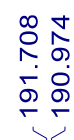

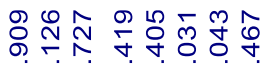

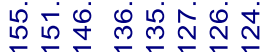

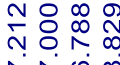

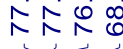

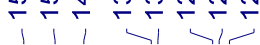

$\mathrm{CDC}_{3}$
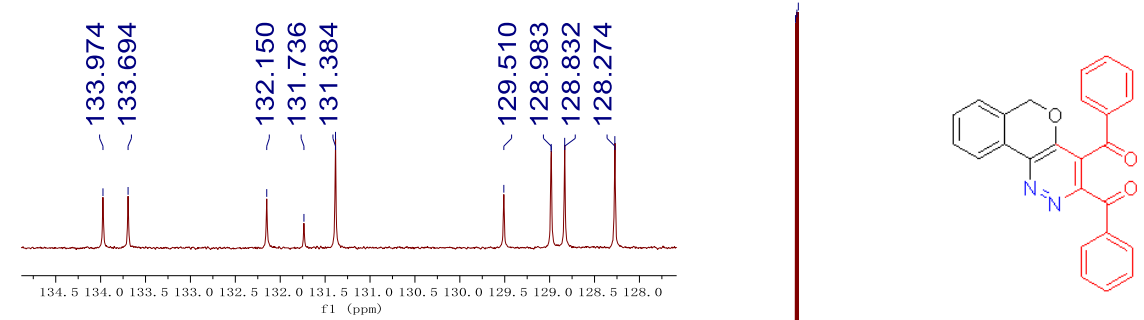

3a in $\mathrm{CDCl}_{3}$
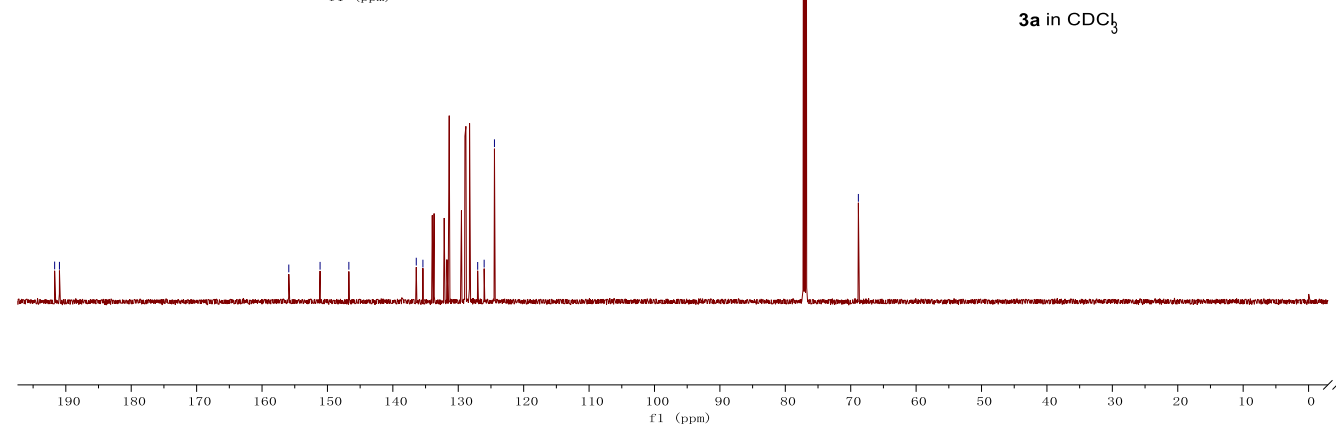

Compound $3 a{ }^{13} \mathrm{C}$ NMR $150 \mathrm{MHz}$ 


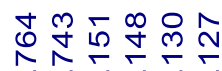

$\infty \infty \infty \infty \infty \infty$

ి

$\infty$
$\infty$
0
0

$\longrightarrow \longrightarrow$
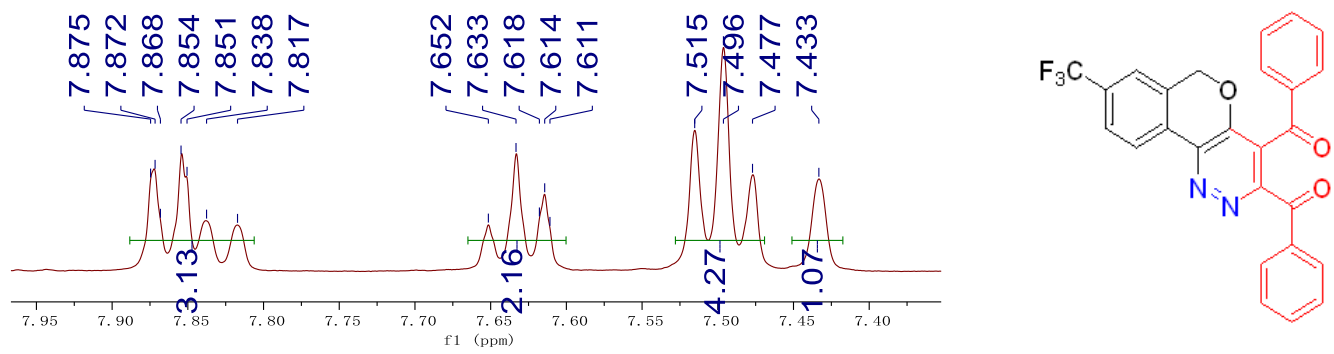

$\mathbf{3 b}$ in $\mathrm{CDCb}_{3}$

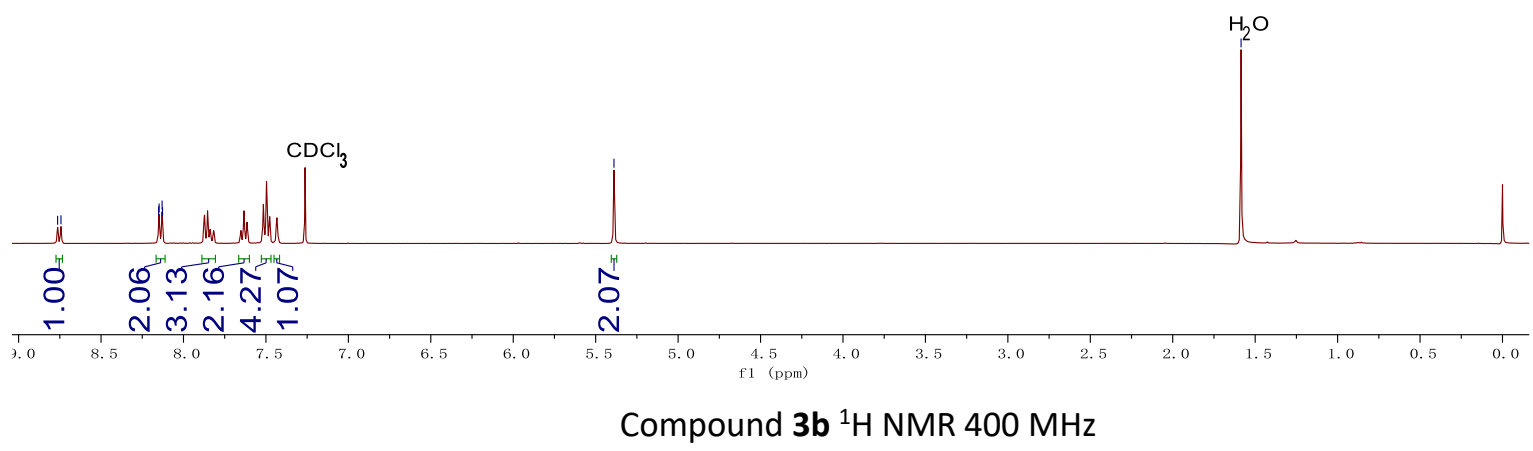

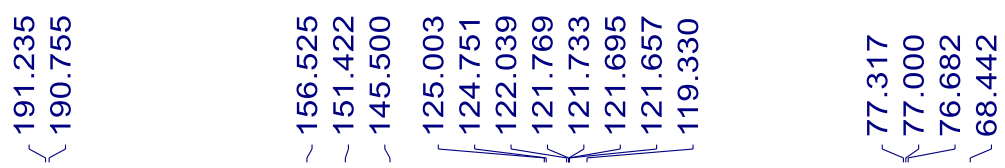

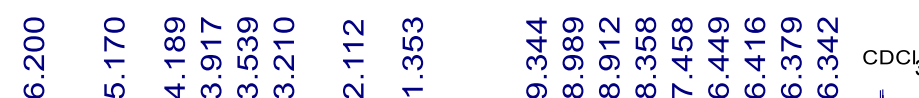

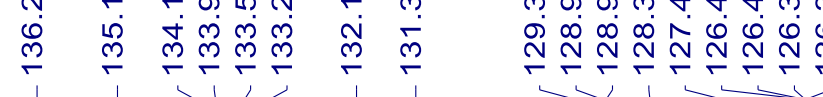
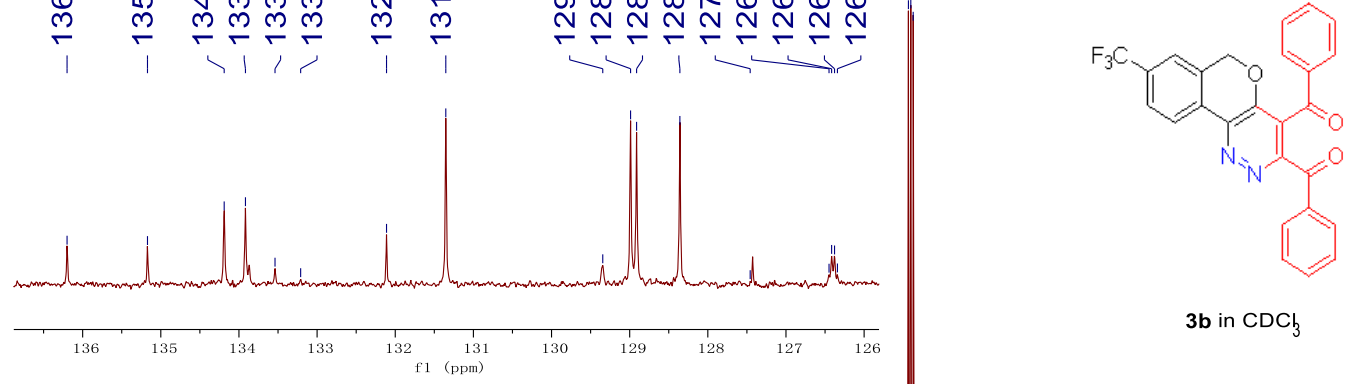

$\mathbf{3 b}$ in $\mathrm{CDCl}_{3}$

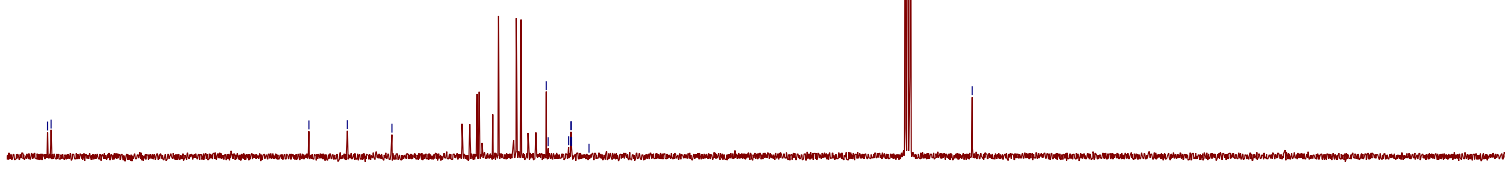

Compound $3 b{ }^{13} \mathrm{C}$ NMR $100 \mathrm{MHz}$ 


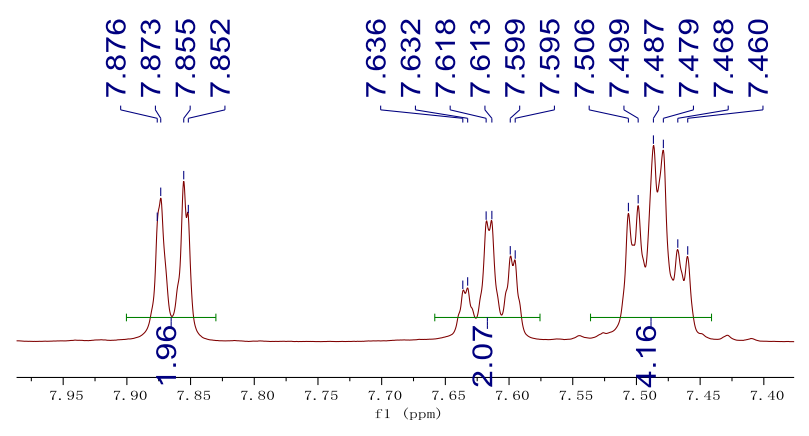

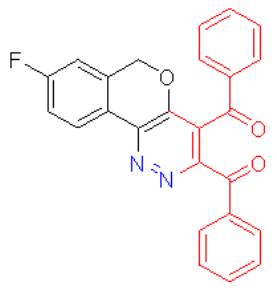

$3 \mathrm{c}$ in $\mathrm{CDCl}_{3}$

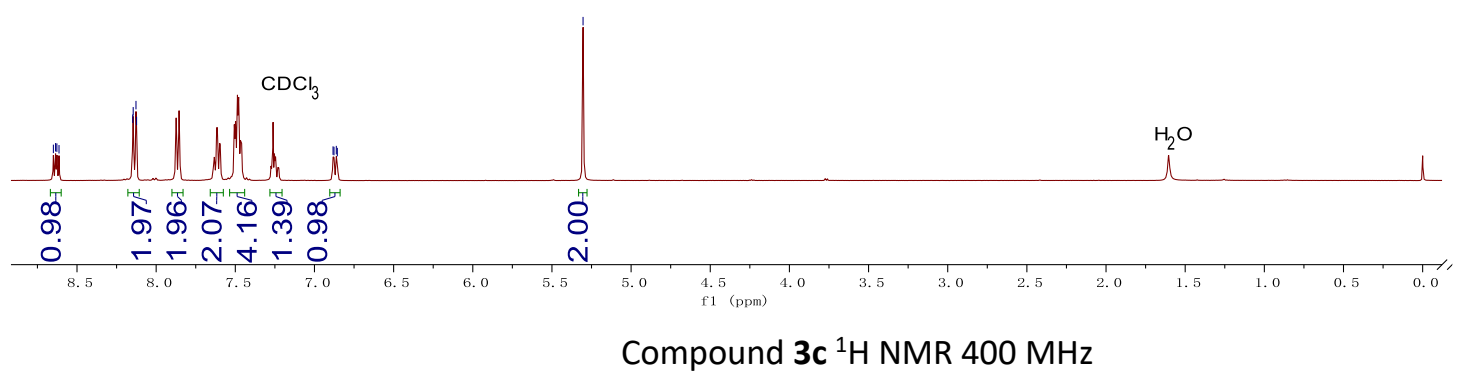

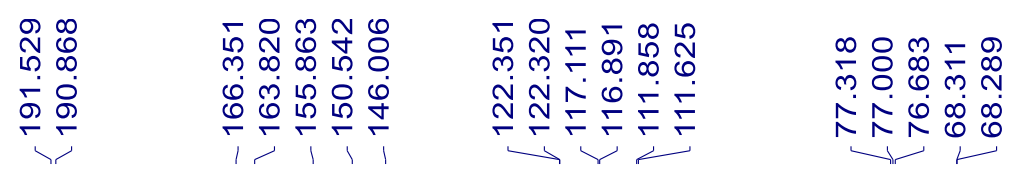
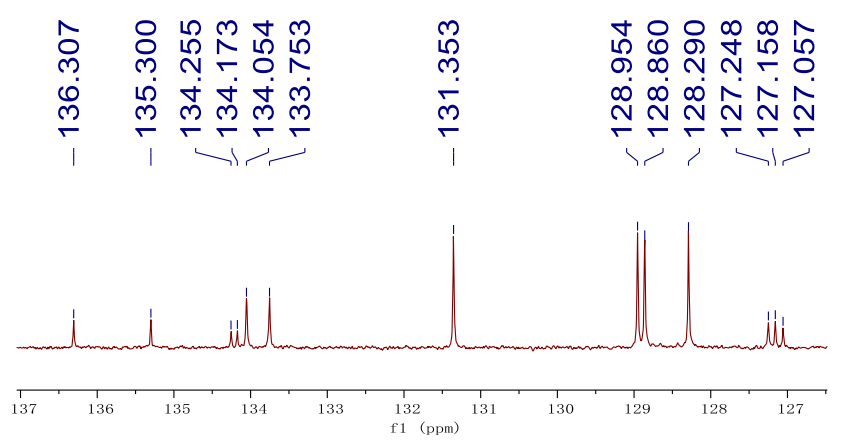

$\mathrm{CDCl}_{3}$

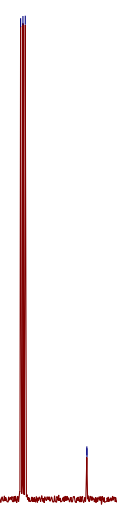

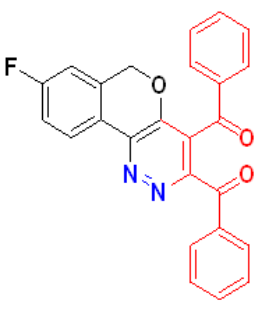

$3 \mathrm{c}$ in $\mathrm{CDCl}_{3}$

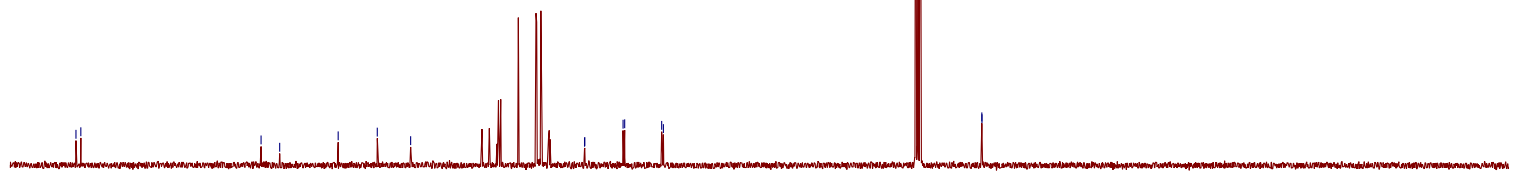

Compound 3c ${ }^{13} \mathrm{C}$ NMR $100 \mathrm{MHz}$ 


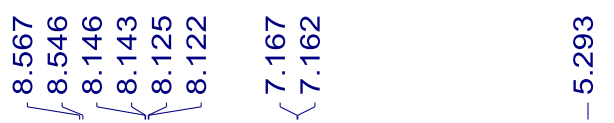

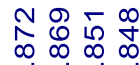

Nin

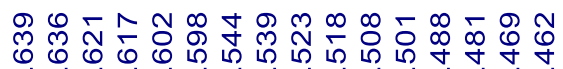

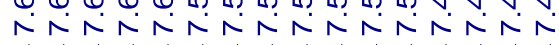

$\rightarrow 10$

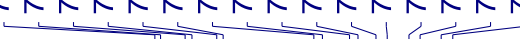
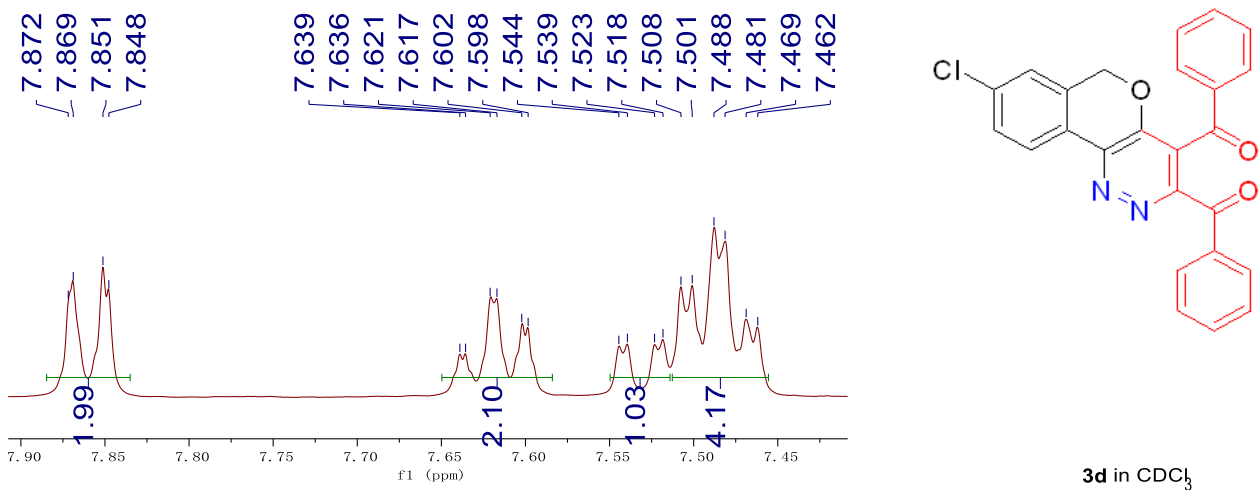

3d in $\mathrm{CDC}$

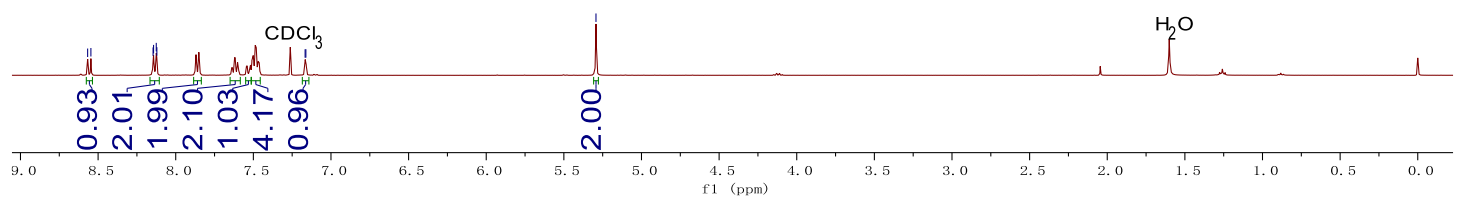

Compound 3d ${ }^{1} \mathrm{H}$ NMR $400 \mathrm{MHz}$

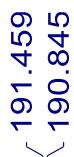

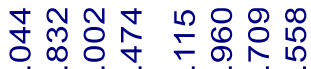

要 $\quad \frac{i}{i}$

동

ヘペ䒘

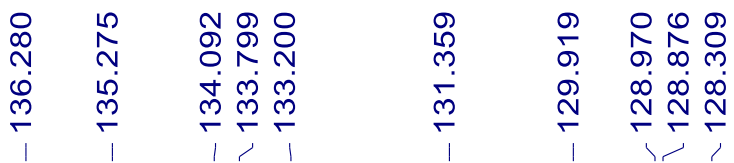
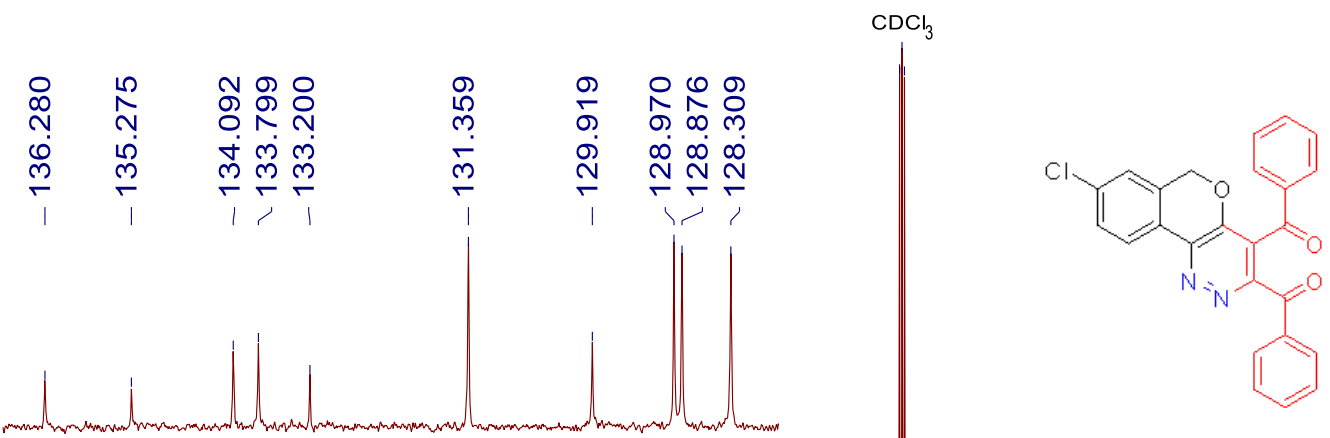

3d in $\mathrm{CDCl}_{3}$
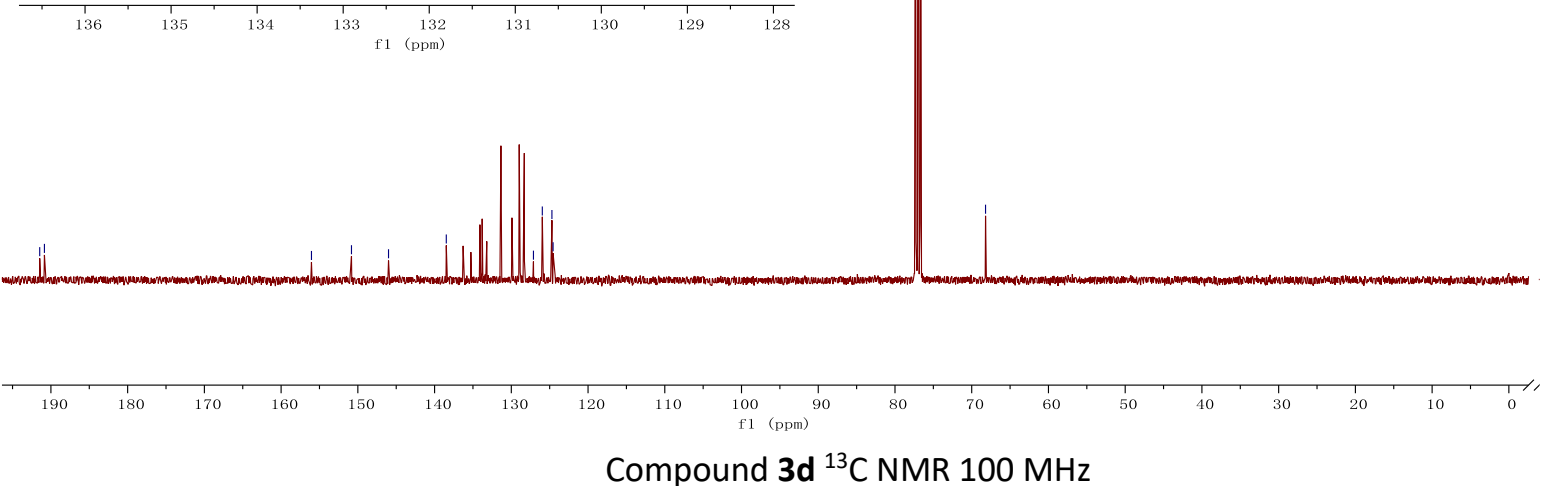


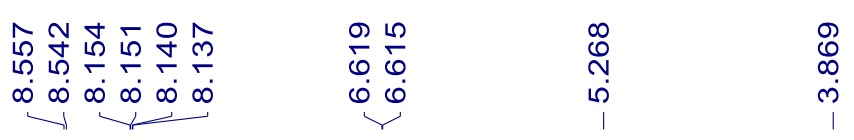

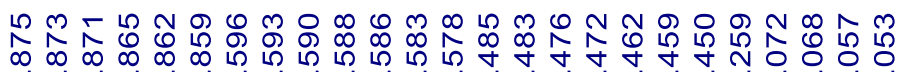

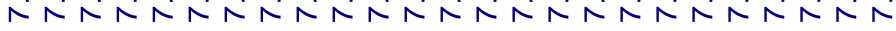

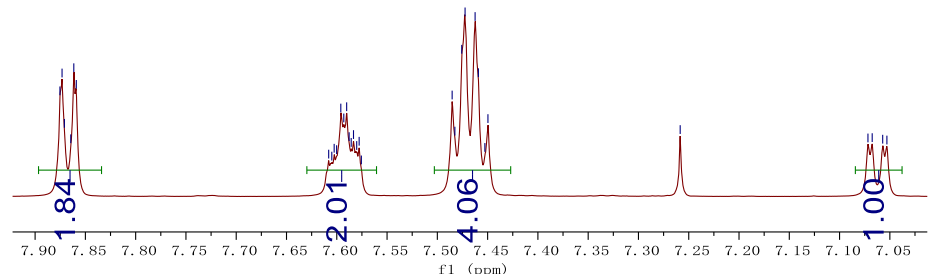

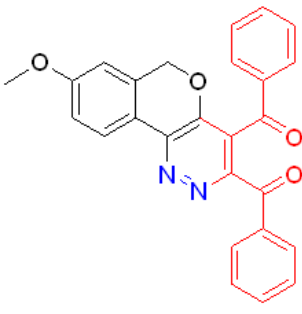

$3 \mathbf{e}$ in $\mathrm{CDCl}_{3}$

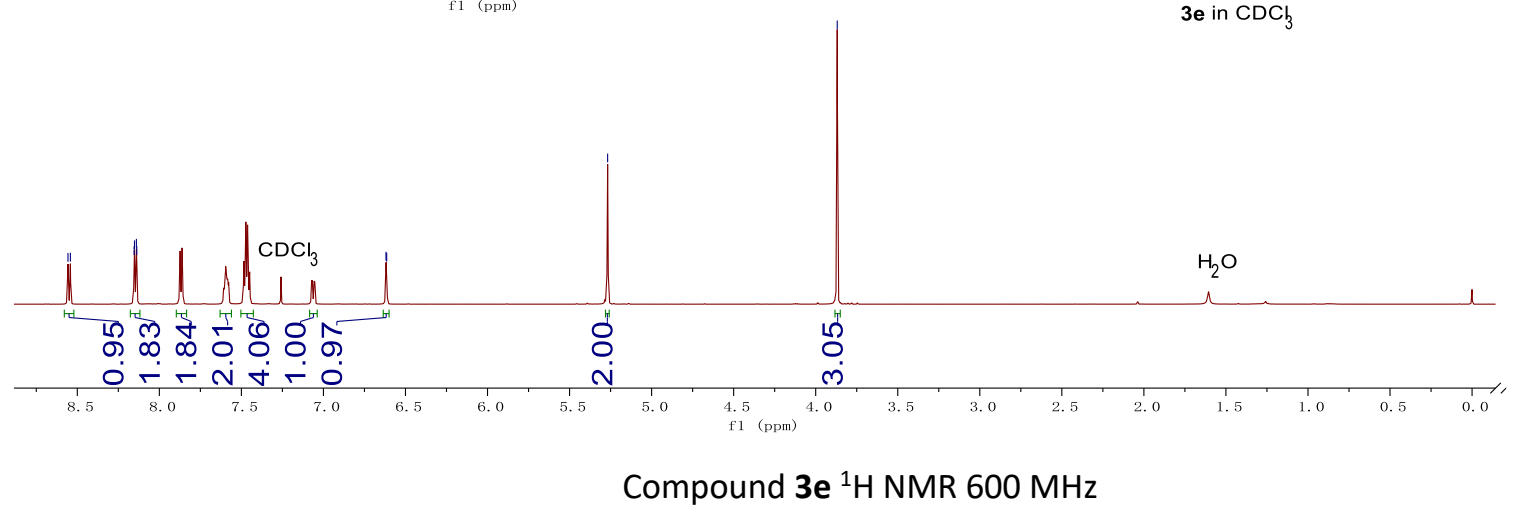

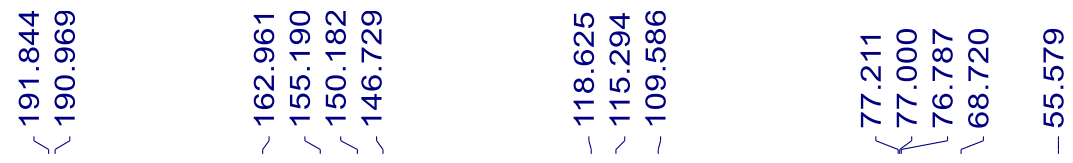
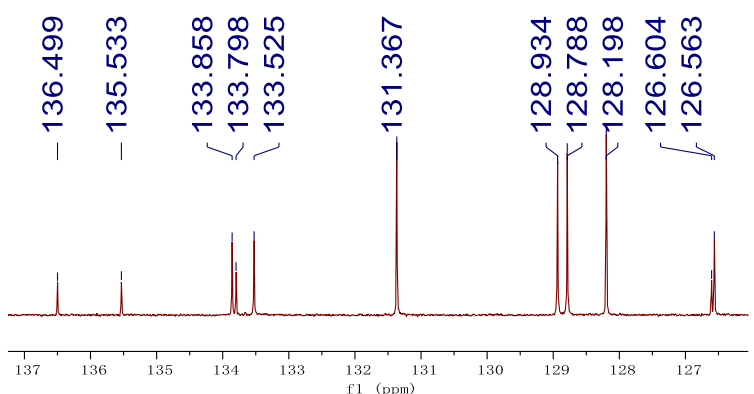

$\mathrm{CDCl}_{3}$

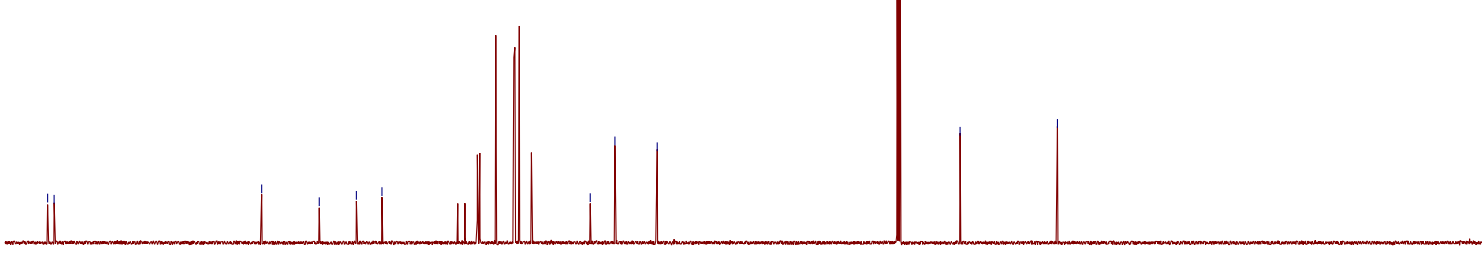

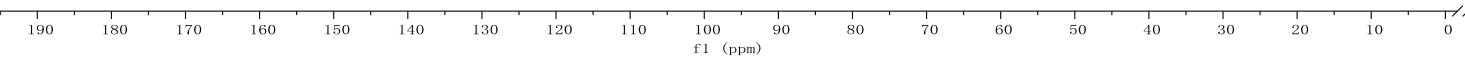

Compound $3 \mathrm{e}^{13} \mathrm{C}$ NMR $150 \mathrm{MHz}$ 
ᄂ)

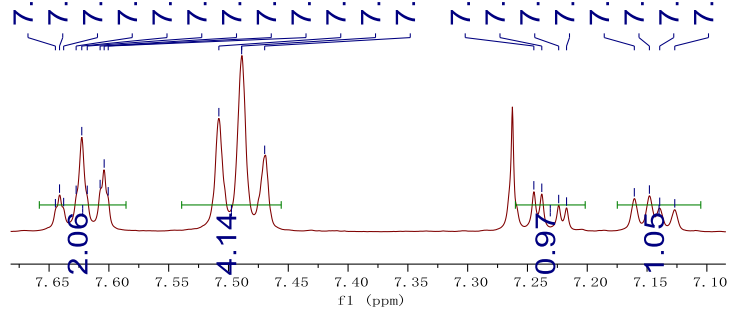

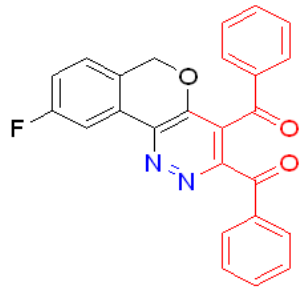

3f in $\mathrm{CDCl}_{3}$

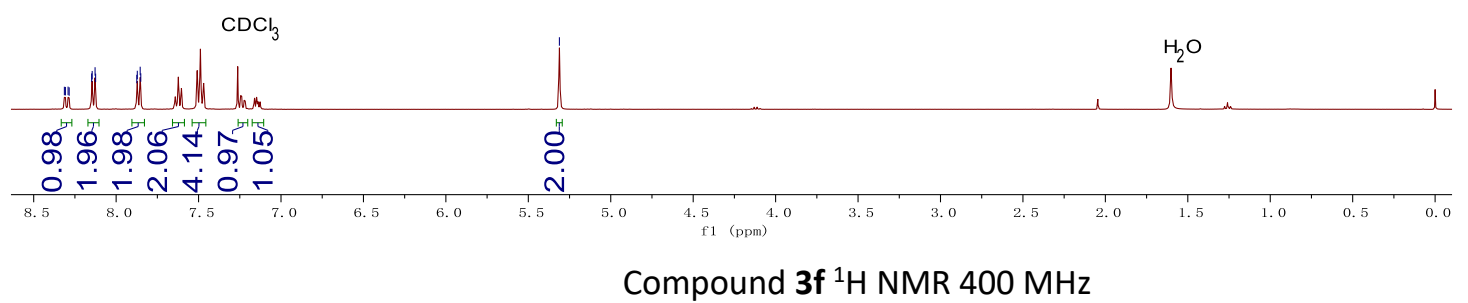

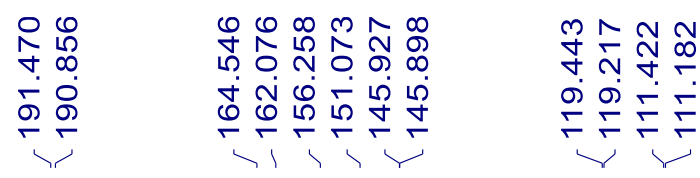

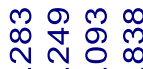

ڤ̆

倠

$\stackrel{\infty}{\sim} \stackrel{\infty}{\sim} \stackrel{\infty}{\sim} \stackrel{\infty}{\sim} \stackrel{\infty}{\sim} \stackrel{\infty}{\sim} \stackrel{\sim}{N} \stackrel{N}{\sim} \stackrel{N}{\sim} \stackrel{i}{\sim} \stackrel{i}{\sim}$
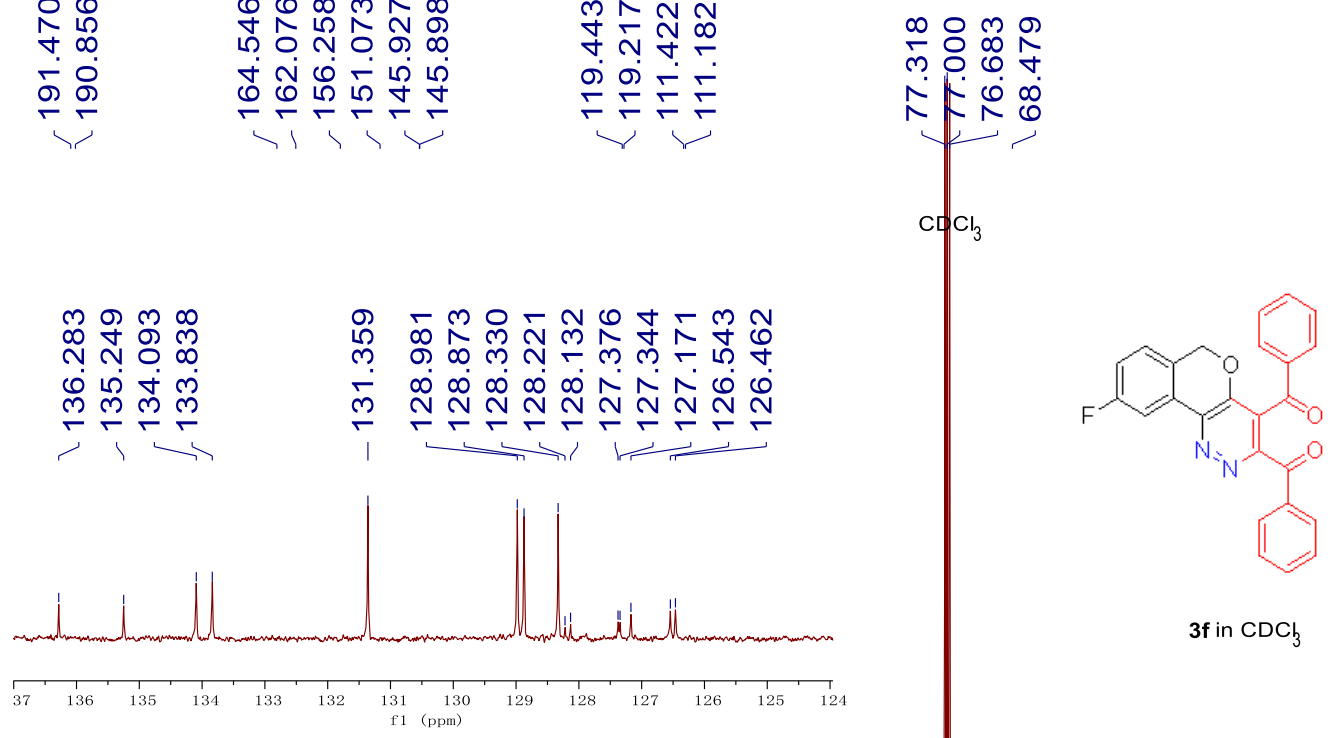

3f in $\mathrm{CDC}_{3}$
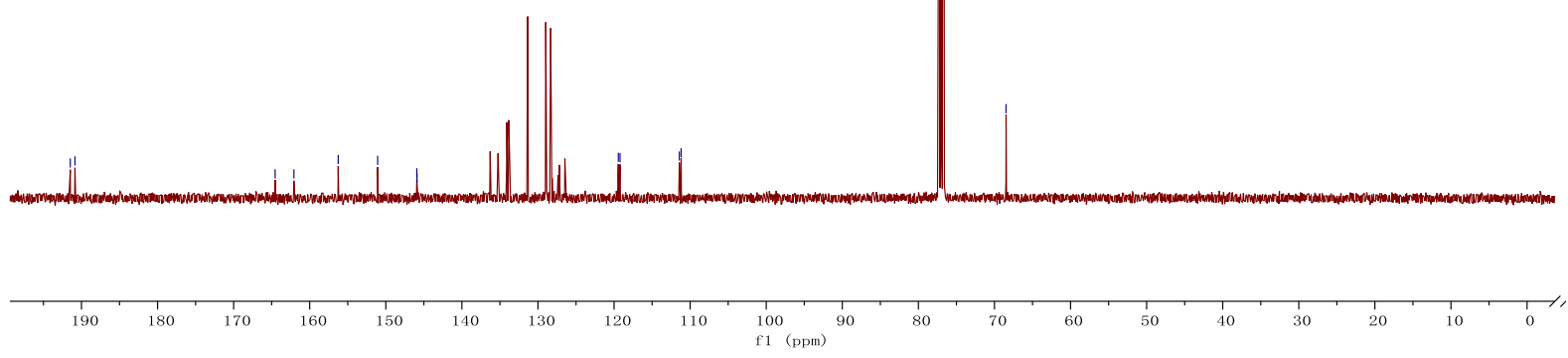

Compound $3 f{ }^{13} \mathrm{C}$ NMR $100 \mathrm{MHz}$ 


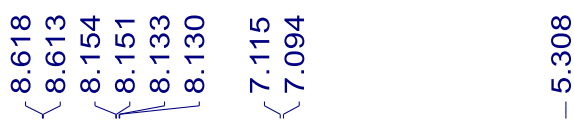

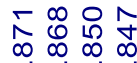

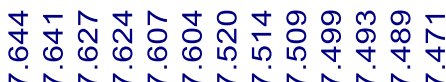

$\wedge N \wedge N$

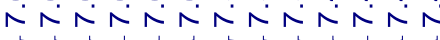

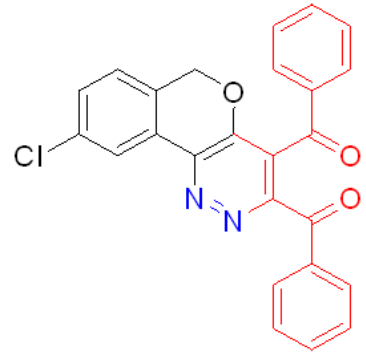

$\mathbf{3 g}$ in $\mathrm{CDCl}_{3}$
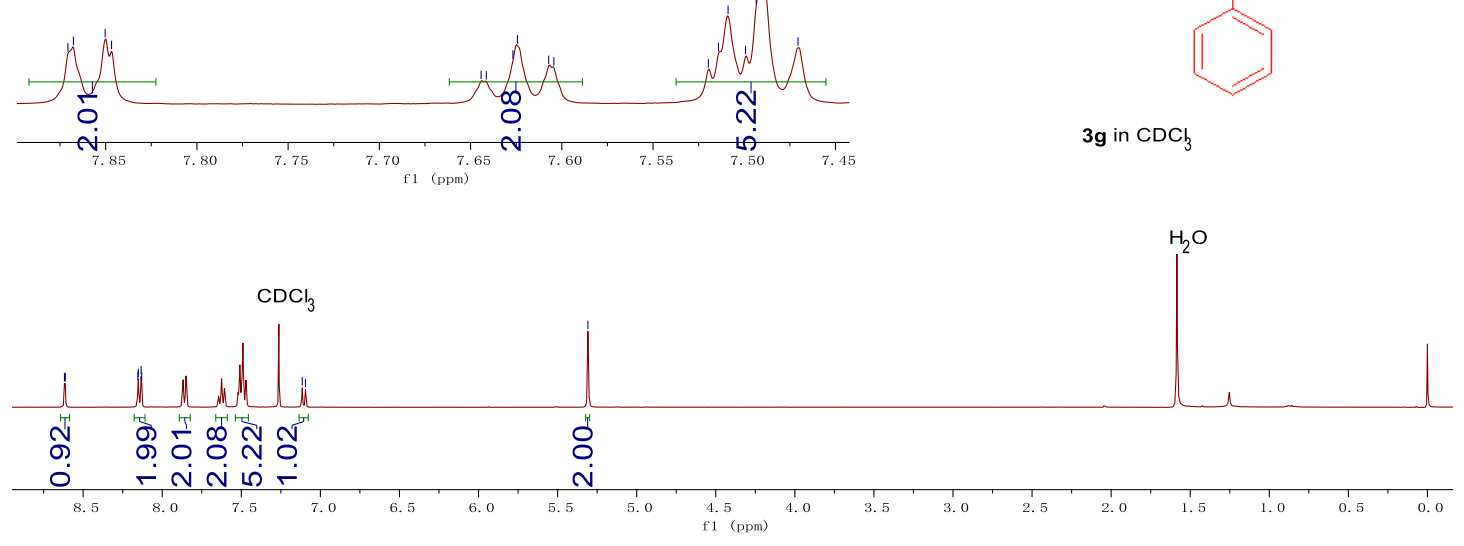

Compound $3 \mathrm{~g}{ }^{1} \mathrm{H}$ NMR $400 \mathrm{MHz}$

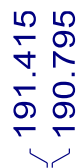

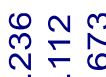

它

๑̊ ₹

กั่

๙ิ

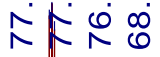

స̦

官
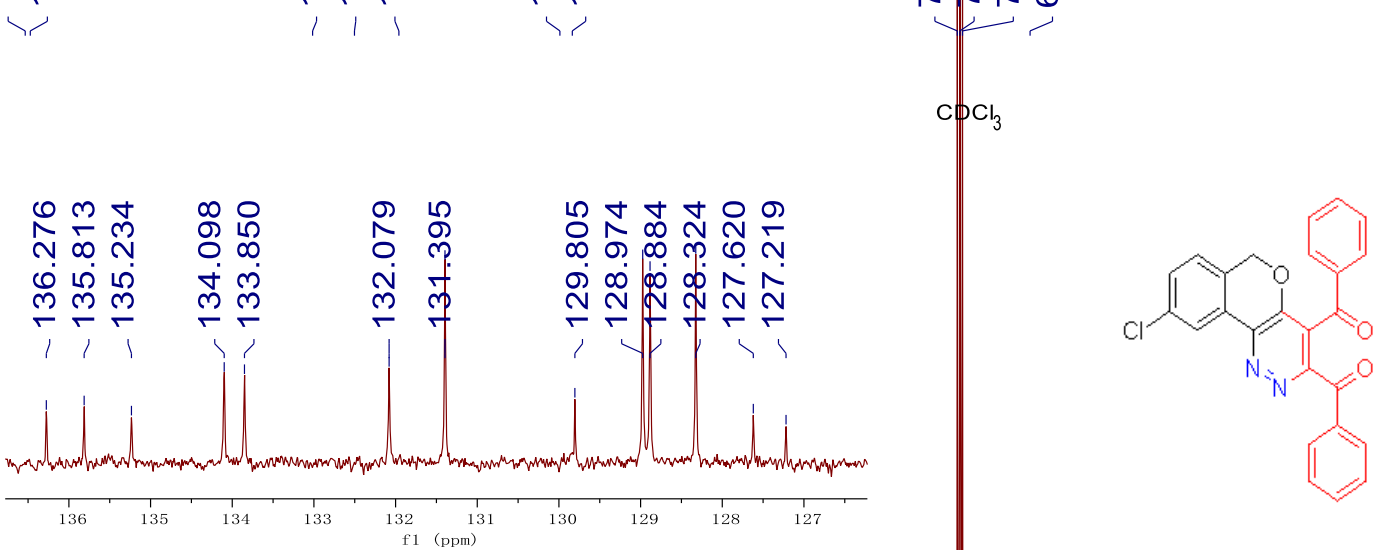

$\mathbf{3 g}$ in $\mathrm{CDCh}$
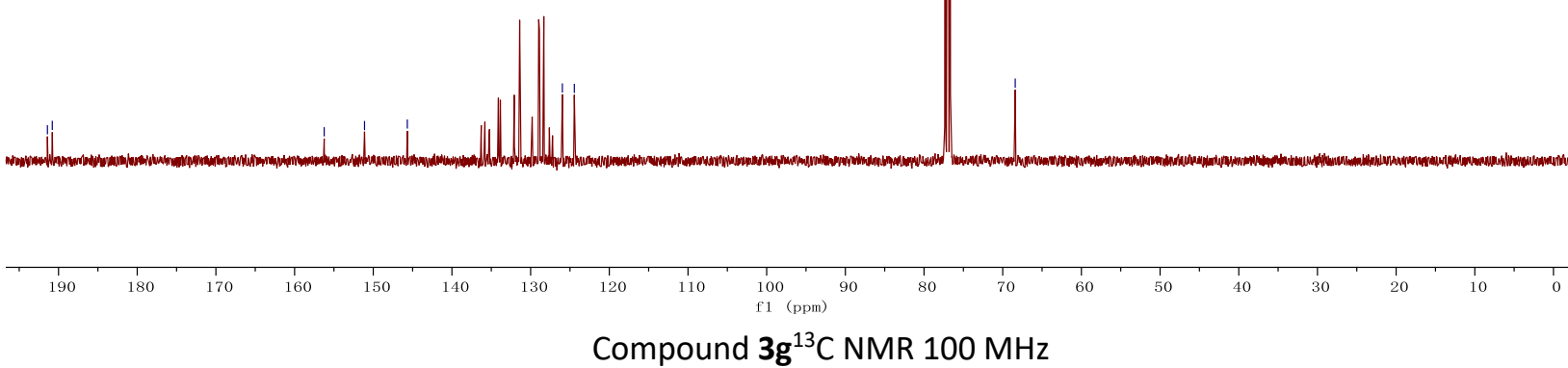

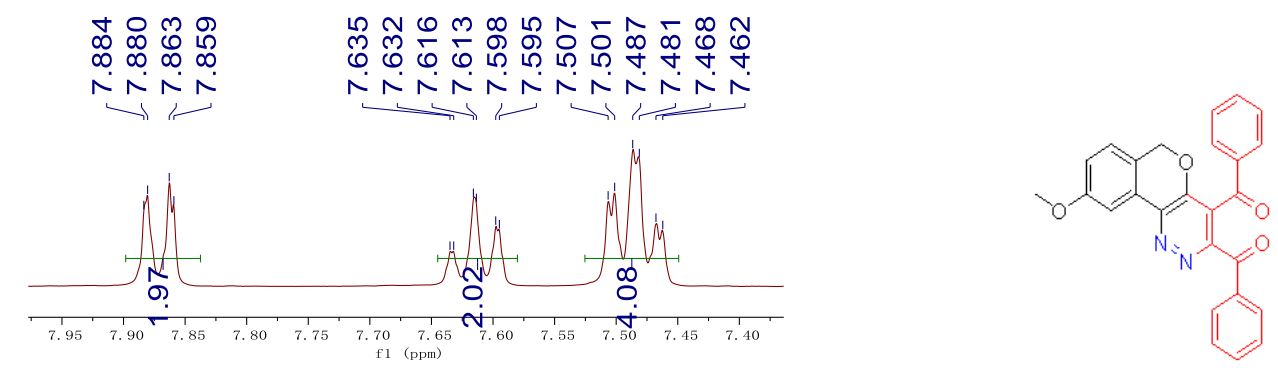

$\mathbf{3 h}$ in $\mathrm{CDCl}_{3}$

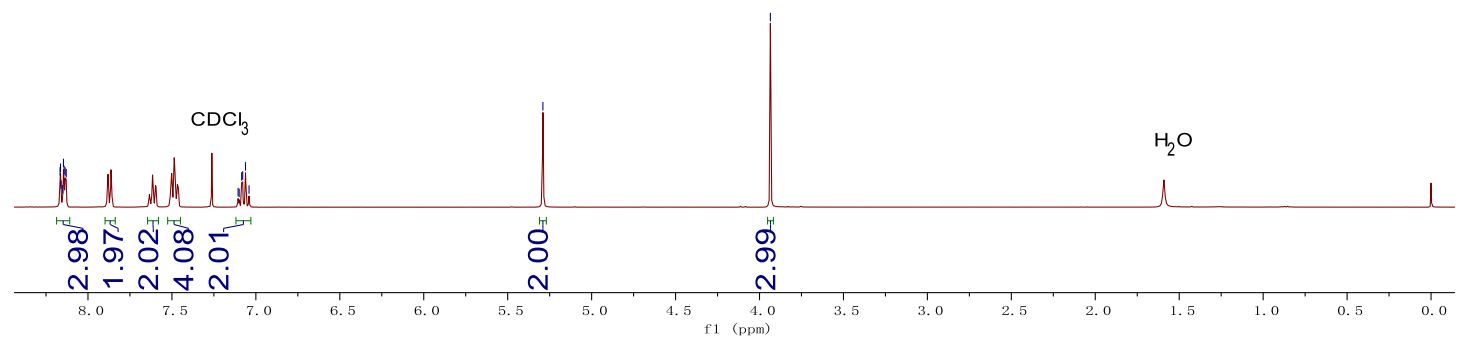

Compound $3 h^{1}{ }^{1} \mathrm{~N}$ NMR $400 \mathrm{MHz}$

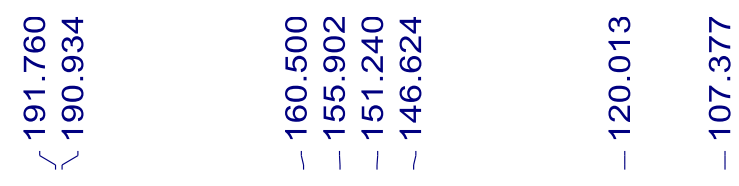

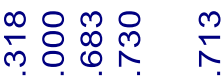

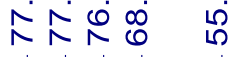

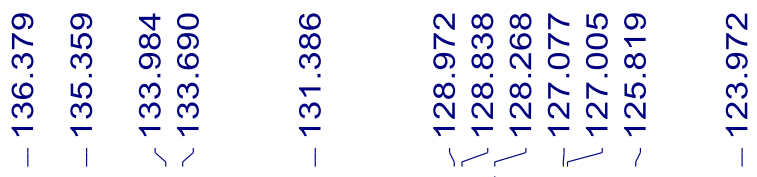
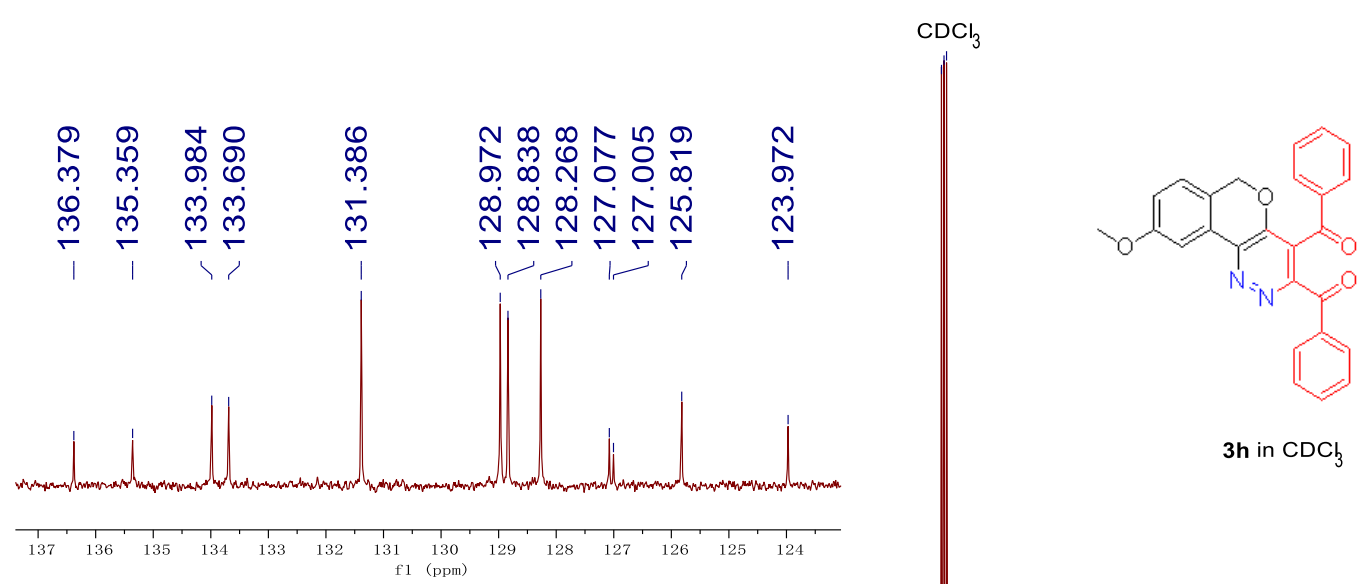

3h in $\mathrm{CDCl}_{3}$
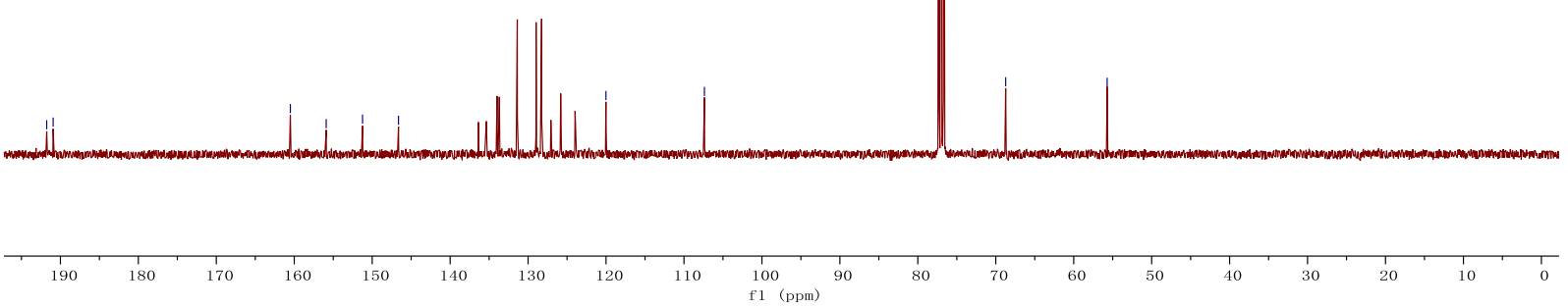

Compound $3 \mathrm{~h}{ }^{13} \mathrm{C}$ NMR $100 \mathrm{MHz}$ 


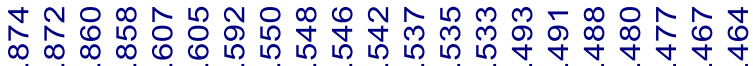

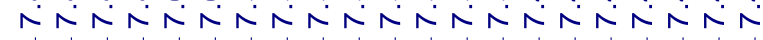
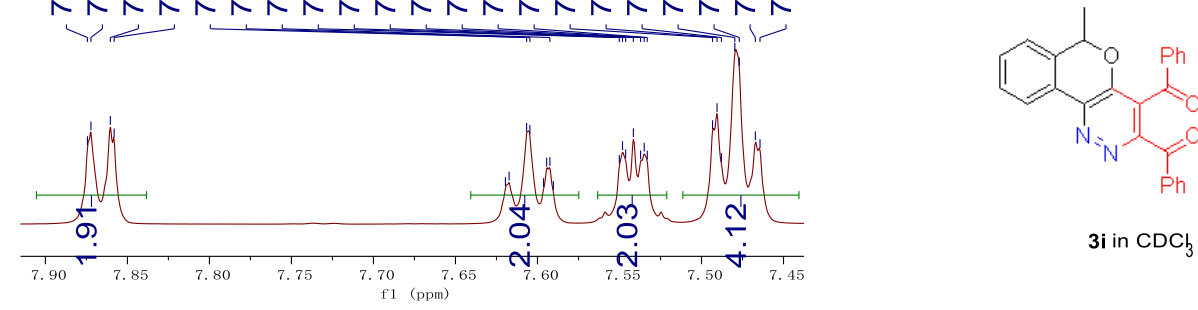

$\mathbf{3 i}$ in $\mathrm{CDCl}_{3}$

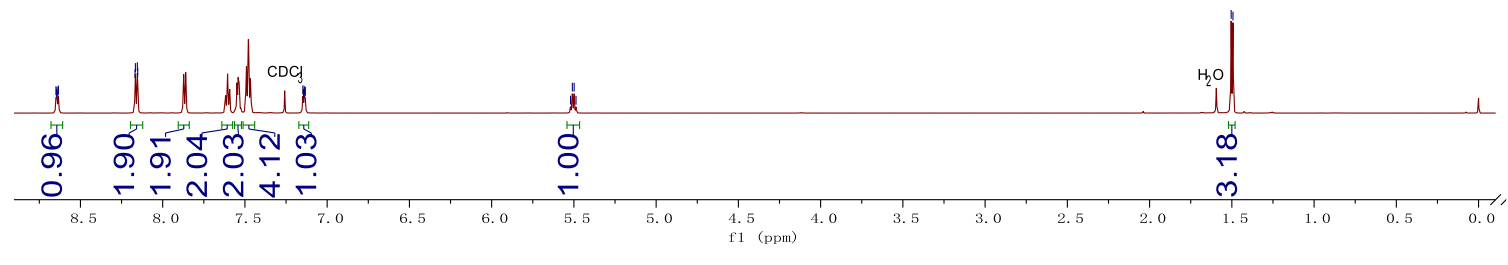

Compound $3^{1}{ }^{1} \mathrm{H}$ NMR $600 \mathrm{MHz}$

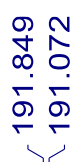

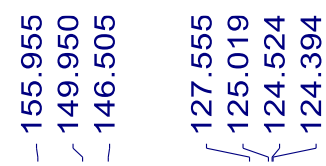

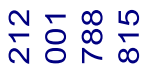

圭定

$\stackrel{0}{\circ}$

$\sin$

กิ่

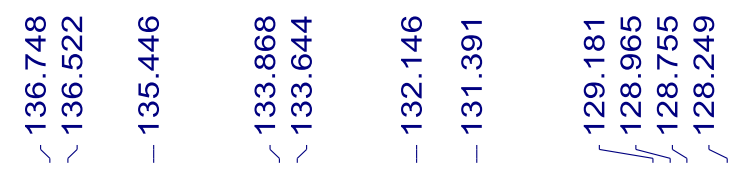
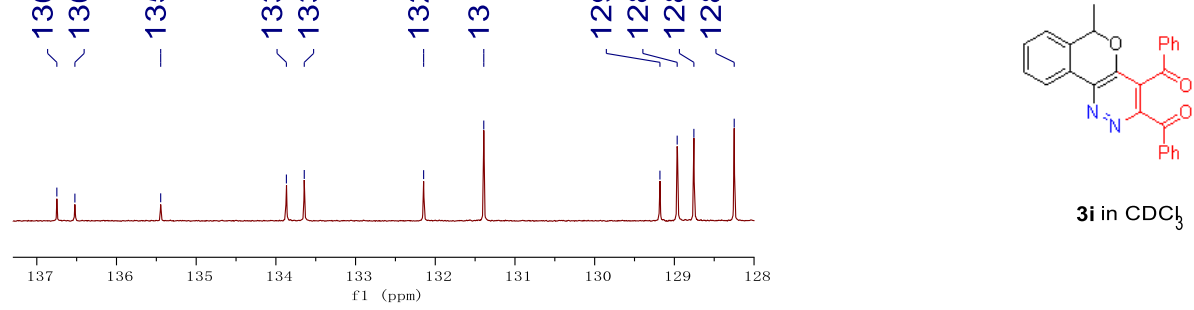

$\mathbf{3 i}$ in $\mathrm{CDC}_{3}$
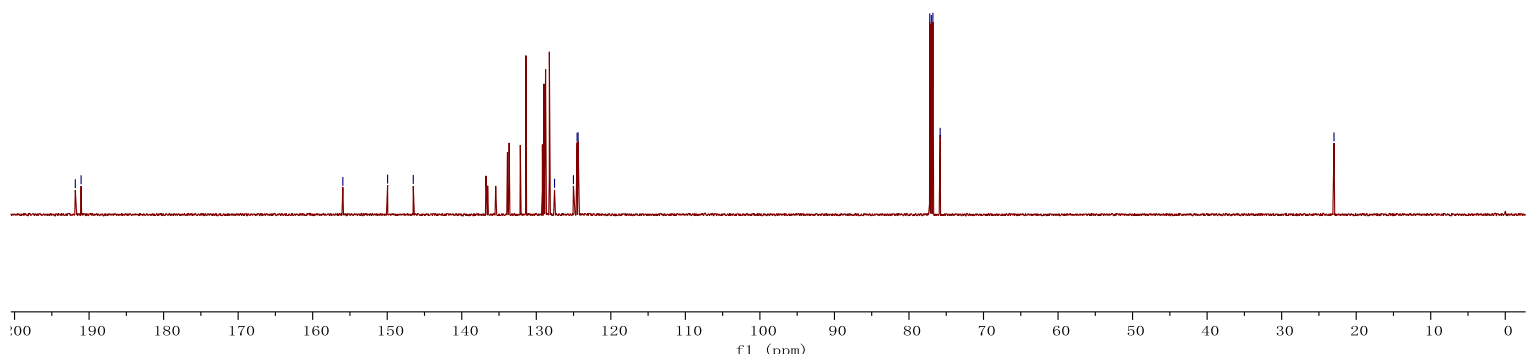

Compound $3 \mathbf{j}^{13} \mathrm{C}$ NMR $150 \mathrm{MHz}$ 


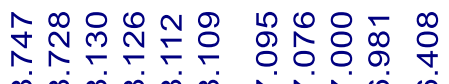

$\infty \infty \infty \infty \infty \infty \cdots$

$\rightarrow \stackrel{0}{1}$

ת)

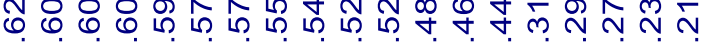

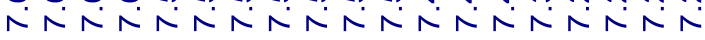
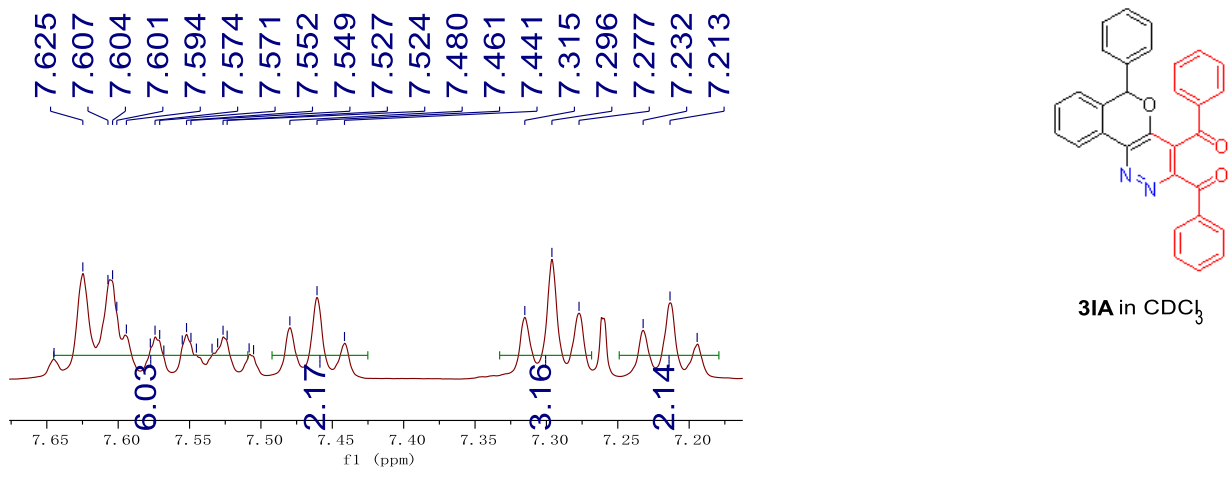

3IA in $\mathrm{CDCl}_{3}$

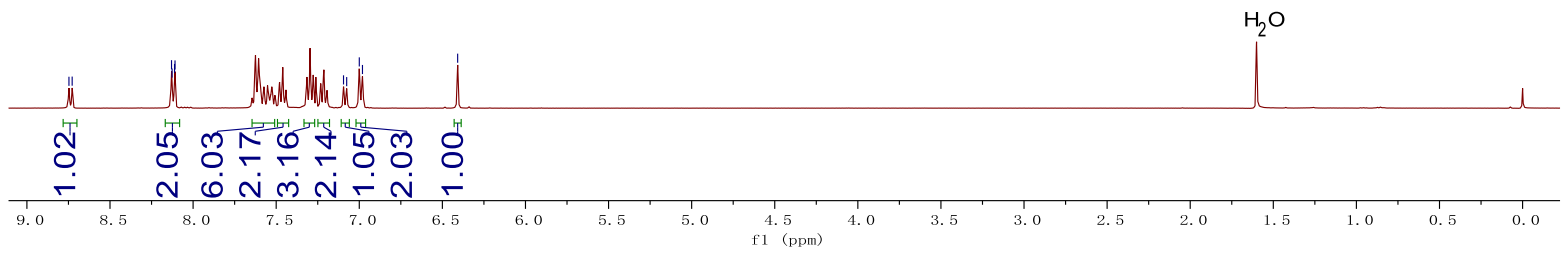

Compound 3 IA ${ }^{1} \mathrm{H}$ NMR $400 \mathrm{MHz}$

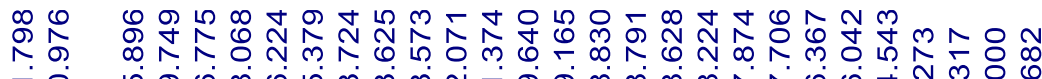

Гே
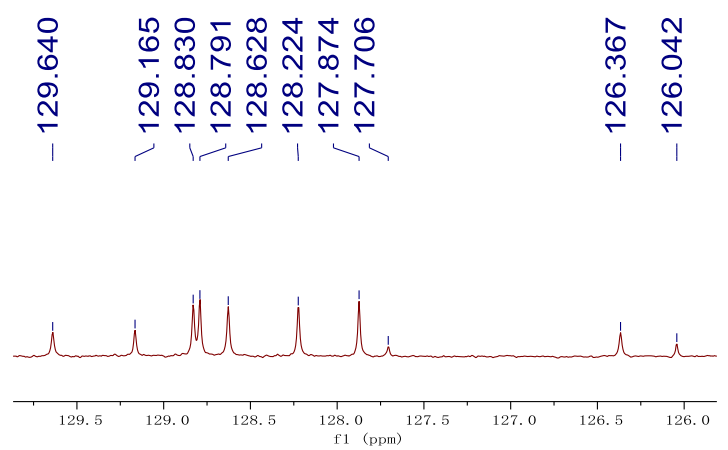

$\mathrm{CDCl}_{3}$
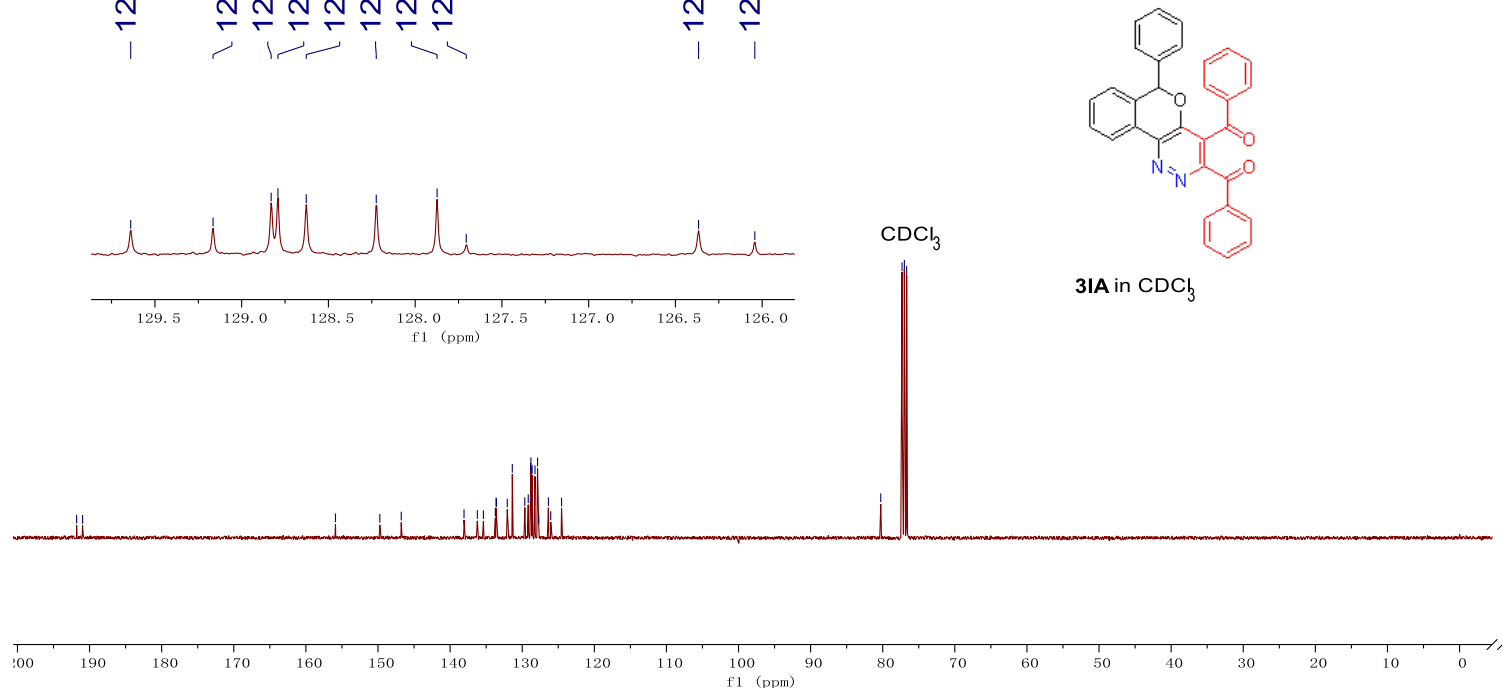

Compound 3 IA ${ }^{13} \mathrm{C}$ NMR $100 \mathrm{MHz}$ 

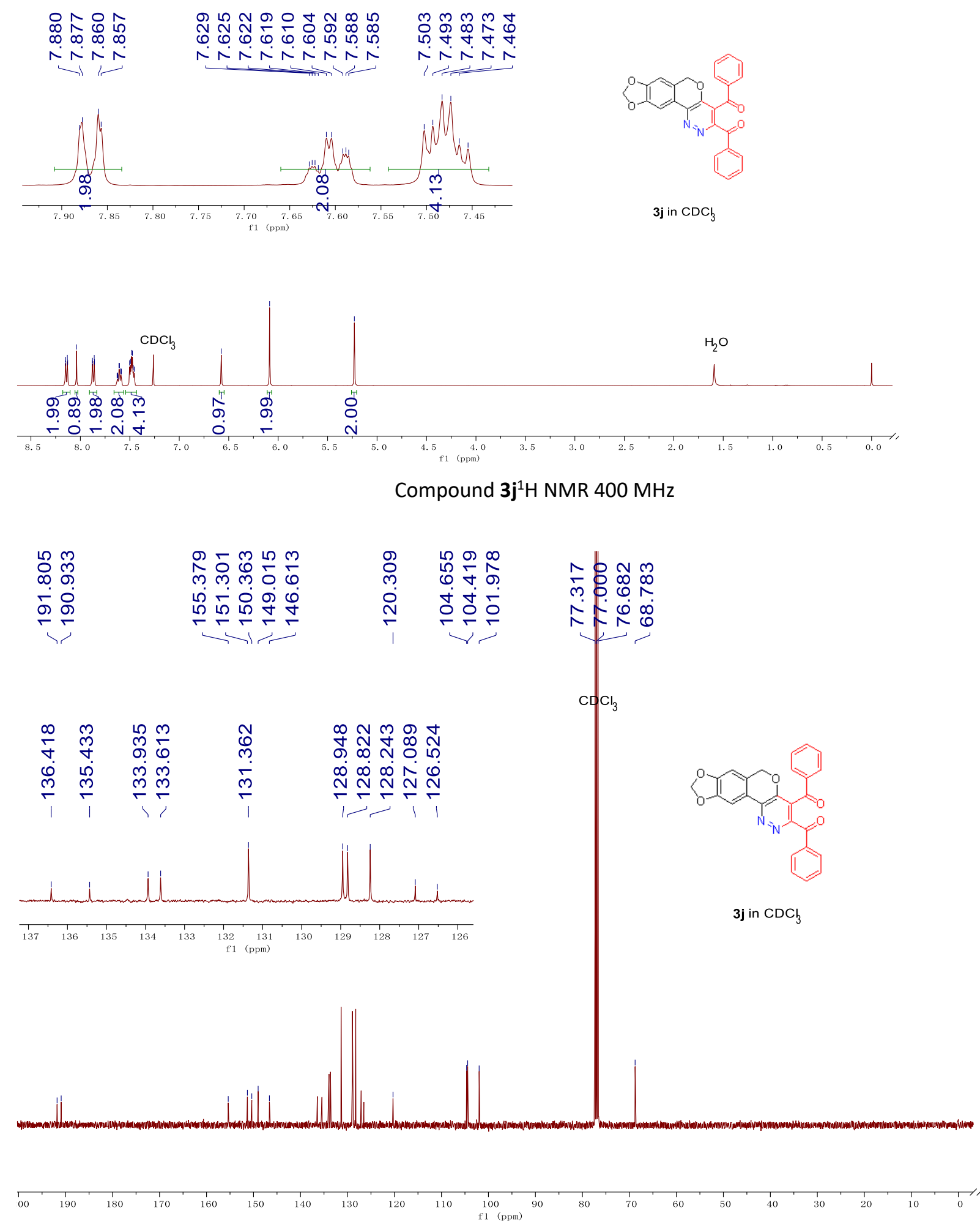

Compound 3j ${ }^{13} \mathrm{C}$ NMR $100 \mathrm{MHz}$ 


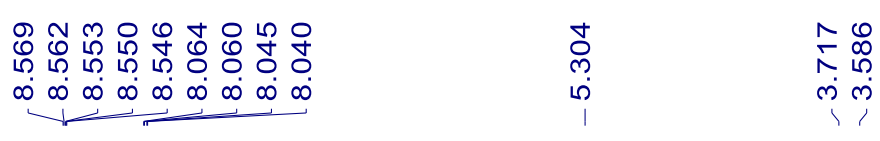

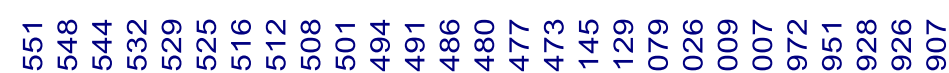

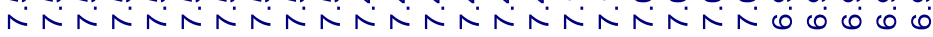

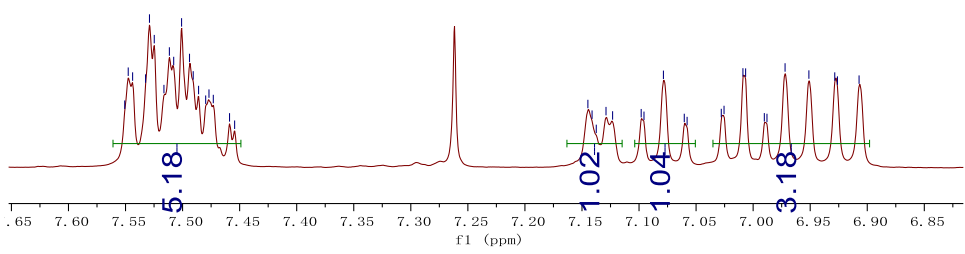

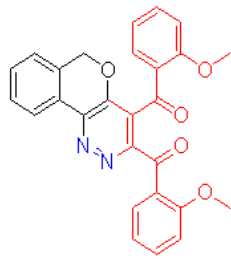

$\mathbf{3 k}$ in $\mathrm{CDCl}_{3}$

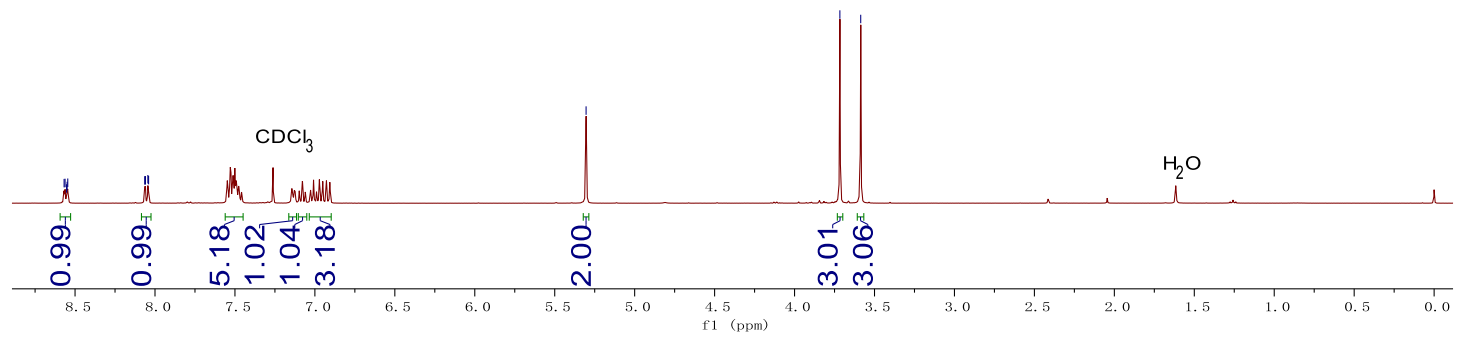

Compound $3 \mathbf{k}^{1} \mathrm{H}$ NMR $400 \mathrm{MHz}$

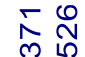

ले

ติ

i

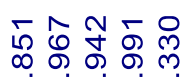

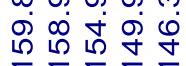

-

大

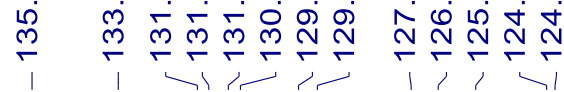

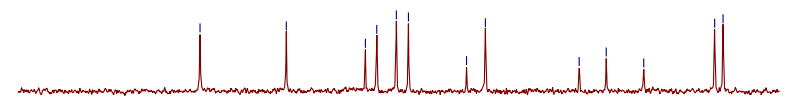

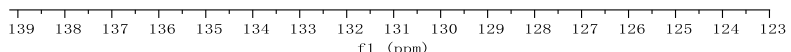

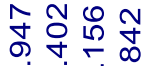

ำ ํำ

$r P^{2}$

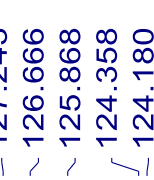

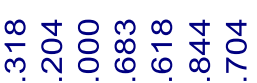

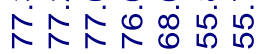
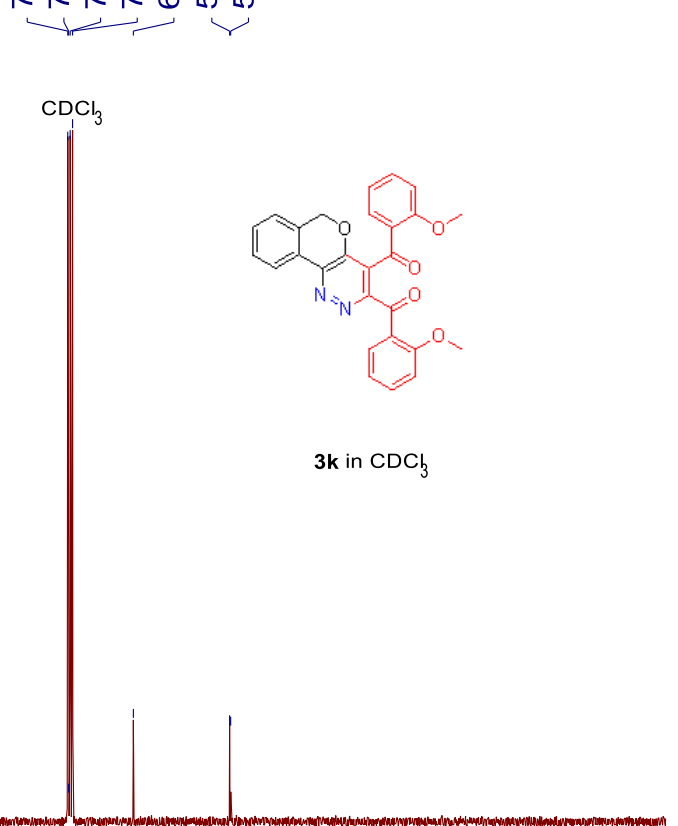

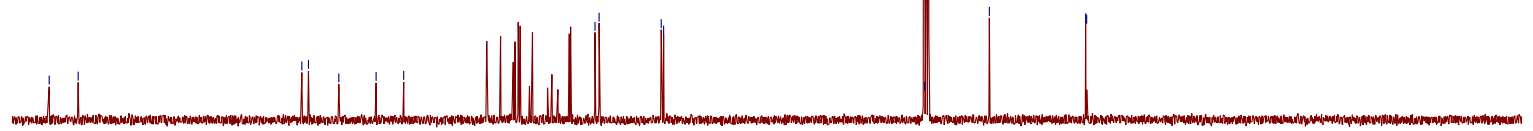

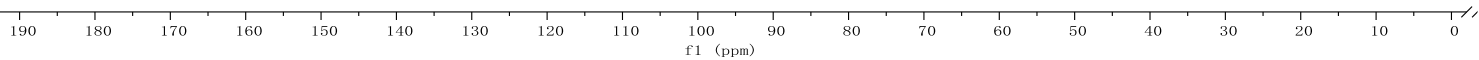

Compound $3 \mathbf{k}^{13} \mathrm{C}$ NMR $100 \mathrm{MHz}$ 


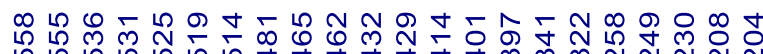
ถ

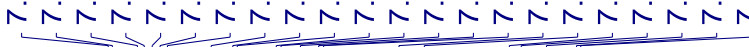

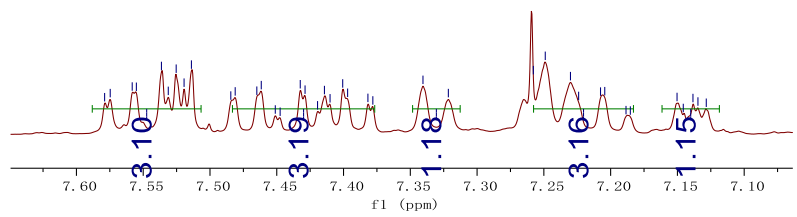

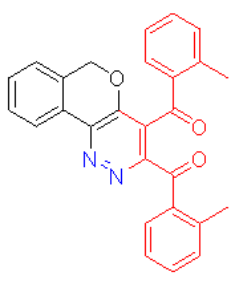

31 in $\mathrm{CDC}_{3}$

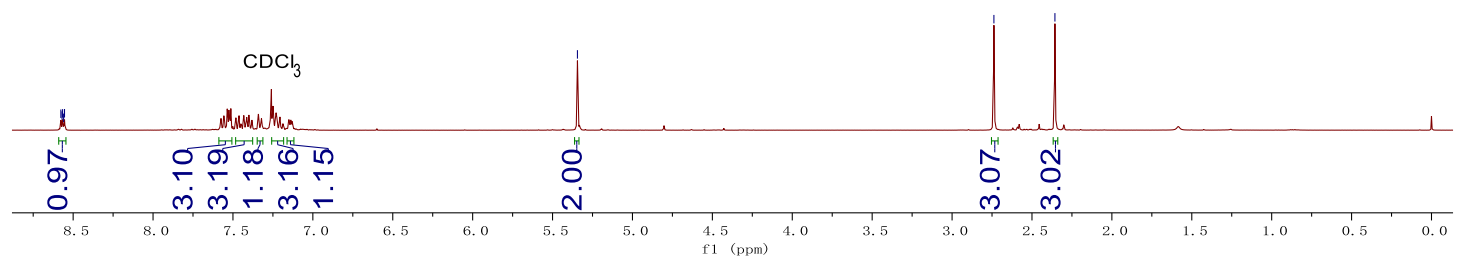

Compound $3 \mathbf{1}^{1} \mathrm{H}$ NMR $400 \mathrm{MHz}$

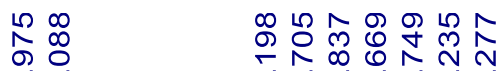

宁哭

드요요

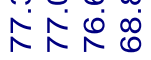

$\frac{1}{2}$

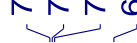

ล்
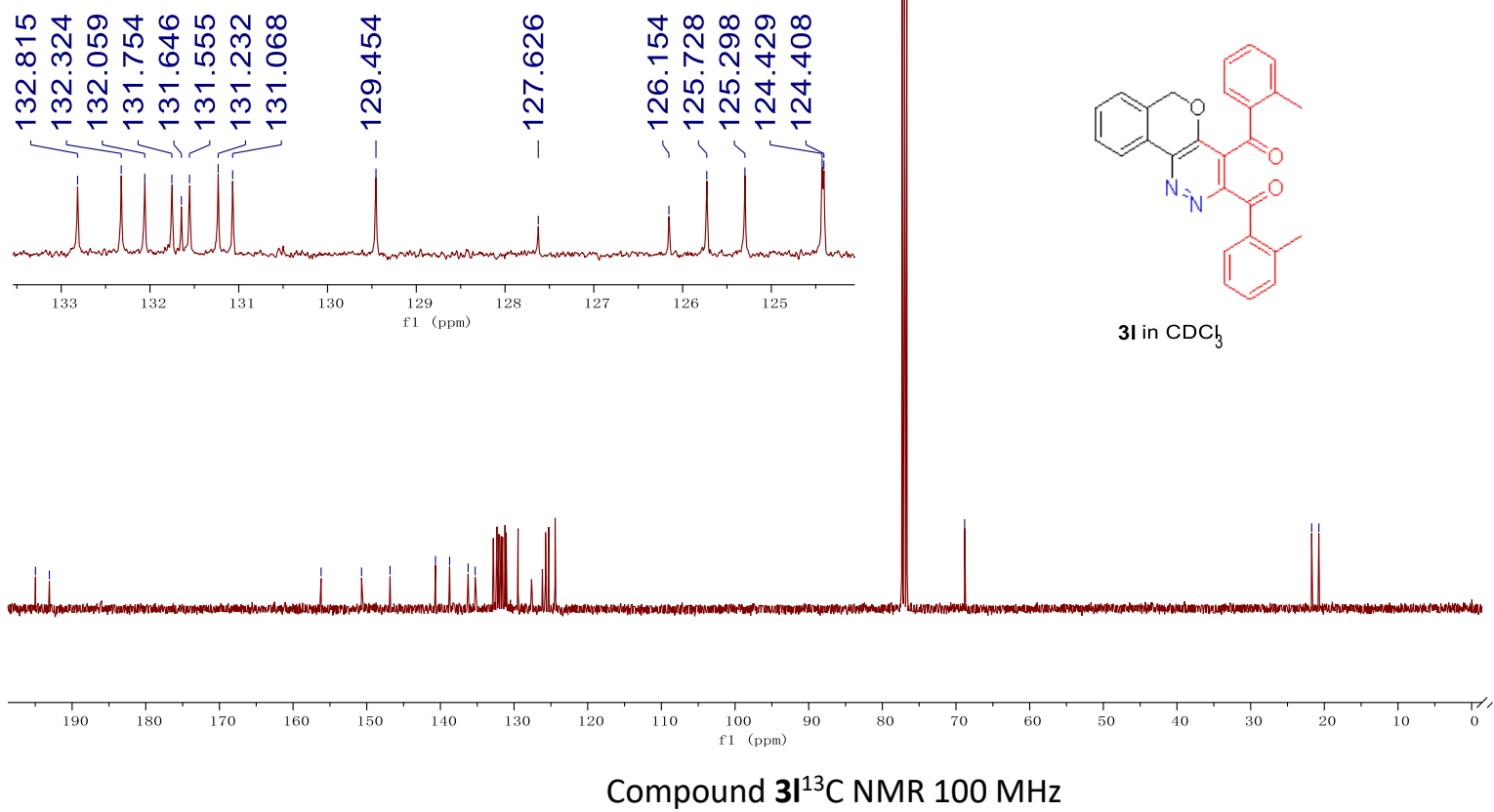
Lூ

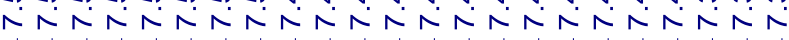

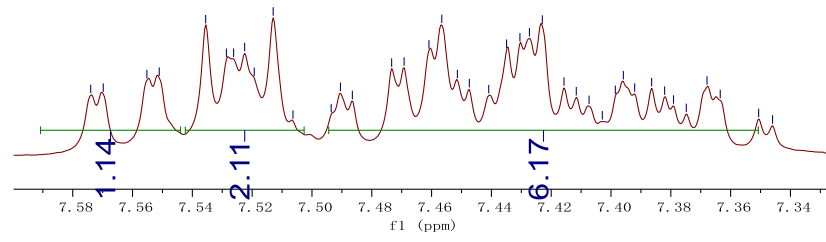

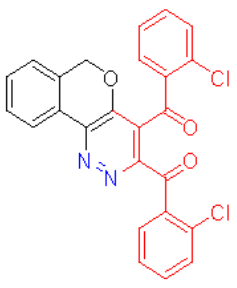

$3 \mathrm{~m}$ in $\mathrm{CDCl}_{3}$

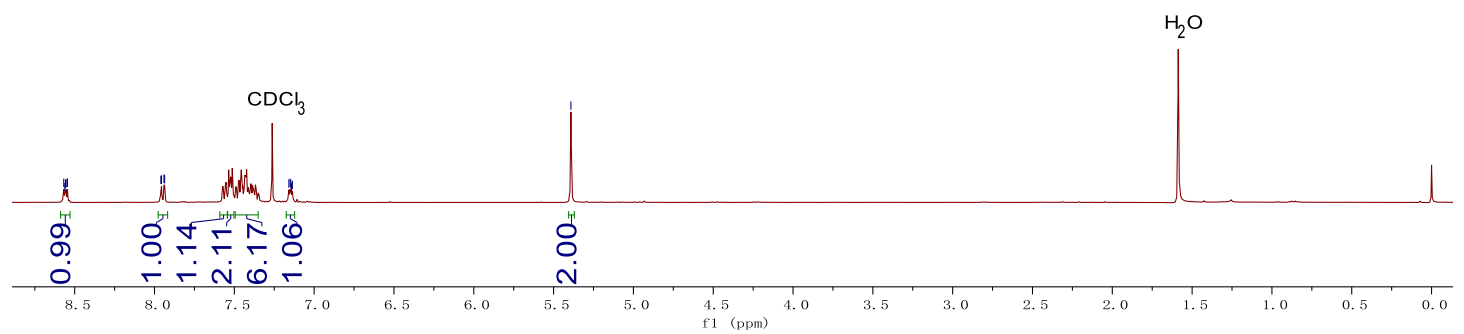

Compound $3 \mathrm{~m}^{1} \mathrm{H}$ NMR $400 \mathrm{MHz}$

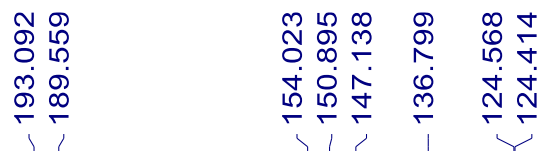

$\sum_{\substack{1 \\ \infty}}$

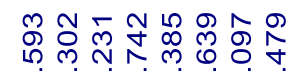

峁

\section{is}

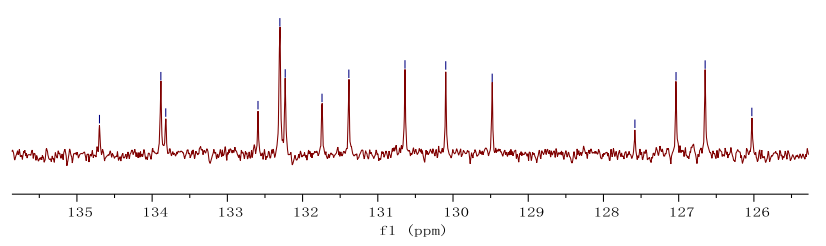

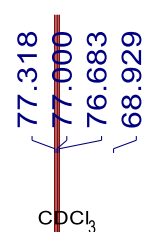

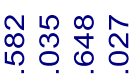

슨

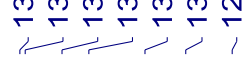

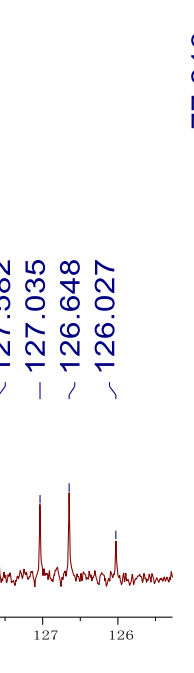

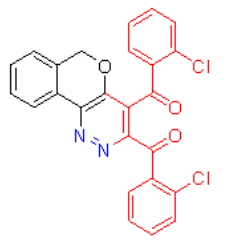

$3 \mathrm{~m}$ in $\mathrm{CDC}_{3}$
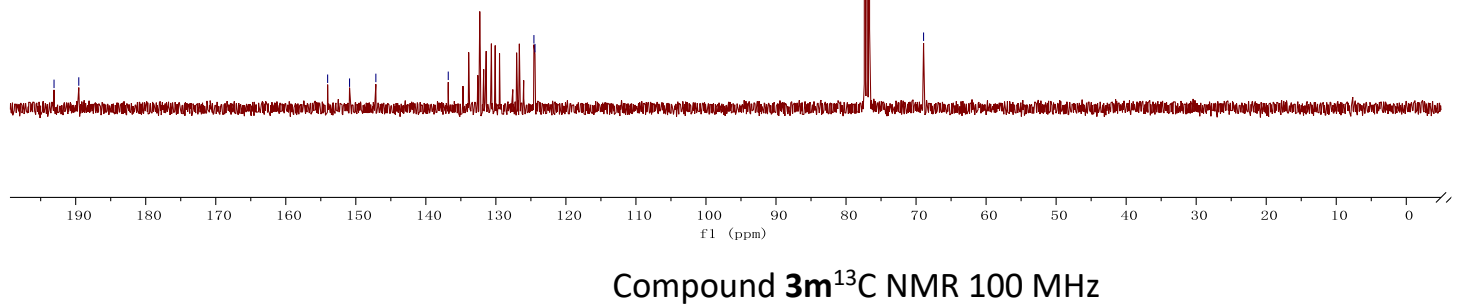
Г

NNNNNNNNNNNNNNNNN

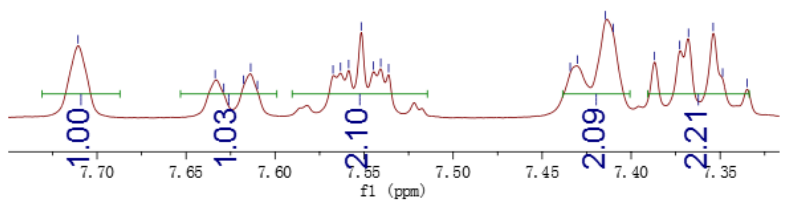

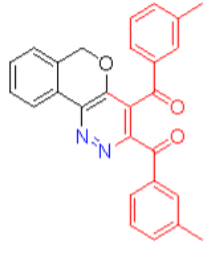

3n in $\mathrm{CDCl}_{3}$

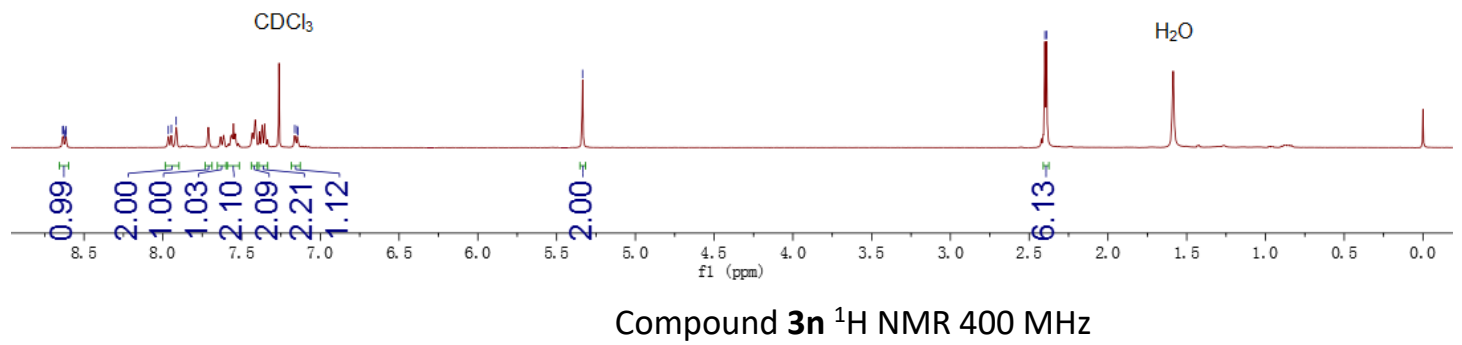
$\stackrel{\infty}{\frac{2}{\infty}}$

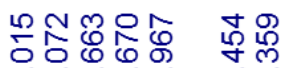

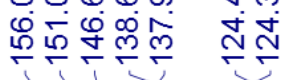
등요유
NR⿻日禸
유유
जे

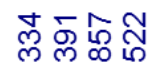

융 웅

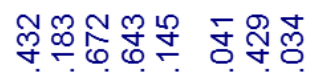

씼 쑴

लंख्ले

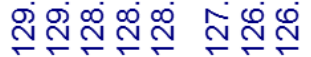
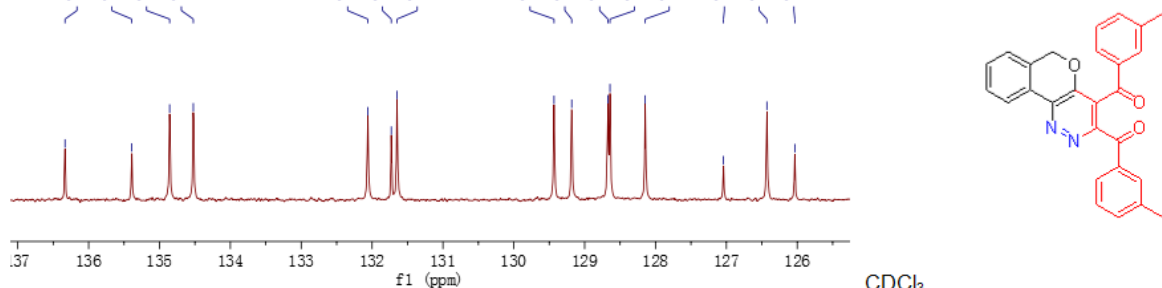

$\mathrm{CDCl}_{3}$ 3n in $\mathrm{CDCl}_{3}$

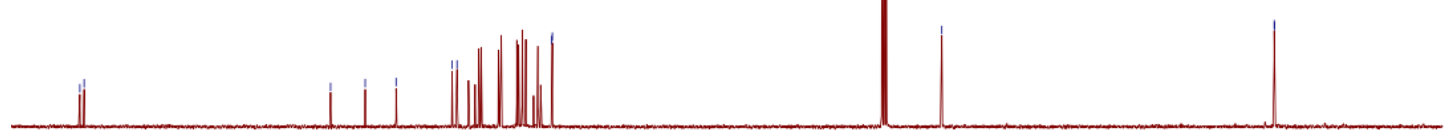

200
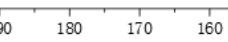

110 


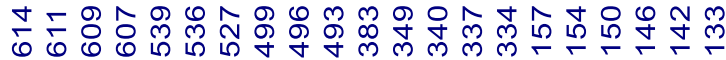

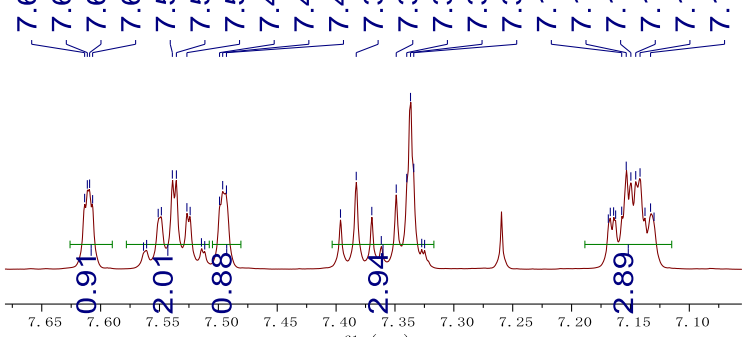

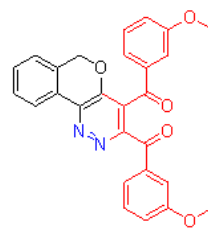

3o in $\mathrm{CDCl}_{3}$

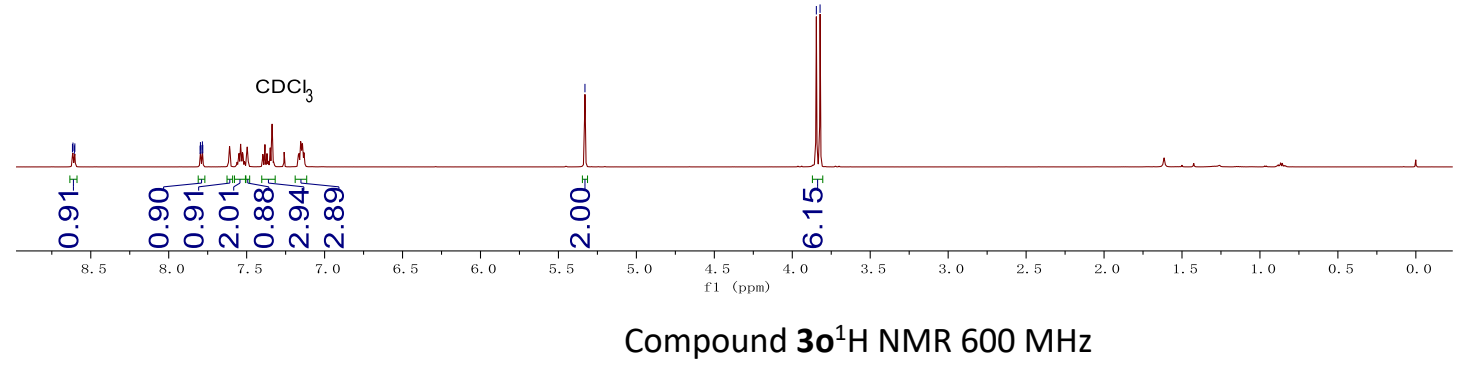

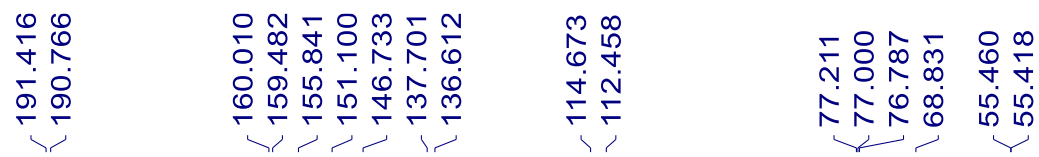

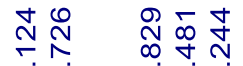

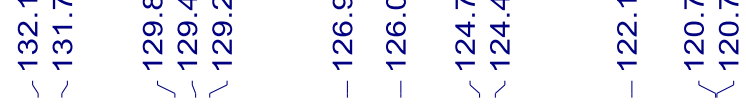
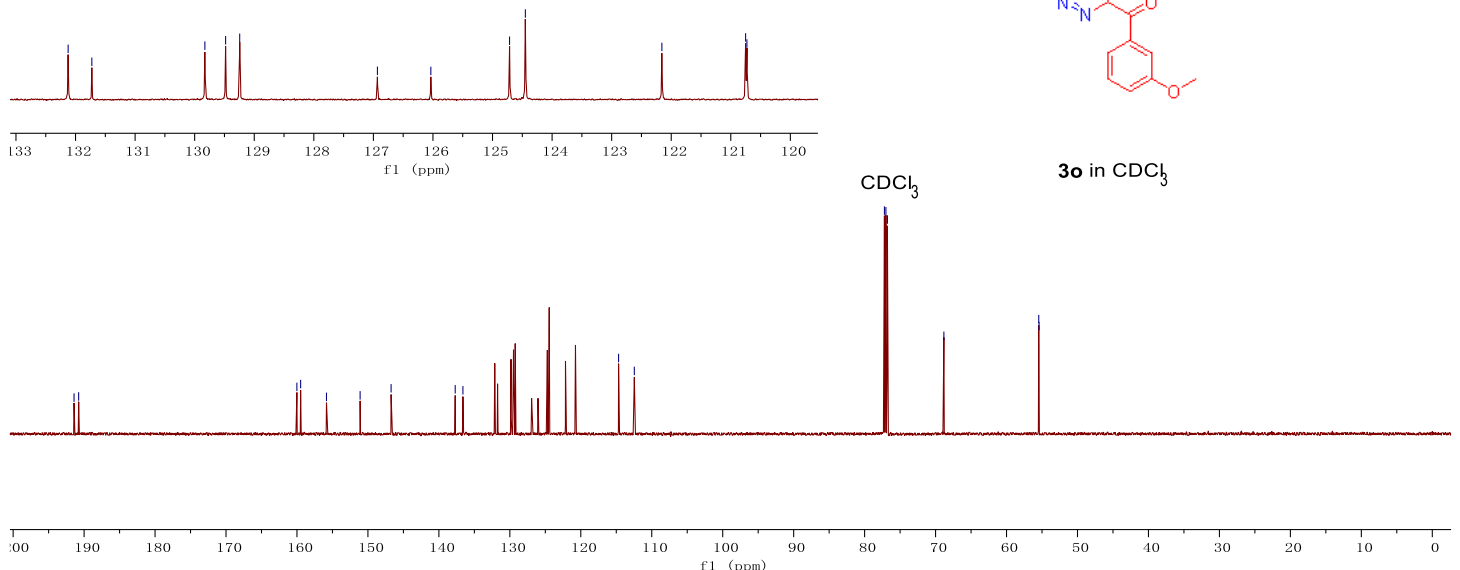

Compound $30^{13} \mathrm{C}$ NMR $150 \mathrm{MHz}$ 


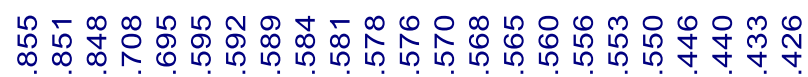
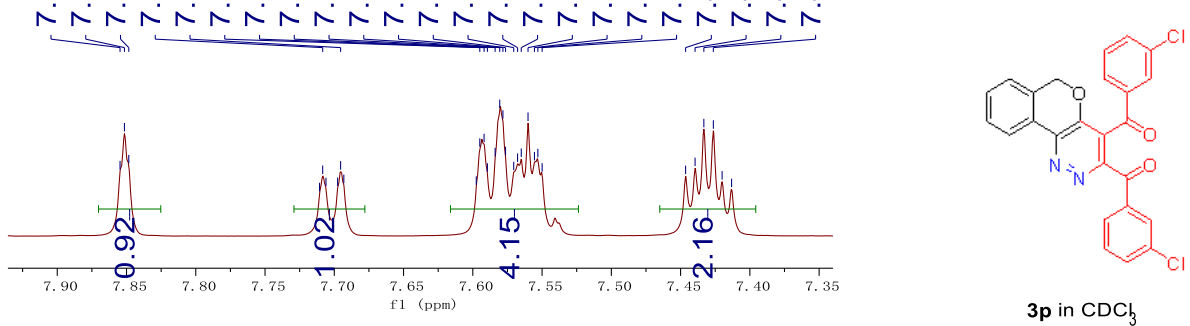

$3 \mathbf{p}$ in $\mathrm{CDC}_{3}$

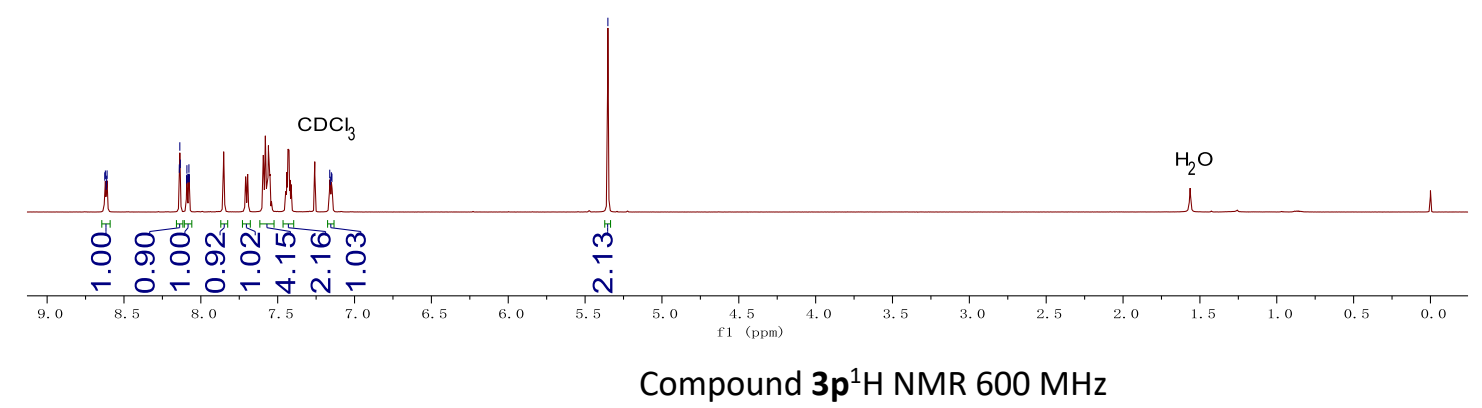

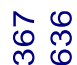

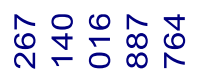

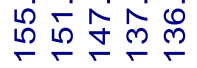
능요용
욤
i

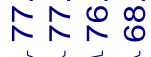
作

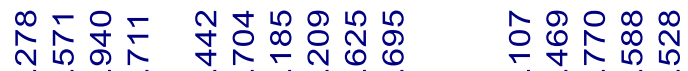

官
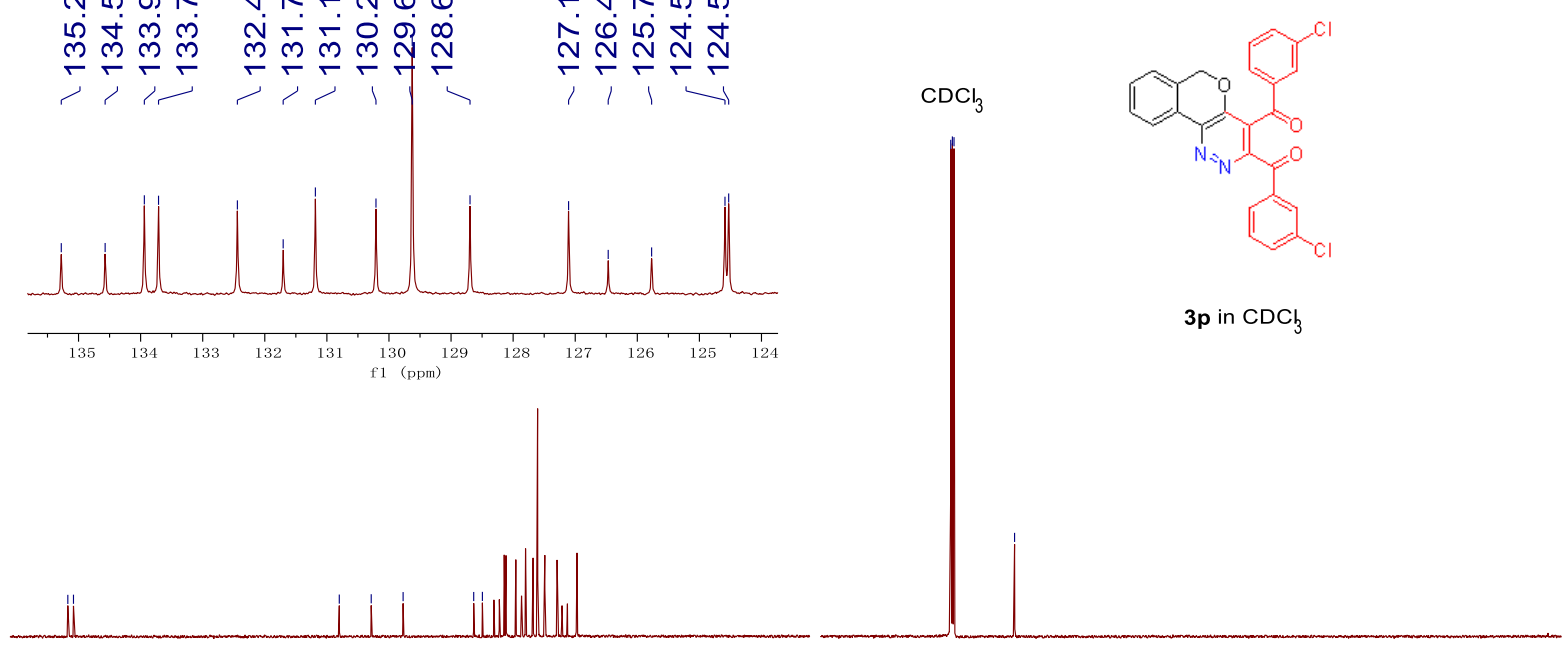

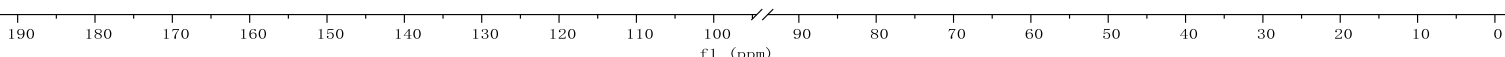

Compound $3 p^{13} \mathrm{C}$ NMR $150 \mathrm{MHz}$ 

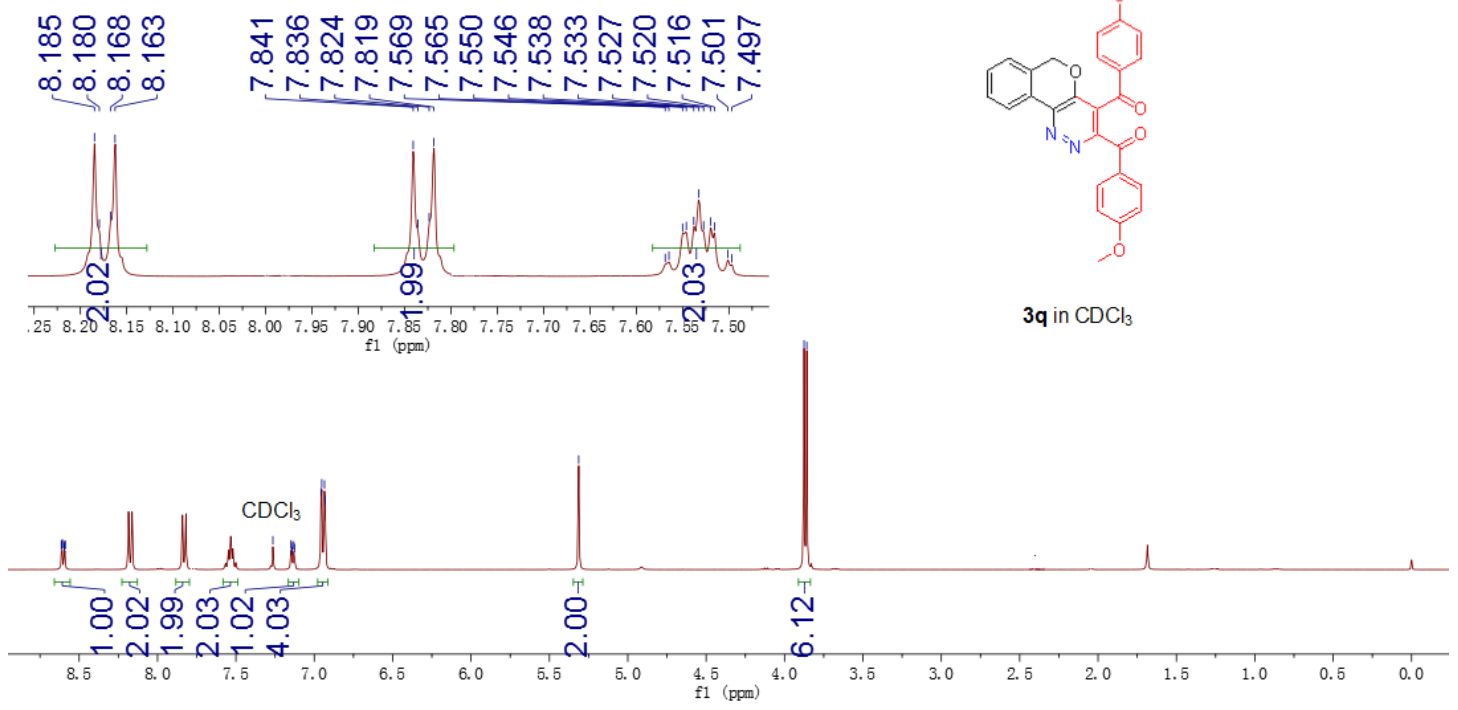

Compound $3 \mathbf{q}^{1} \mathrm{H}$ NMR $400 \mathrm{MHz}$

\begin{tabular}{|c|c|c|c|c|}
\hline 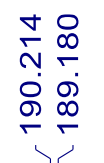 & 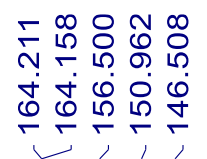 & 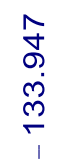 & 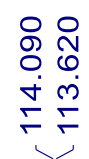 & 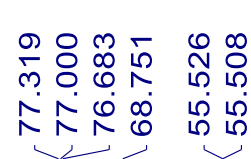 \\
\hline
\end{tabular}
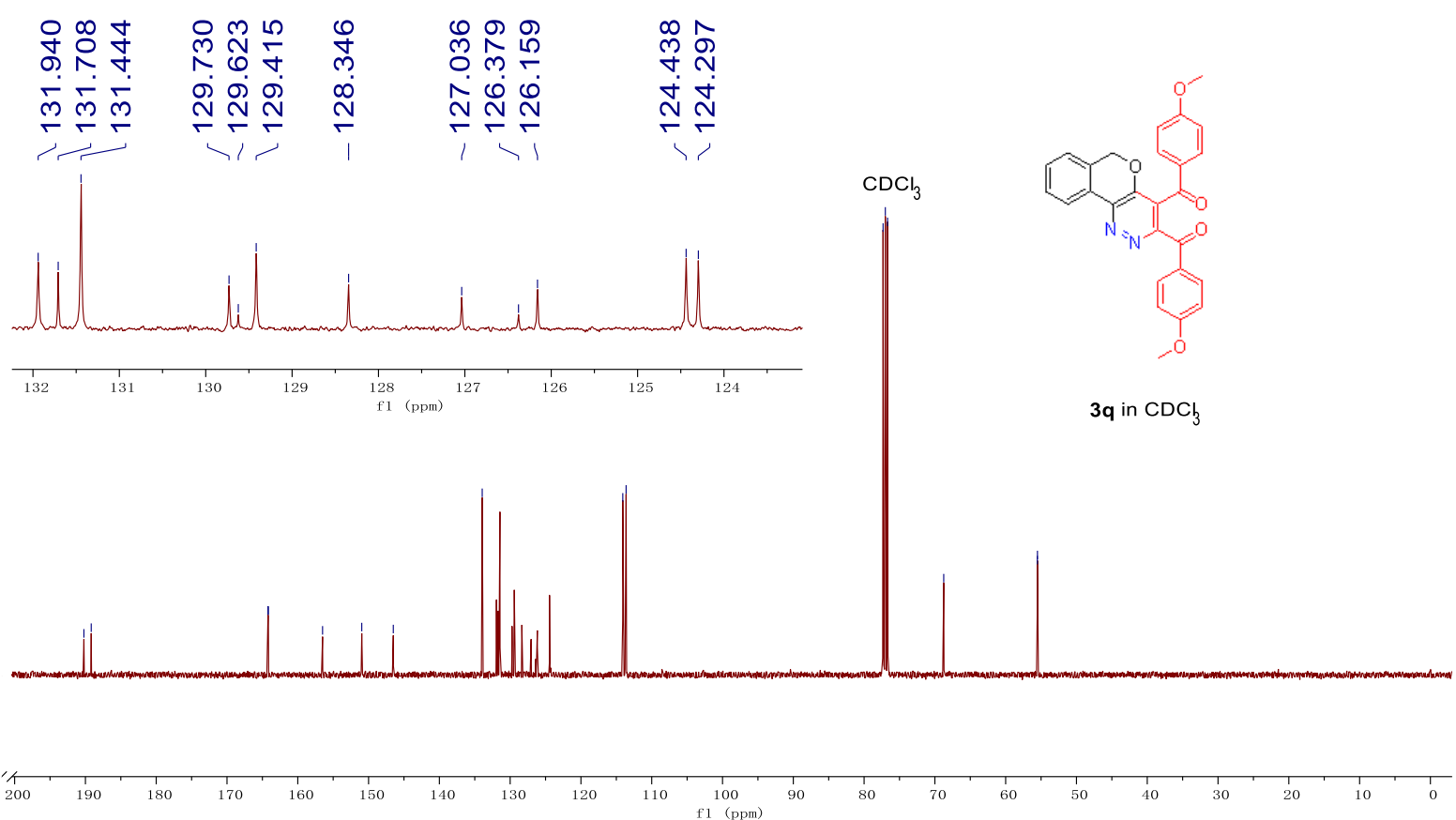

Compound $3 \mathbf{q}^{13} \mathrm{C}$ NMR $100 \mathrm{MHz}$ 


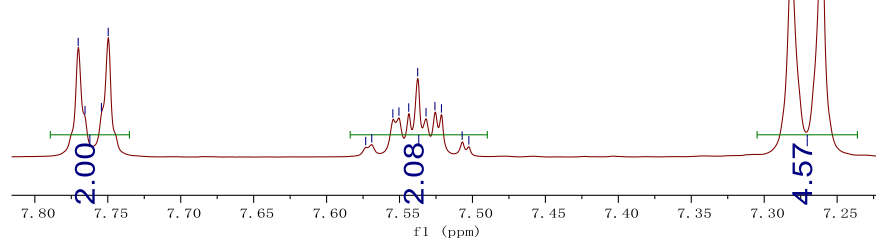

f1 (ppm)

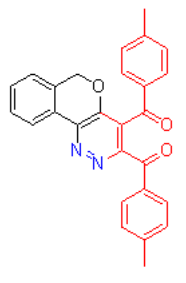

$3 \mathbf{r}$ in $\mathrm{CDC}_{3}$

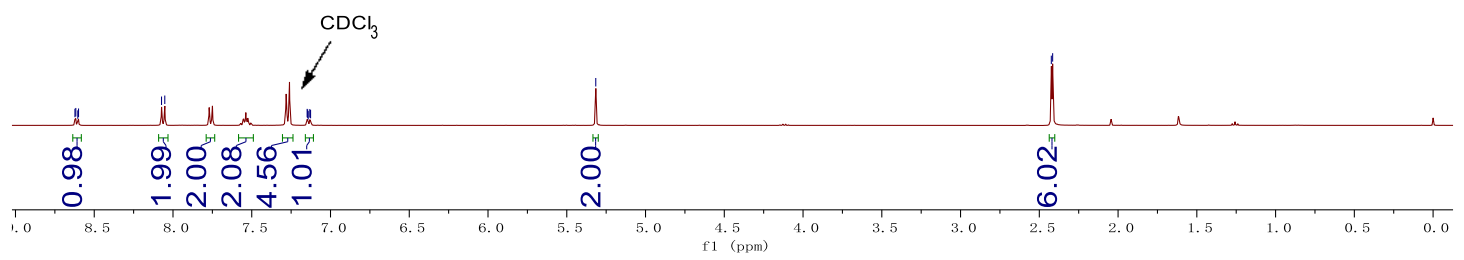

Compound $3 r^{1} \mathrm{H}$ NMR $400 \mathrm{MHz}$

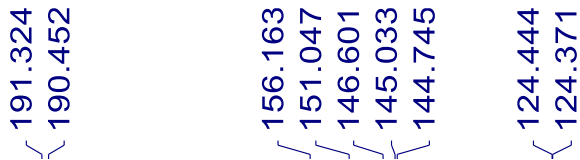

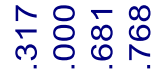

ヘト゚்

$\underset{\infty}{N} \frac{0}{\infty}$

ড̀
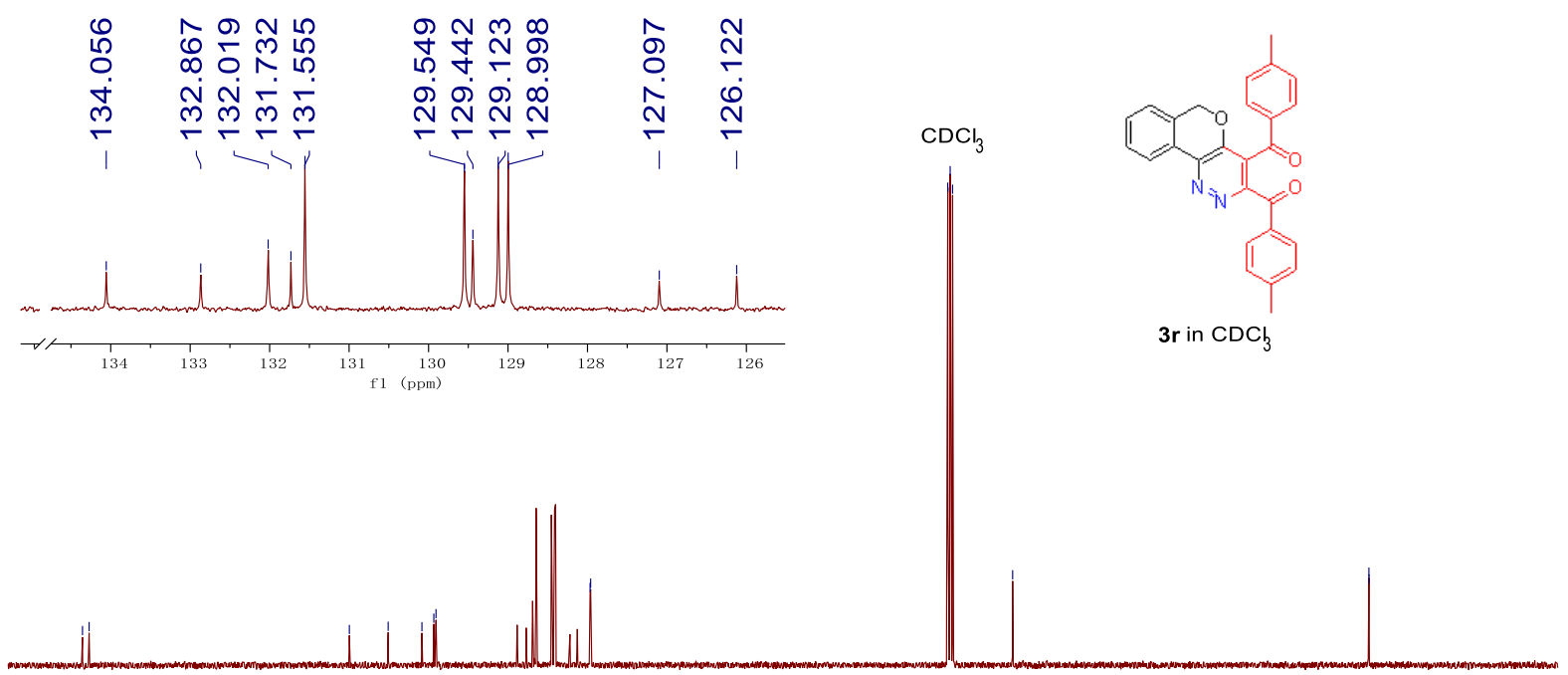

Compound $3 r^{13} \mathrm{C}$ NMR $100 \mathrm{MHz}$ 

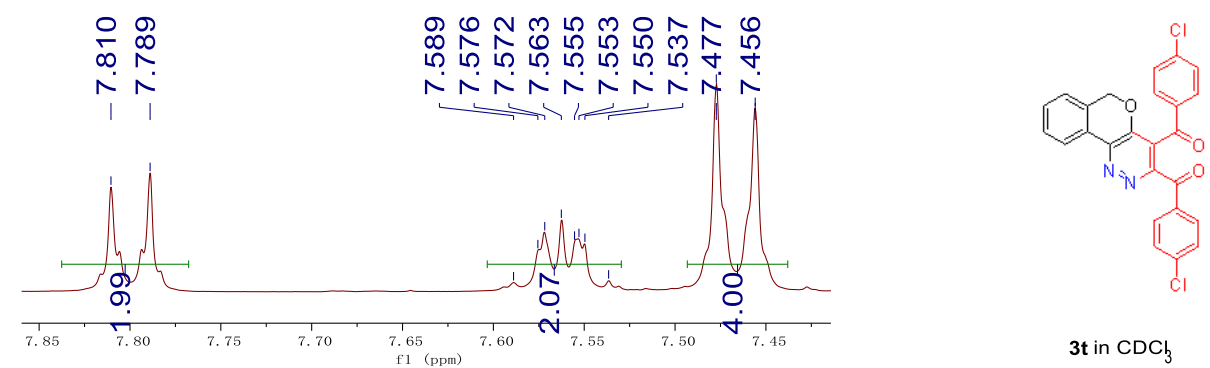

3t in $\mathrm{CDCl}_{3}$

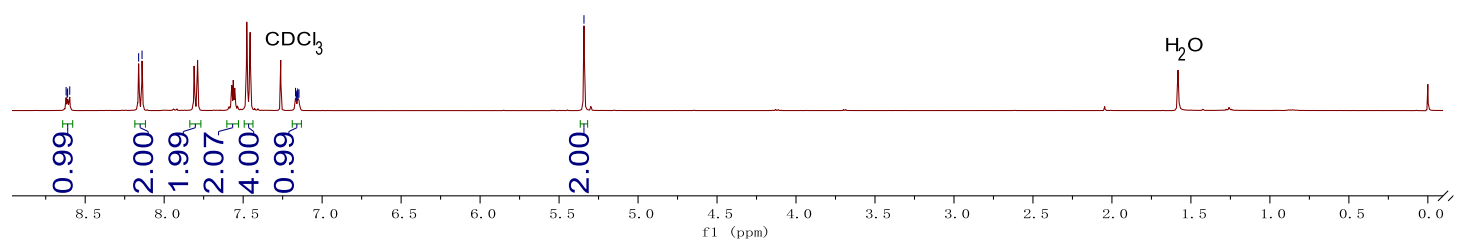

Compound $3 \mathrm{t}^{1} \mathrm{H}$ NMR $400 \mathrm{MHz}$

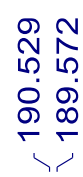

L

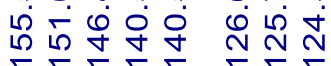

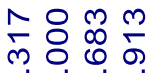

กิ่

人N

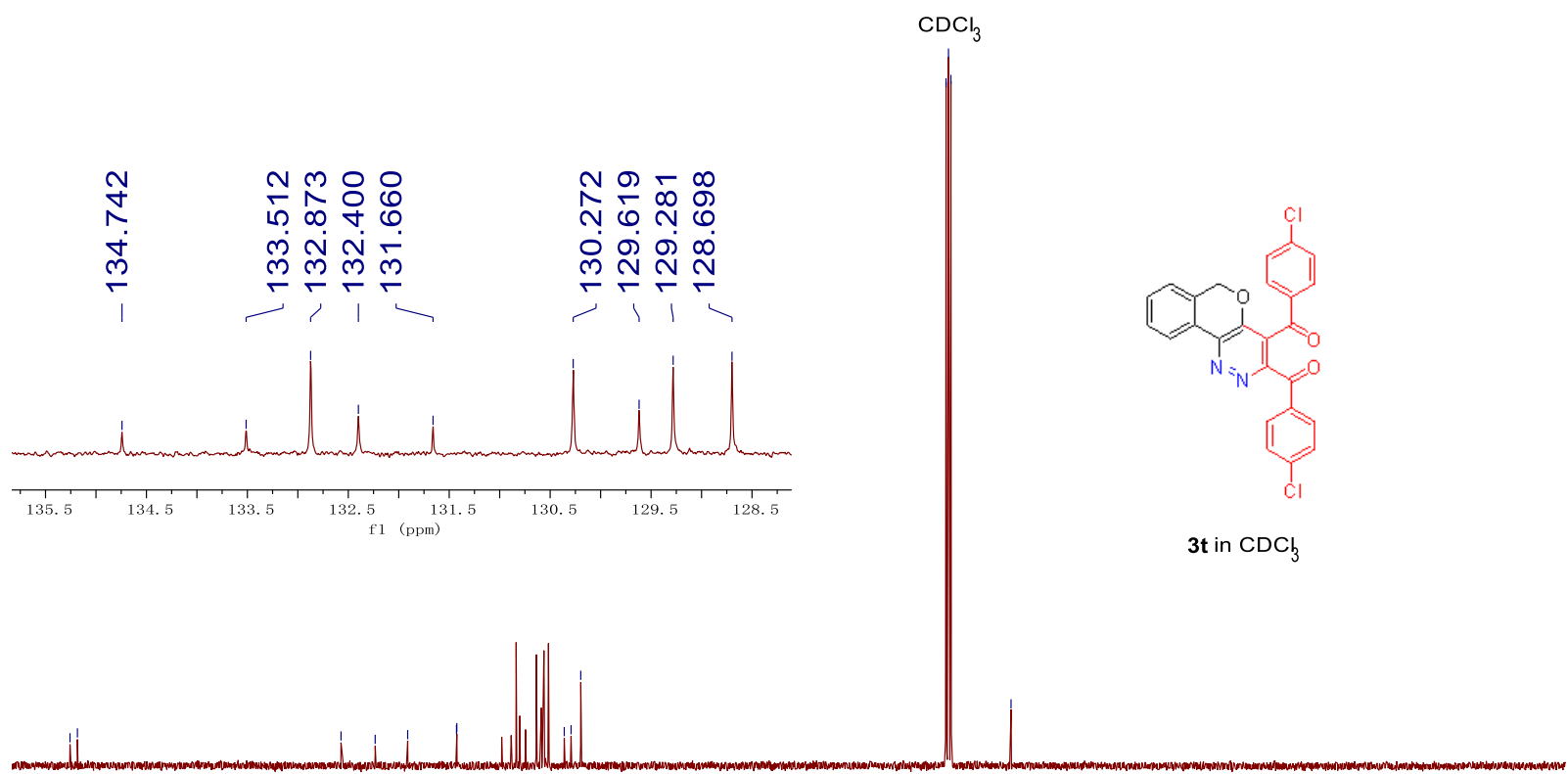

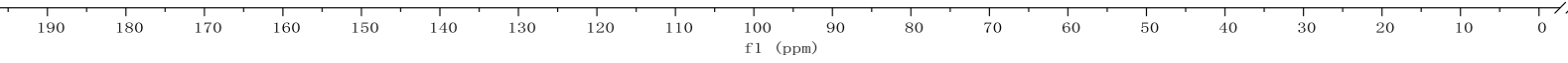

Compound $3 \mathrm{t}^{13} \mathrm{C}$ NMR $100 \mathrm{MHz}$ 


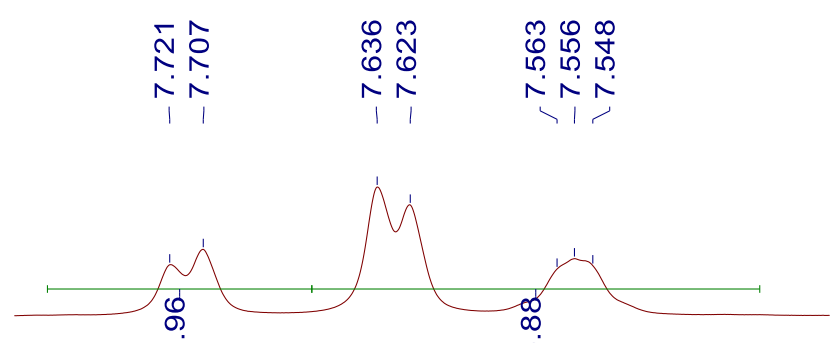

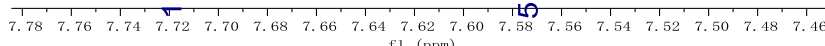

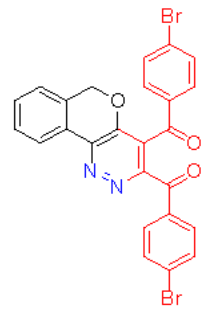

$3 \mathbf{u}$ in $\mathrm{CDC}_{3}$

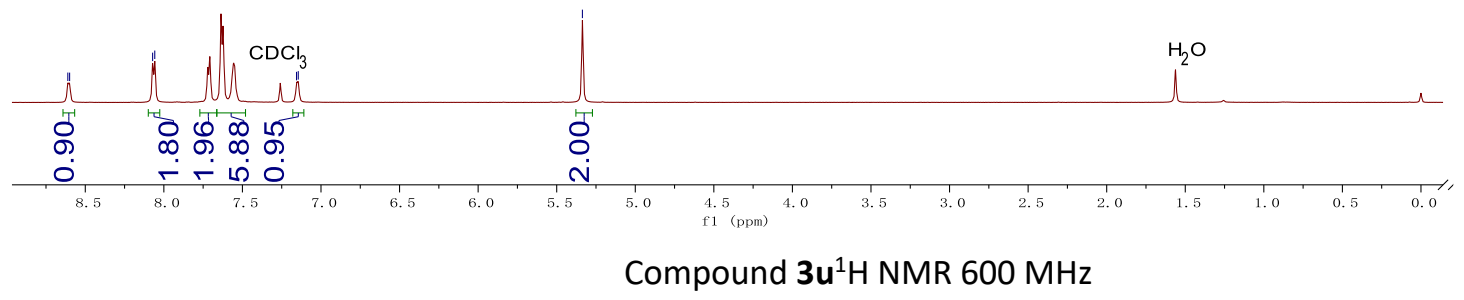

กิ

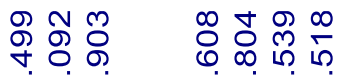

官

$\stackrel{N}{\mathrm{~N}} 8 \stackrel{\infty}{\circ} \stackrel{\infty}{\sim} \stackrel{\infty}{\sim}$

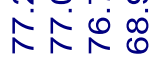

ஓं

1)

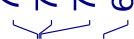

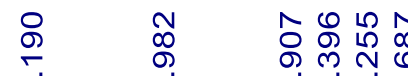

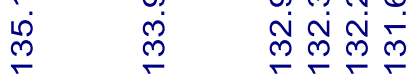

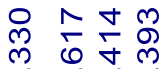

ํํㄴ 욘

$\mathrm{CDCl}_{3}$

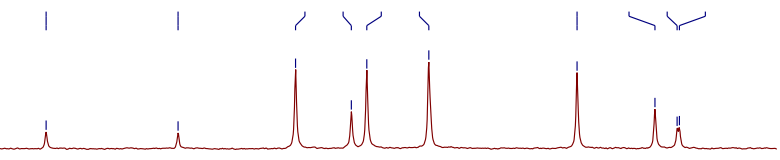

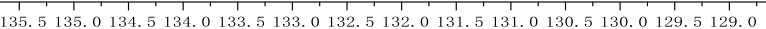

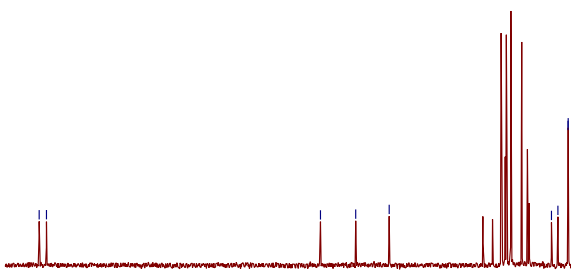

$3 \mathbf{u}$ in $\mathrm{CDCl}_{3}$

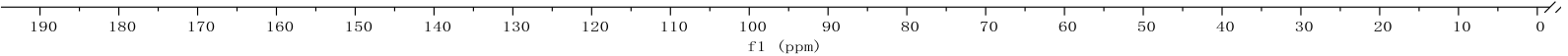

Compound $3 \mathbf{u}^{13} \mathrm{C}$ NMR $150 \mathrm{MHz}$ 

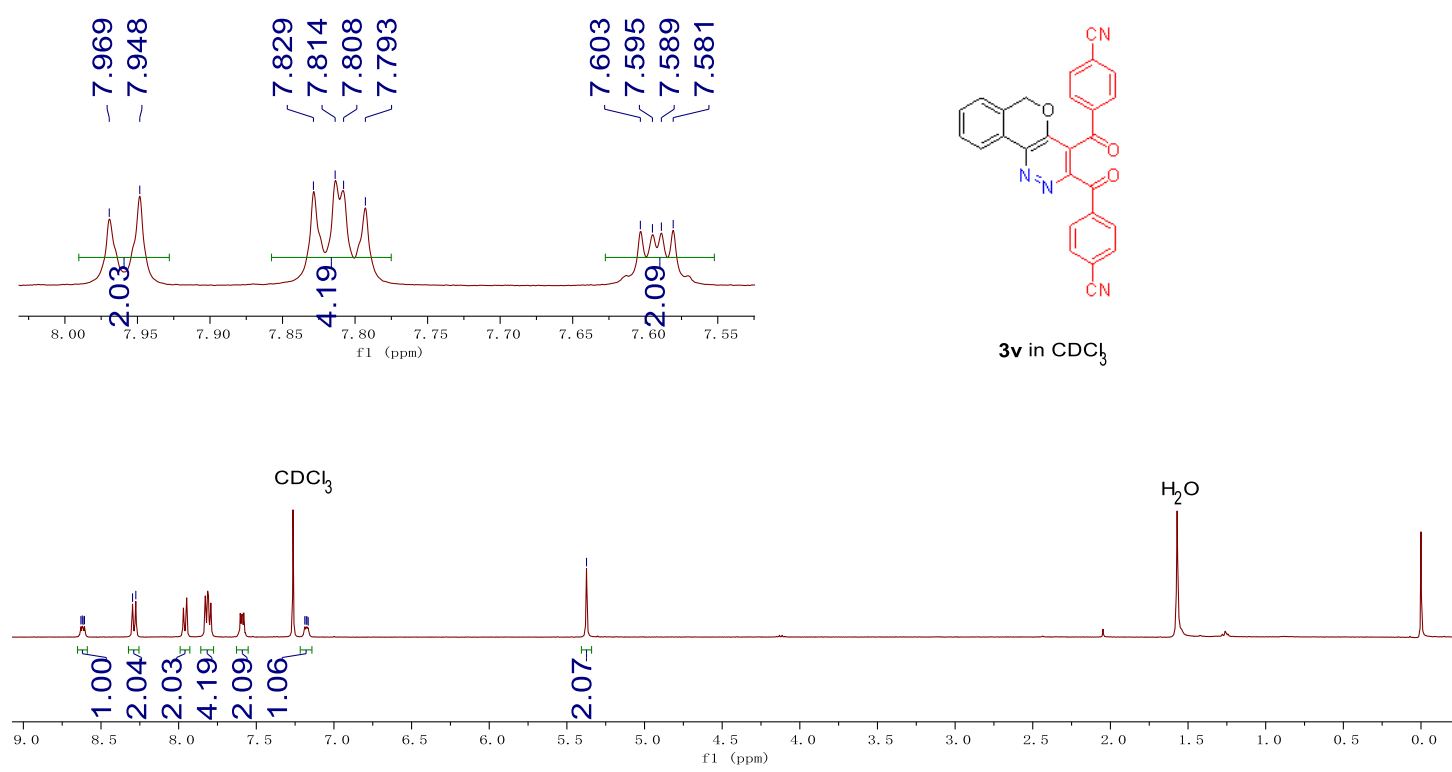

Compound $3 v^{1} \mathrm{H}$ NMR $400 \mathrm{MHz}$

\begin{tabular}{|c|c|c|}
\hline$\stackrel{\sim}{\sim} \stackrel{20}{5}$ & 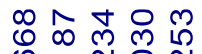 & 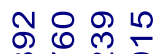 \\
\hline $\begin{array}{ll}m & 0 \\
0 & 0\end{array}$ & 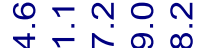 & 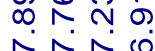 \\
\hline & & \\
\hline
\end{tabular}

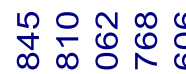

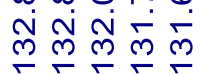

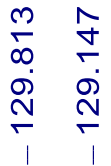

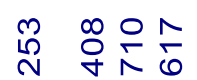

$\stackrel{\leftrightarrow}{\sim} \stackrel{i}{\sim} \stackrel{\dot{\sim}}{\sim}$
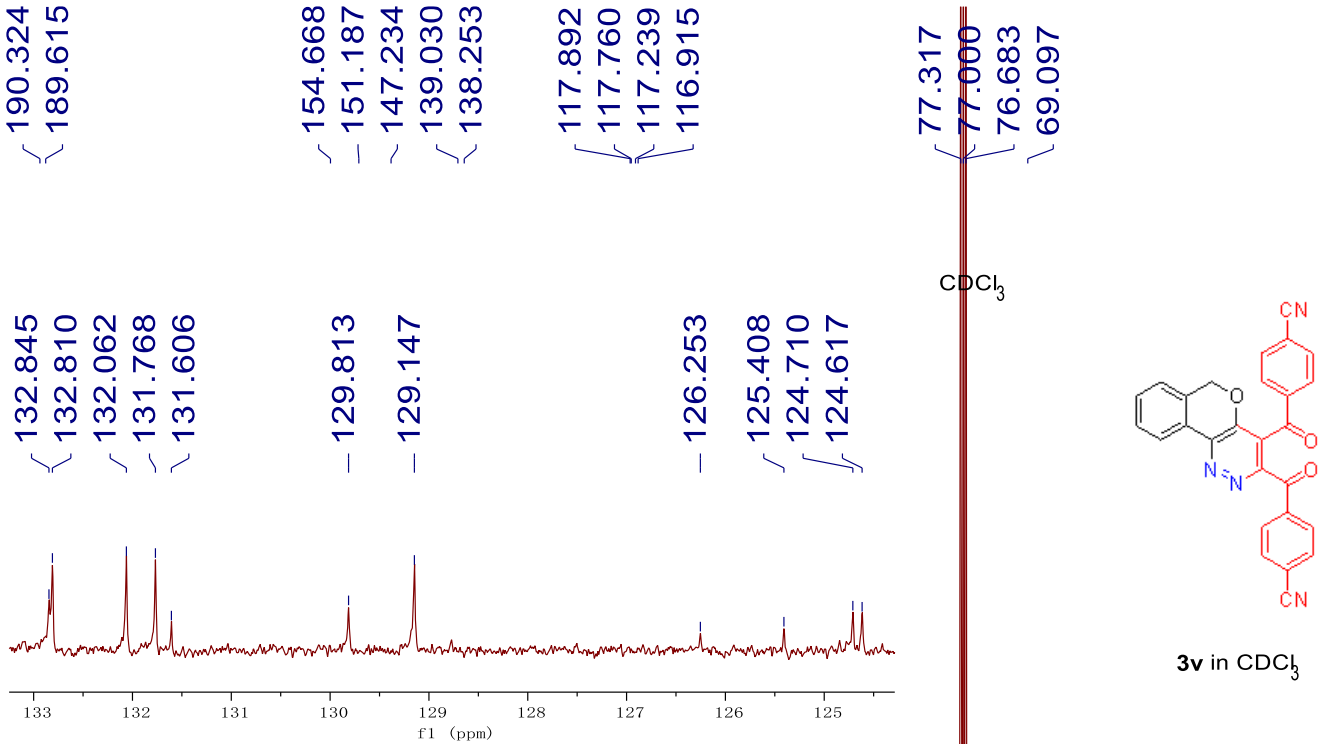

$3 v$ in $\mathrm{CDCl}_{3}$
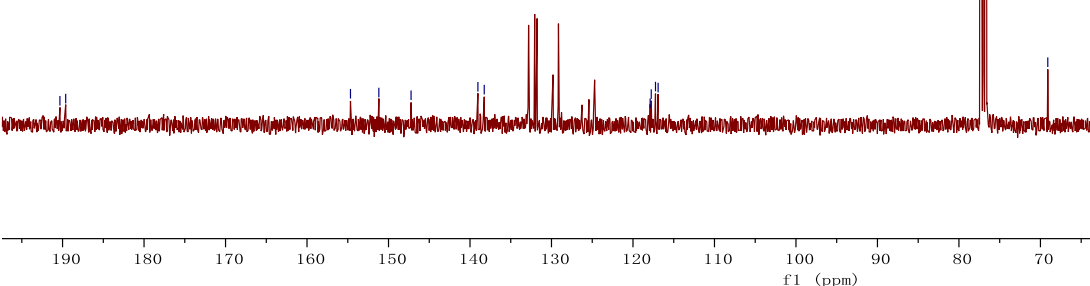


\begin{tabular}{|c|c|}
\hline 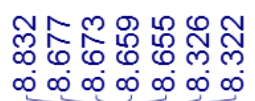 & 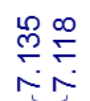 \\
\hline
\end{tabular}

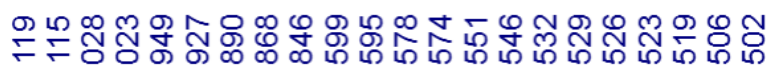
$\infty \infty \infty \infty N N N$ N
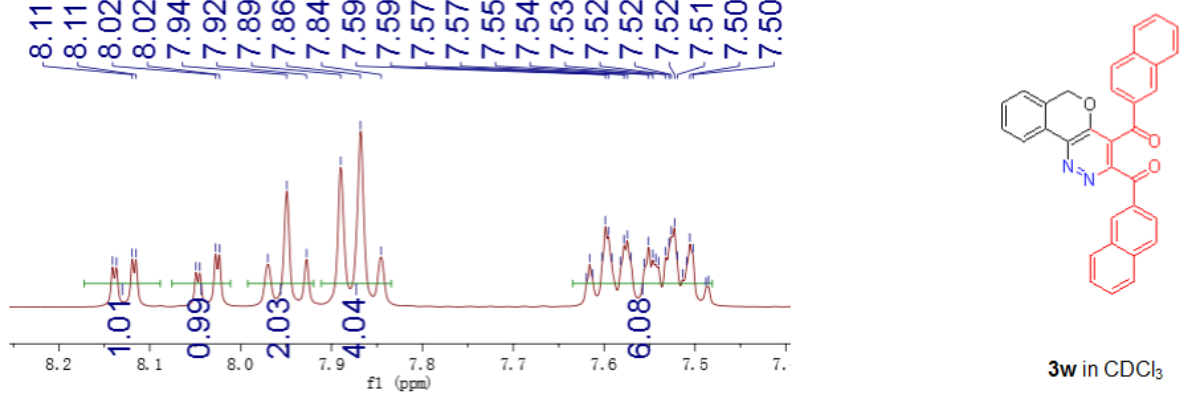

3 in $\mathrm{CDCl}_{3}$

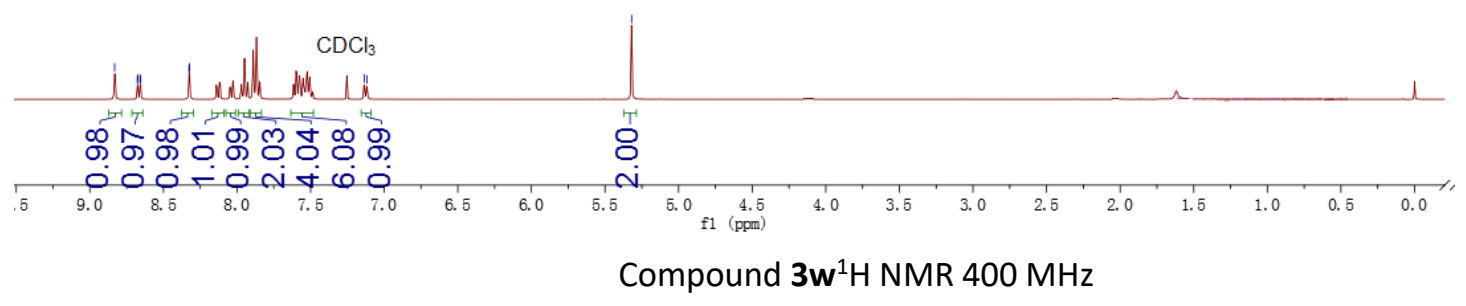

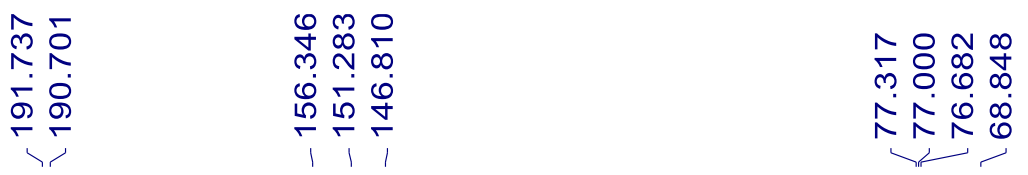

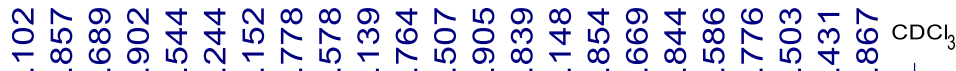
లైల్ల

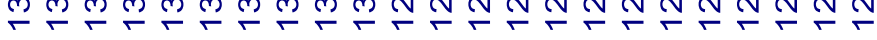
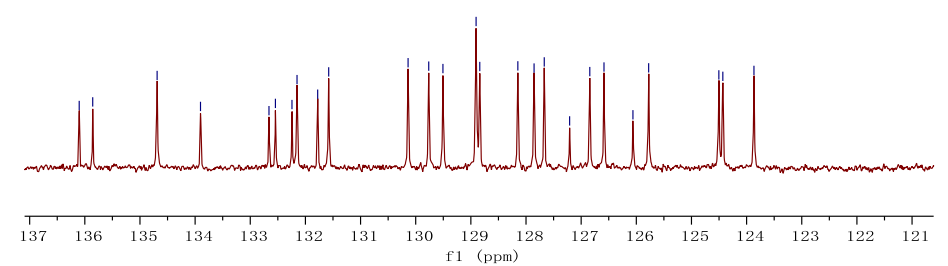

$3 \mathbf{w}$ in $\mathrm{CDCl}$

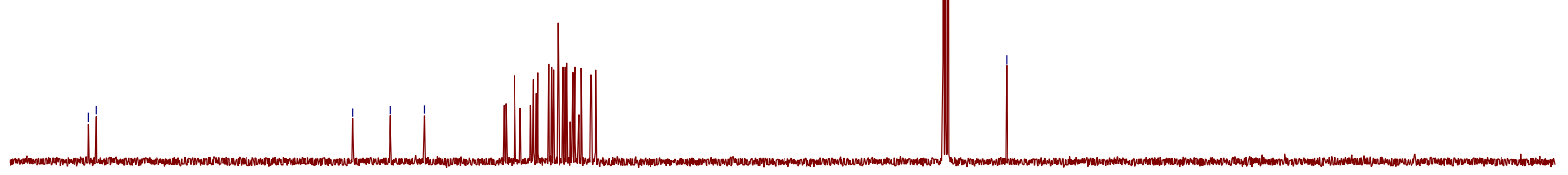

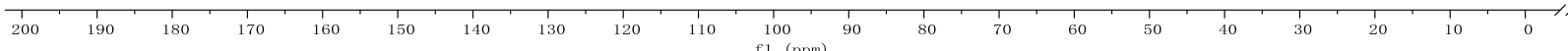

Compound $3 \mathbf{w}^{13} \mathrm{C}$ NMR $100 \mathrm{MHz}$ 


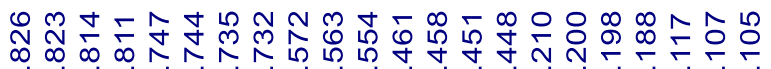

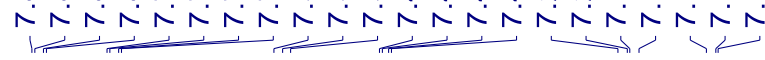
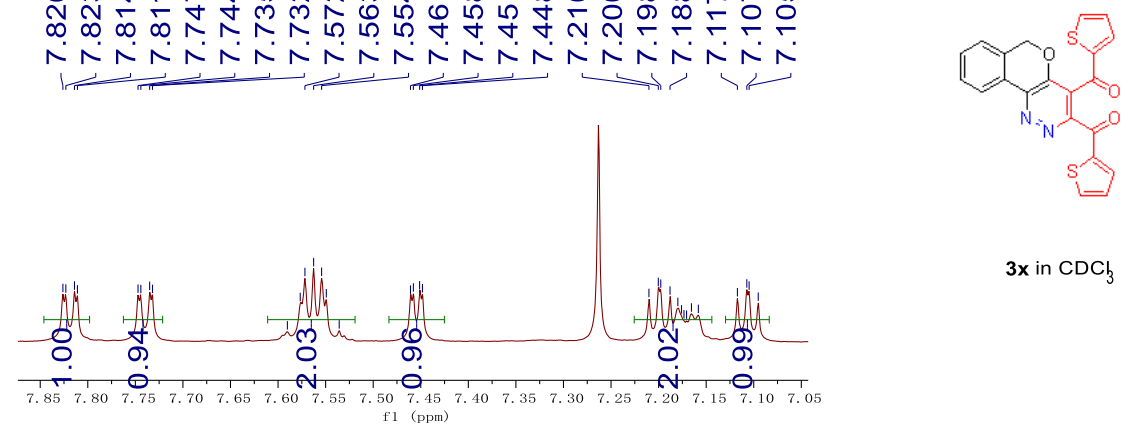

$3 \mathbf{x}$ in $\mathrm{CDC}_{3}$

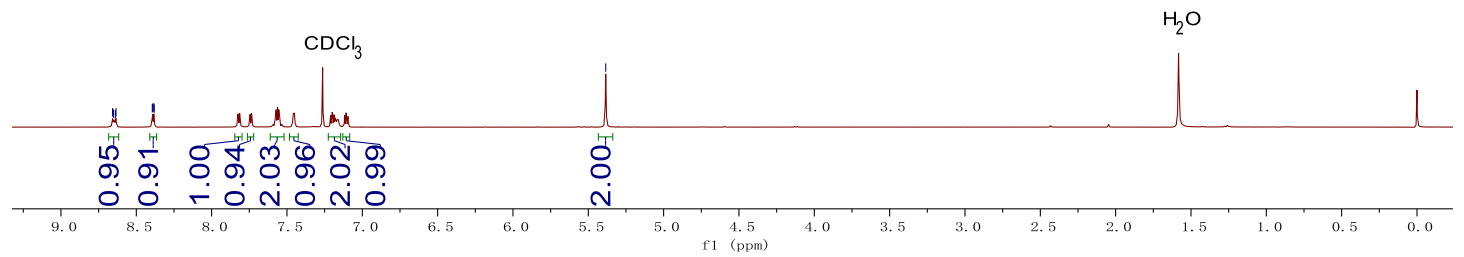

Compound $3 x^{1} \mathrm{H}$ NMR $400 \mathrm{MHz}$

ก๊

लं

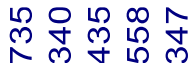

กొ

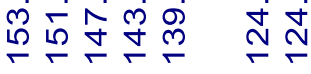

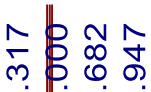

企电定

$\stackrel{\infty}{\sim} \stackrel{\infty}{\sim}$

-i, i, 位

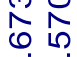

Nิ

$\hat{m} \stackrel{m}{\sim}$

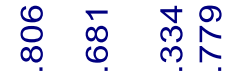

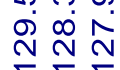

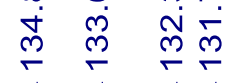

,

Imaxw

$\begin{array}{lllllllllllllll}138 & 137 & 136 & 135 & 134 & 133 & 132 & 131 & 130 & 129 & 128 & 127 & 126 & 125\end{array}$

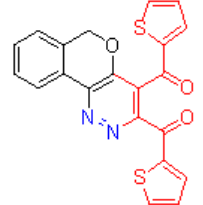

$3 \mathbf{x}$ in $\mathrm{CDC}_{3}$ 


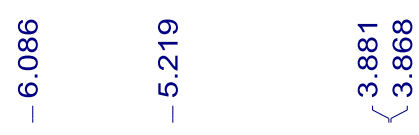
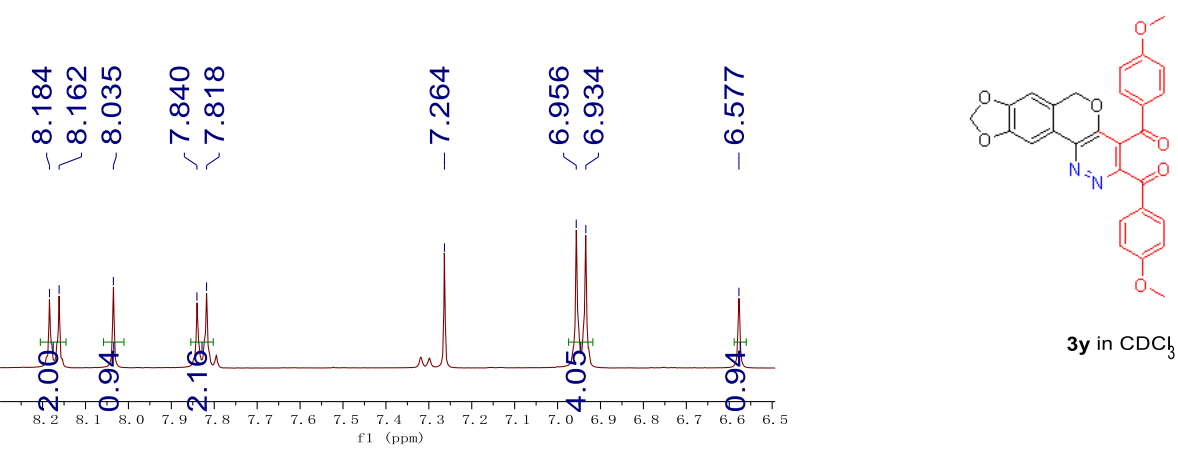

$3 y$ in $\mathrm{CDCb}$

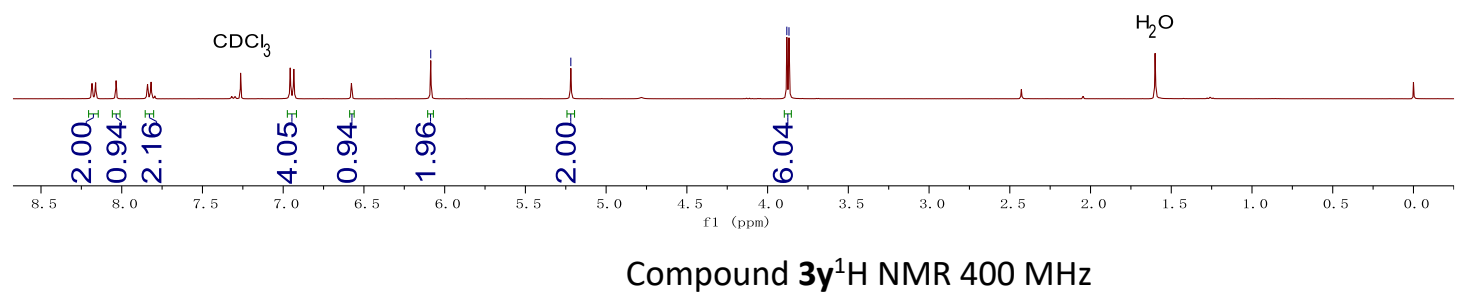

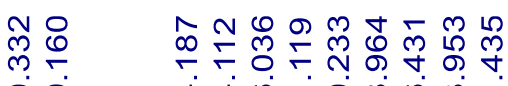

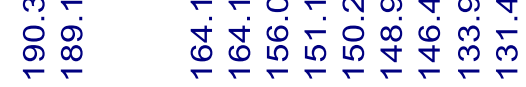

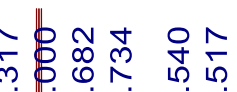

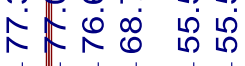

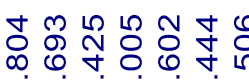

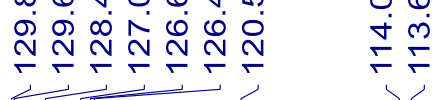

당요

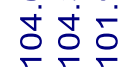

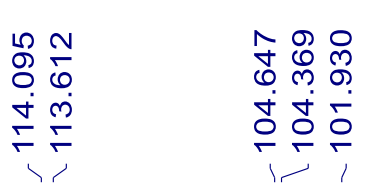
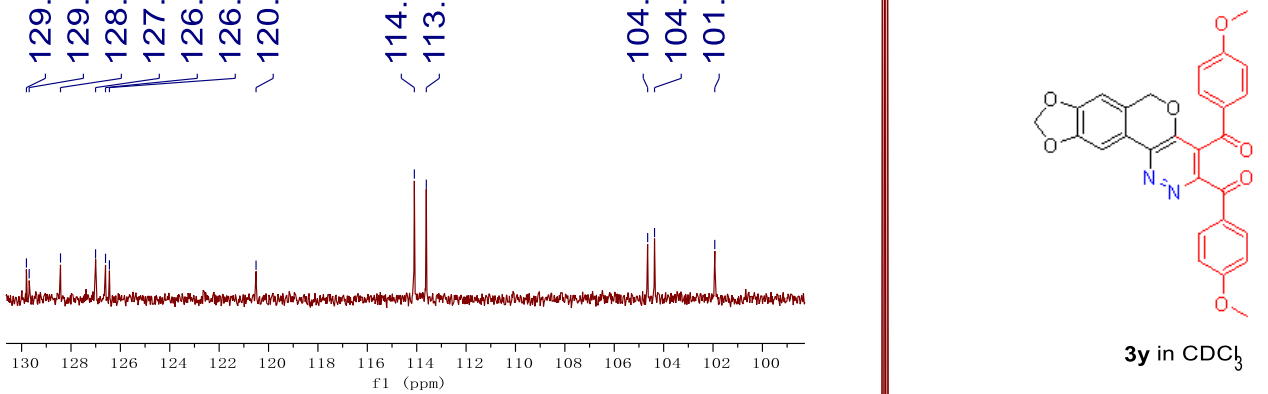

3y in $\mathrm{CDC}_{3}$
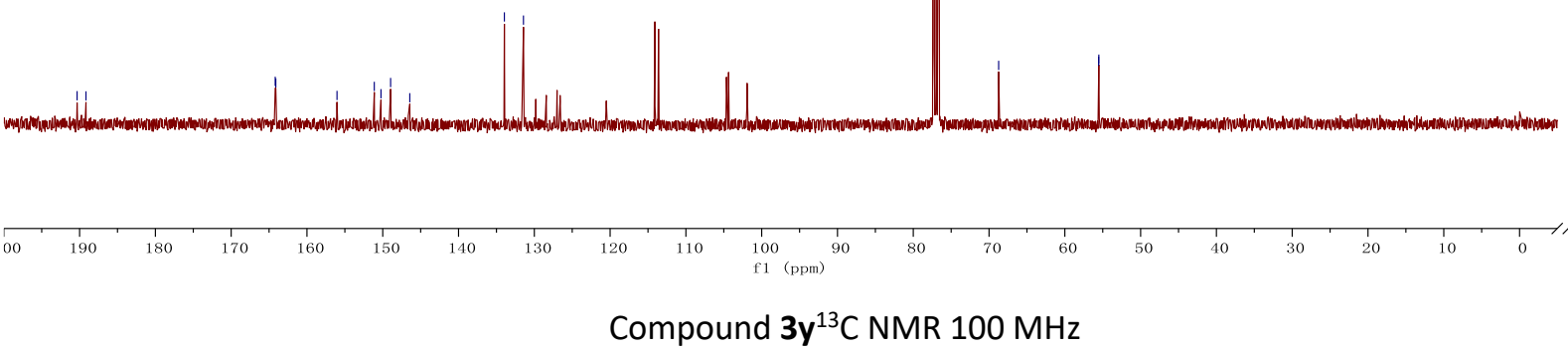

Compound $3 y^{13} \mathrm{C}$ NMR $100 \mathrm{MHz}$ 


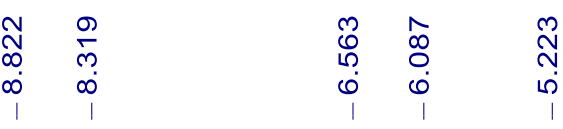

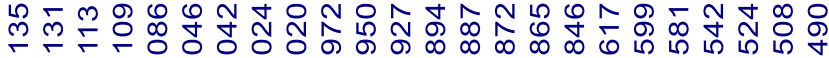

$\infty \infty_{\infty}^{\infty} \infty \infty_{\infty}^{\infty} \infty \infty^{\infty} \infty \infty^{\infty}$
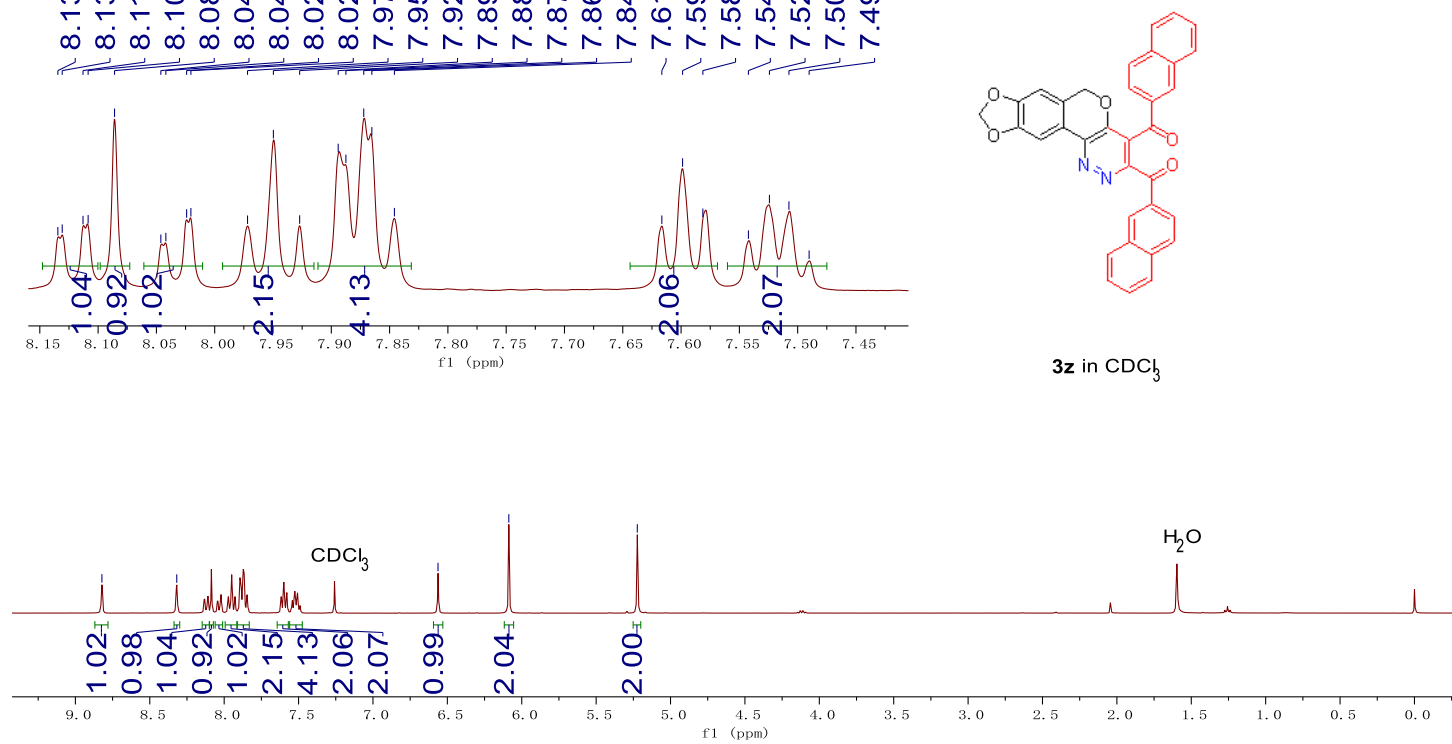

Compound $3 z^{1} \mathrm{H}$ NMR $400 \mathrm{MHz}$

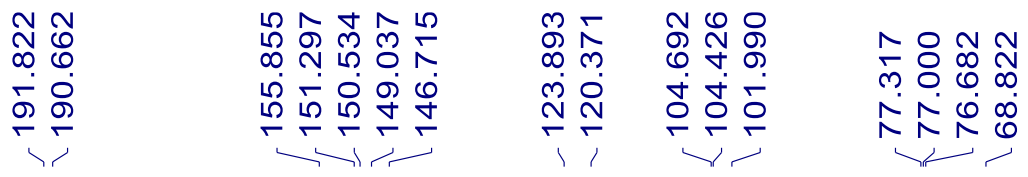

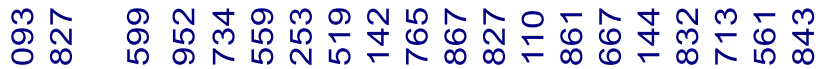

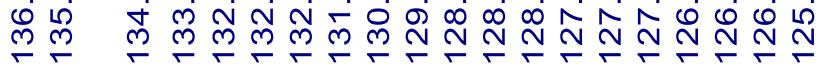
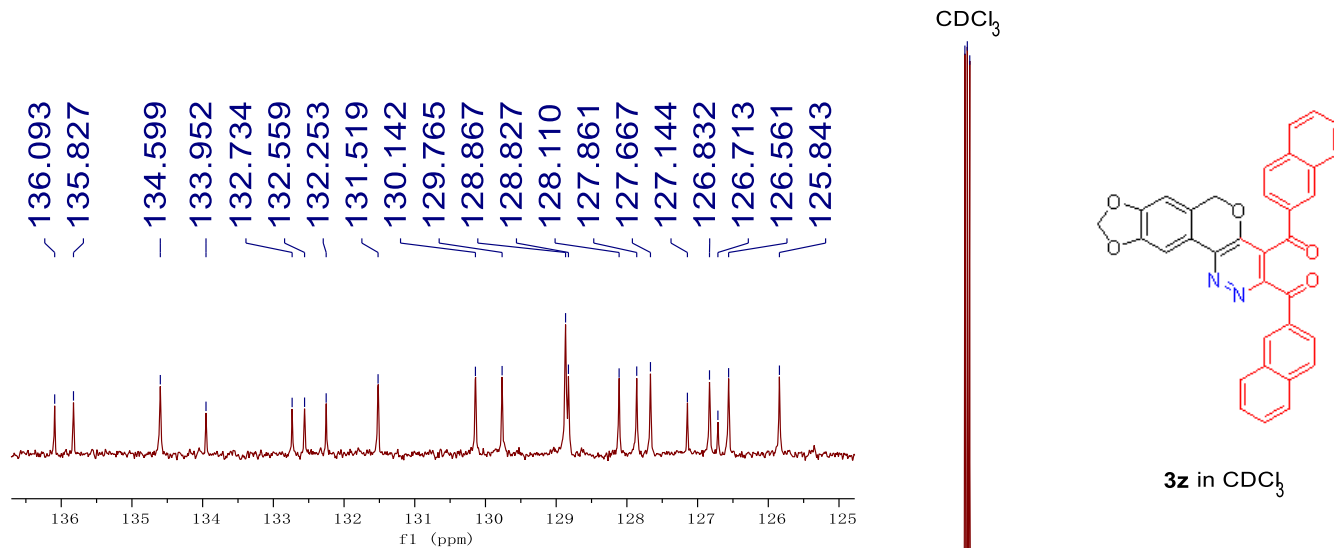

$3 \mathbf{z}$ in $\mathrm{CDCl}_{3}$

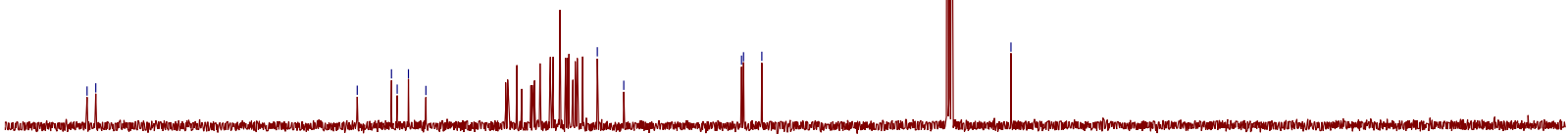

$\begin{array}{llllllll}1 & 1 & 1 & 1 & 1 & 1 & 1 & 1 \\ 200 & 190 & 180 & 170 & 160 & 150 & 140 & 130\end{array}$

Compound $3 z^{13} \mathrm{C}$ NMR $100 \mathrm{MHz}$ 


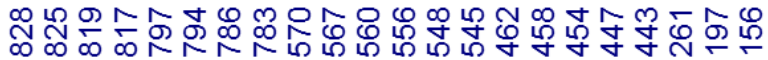

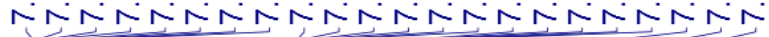
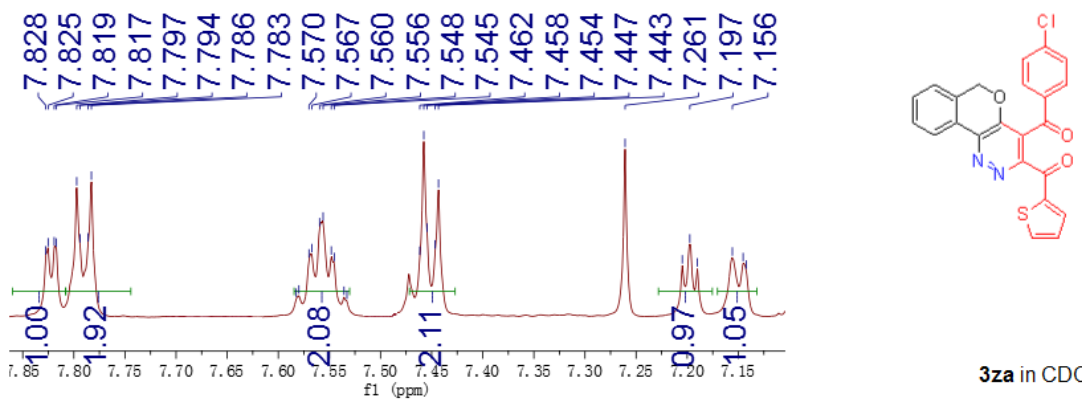

3za in $\mathrm{CDCl}_{3}$

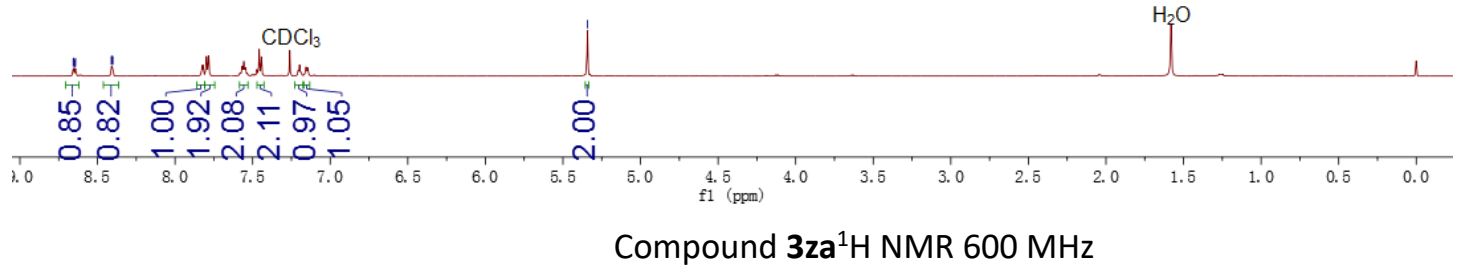

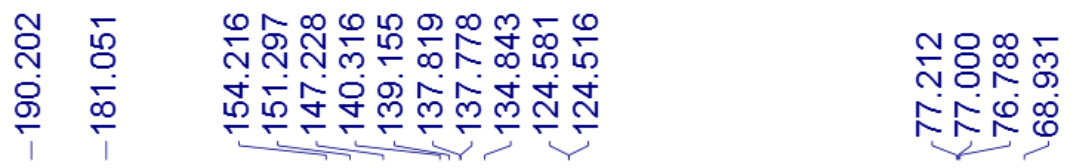

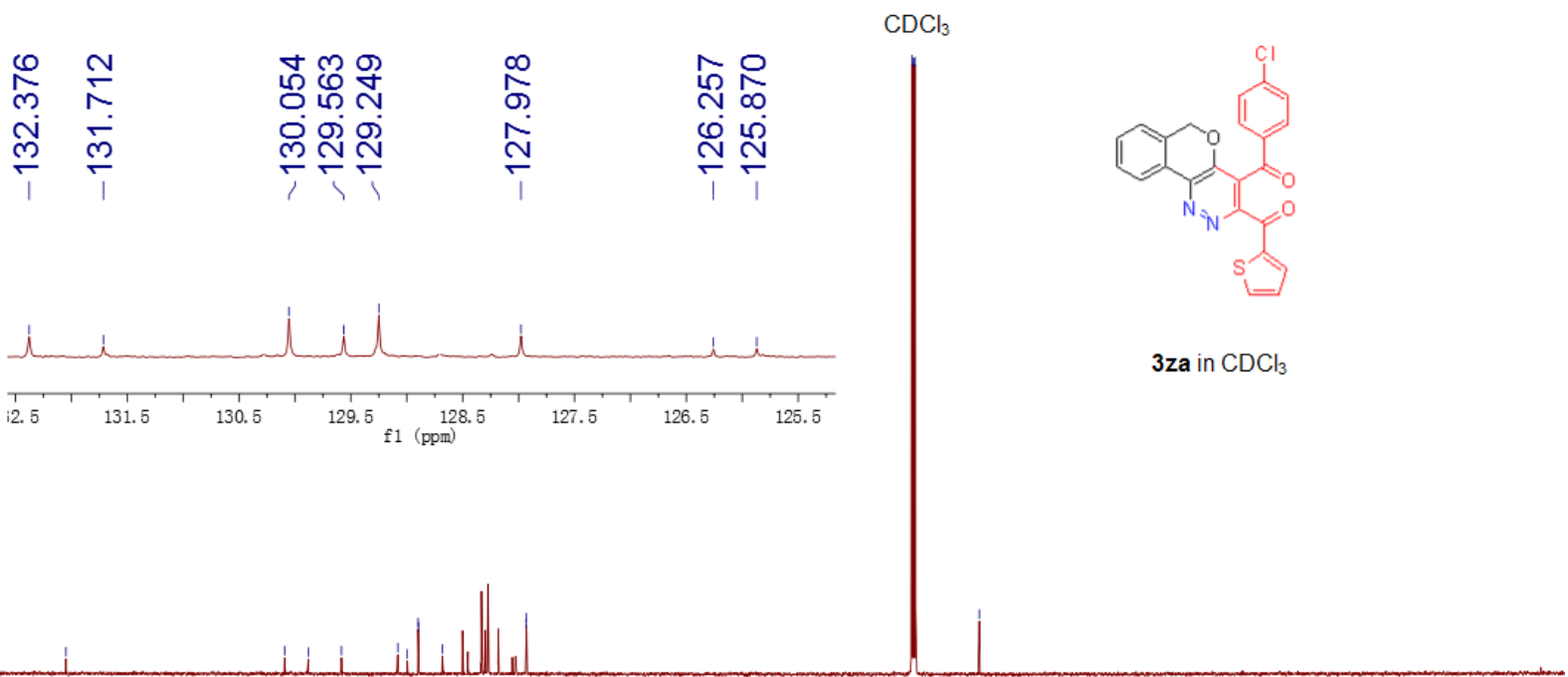

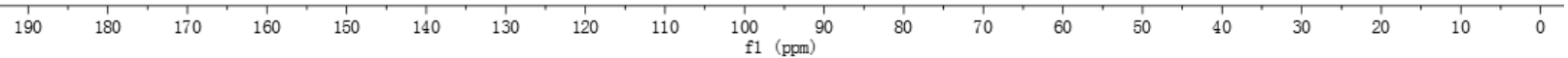
Compound $3 \mathrm{za}^{13} \mathrm{C}$ NMR $150 \mathrm{MHz}$ 


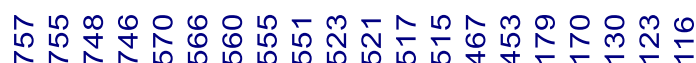

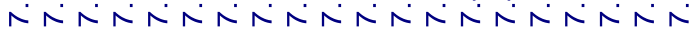
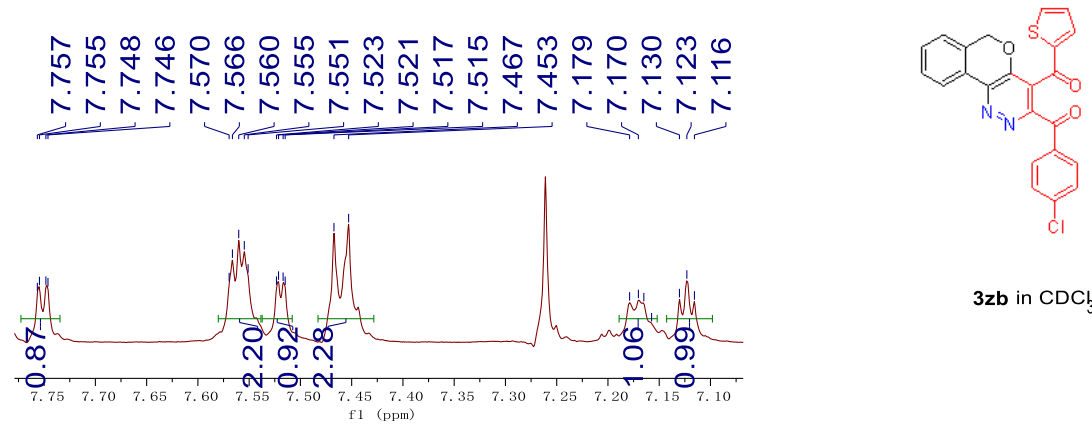

3zb in $\mathrm{CDCl}$ f1 (ppm)

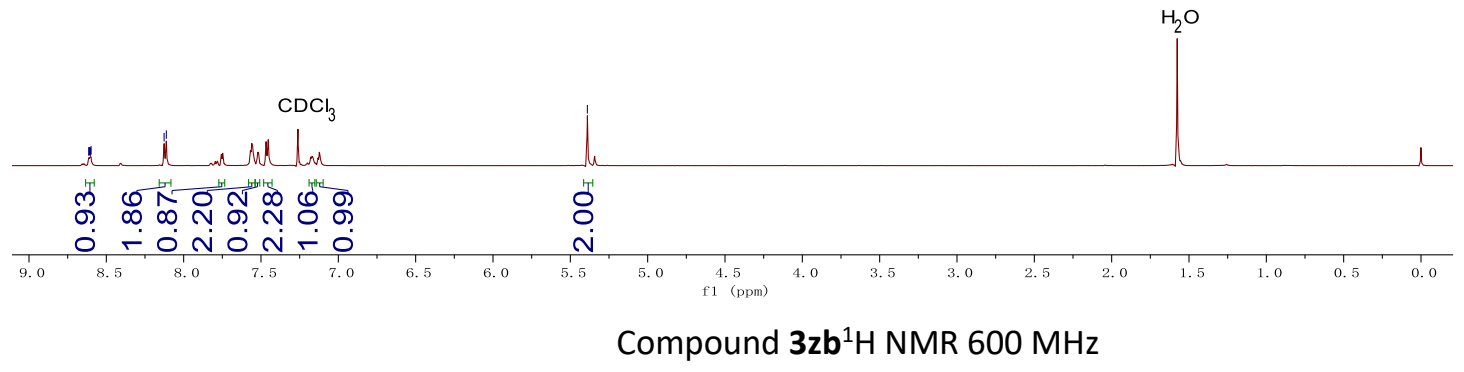

$\begin{array}{ll}\infty & 0 \\ 0 & 0 \\ 10 & 0 \\ \infty & \text { mं } \\ \infty & \infty \\ 1 & 1\end{array}$

\begin{tabular}{|c|}
\hline 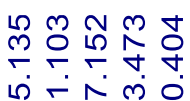 \\
\hline \\
\hline
\end{tabular}

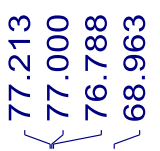

$\mathrm{CDCl}_{3}$
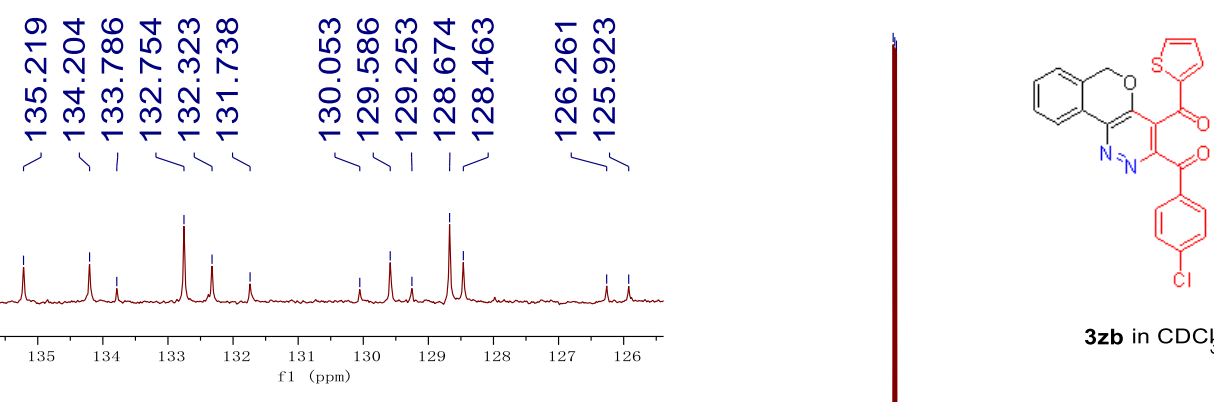

3zb in $\mathrm{CDC}$
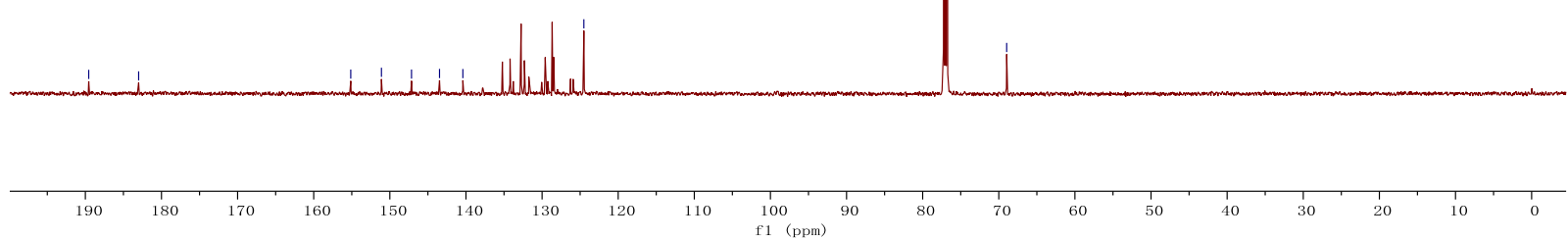

Compound 3zb ${ }^{13} \mathrm{C}$ NMR $150 \mathrm{MHz}$ 


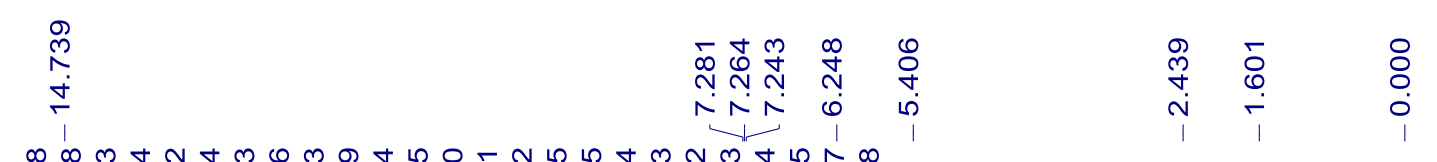

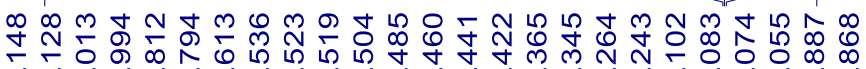

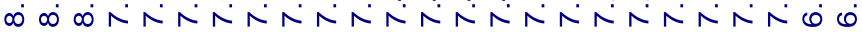
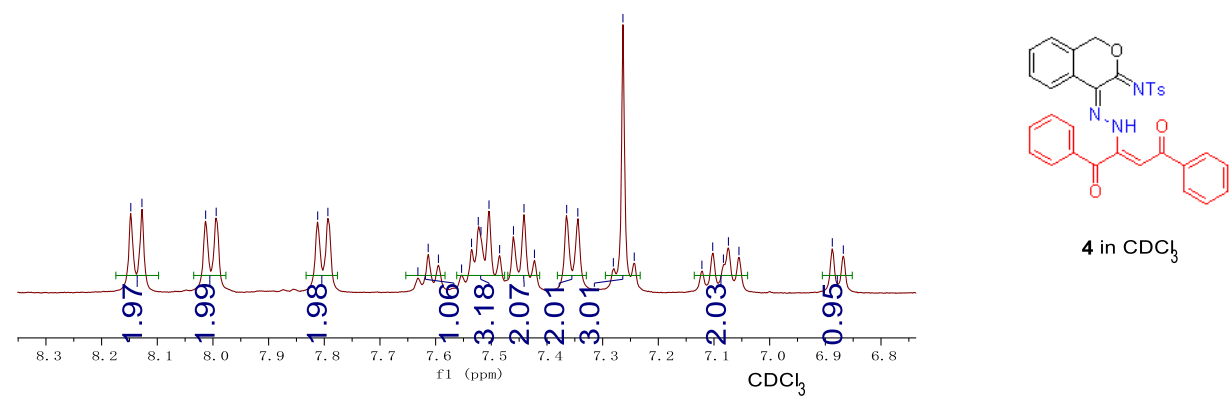

4 in $\mathrm{CDC}_{3}$

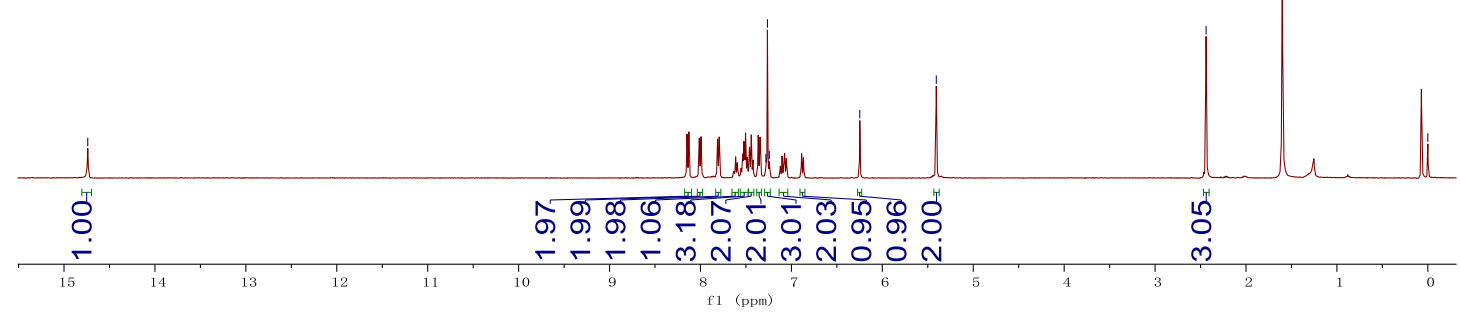

Compound $4{ }^{1} \mathrm{H}$ NMR $400 \mathrm{MHz}$

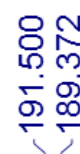

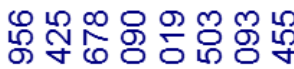

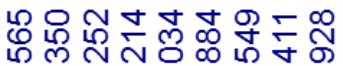

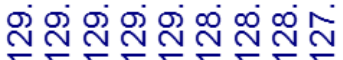

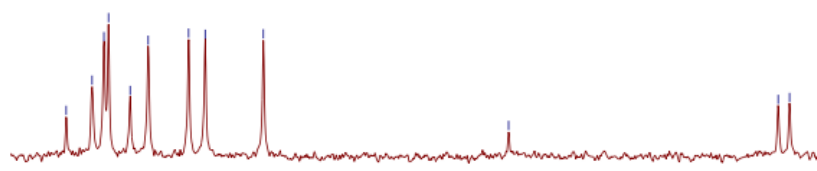
$\begin{array}{llllllllllllll}0.0 & 129.5 & 129.0 & 128.5 & 128.0 & 127.5 & 127.0 & 126.5 & 126.0 & 125.5 & 125.0 & 124.5 & 124.0 & 123.5\end{array}$

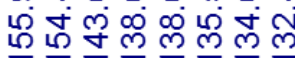

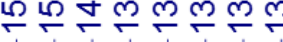

๙

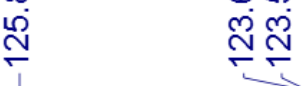

宁 穴

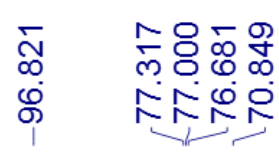

$\infty$
0
0

$\mathrm{CDCl}_{3}$

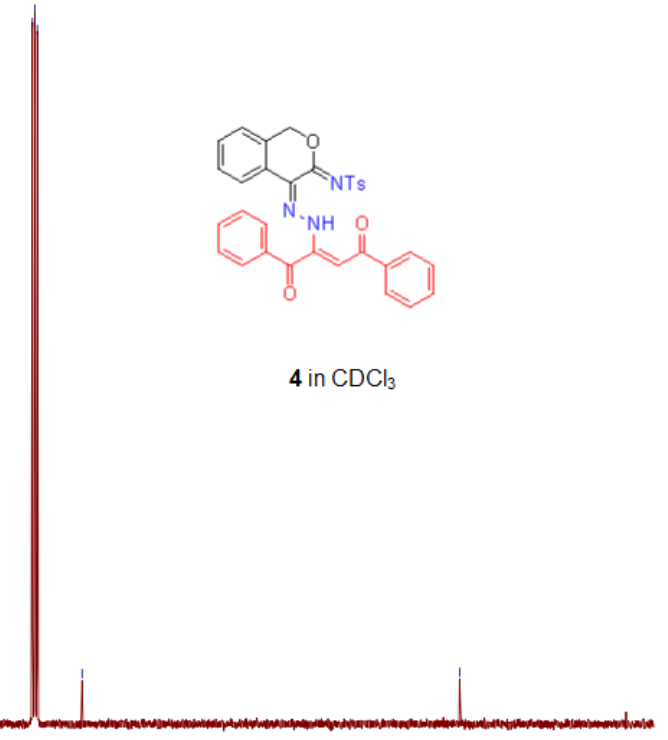

$\begin{array}{llllllll}190 & 180 & 170 & 160 & 150 & 140 & 130 & 120\end{array}$

\section{Compound4 ${ }^{13} \mathrm{C}$ NMR $100 \mathrm{MHz}$}

